\title{
Fierz-complete NJL model study. II. Toward the fixed-point and phase structure of hot and dense two-flavor QCD
}

\author{
Jens Braun, ${ }^{1,2, *}$ Marc Leonhardt, ${ }^{1, \dagger}$ and Martin Pospiech ${ }^{1, \$}$ \\ ${ }^{1}$ Institut für Kernphysik (Theoriezentrum), Technische Universität Darmstadt, D-64289 Darmstadt, Germany \\ ${ }^{2}$ ExtreMe Matter Institute EMMI, GSI, Planckstrasse 1, D-64291 Darmstadt, Germany
}

(Received 22 February 2018; published 23 April 2018)

\begin{abstract}
Nambu-Jona-Lasinio-type models are often employed as low-energy models for the theory of the strong interaction to analyze its phase structure at finite temperature and quark chemical potential. In particular, at low temperature and large chemical potential, where the application of fully first-principles approaches is currently difficult at best, this class of models still plays a prominent role in guiding our understanding of the dynamics of dense strong-interaction matter. In this work, we consider a Fierz-complete version of the Nambu-Jona-Lasinio model with two massless quark flavors and study its renormalization group flow and fixed-point structure at leading order of the derivative expansion of the effective action. Sum rules for the various four-quark couplings then allow us to monitor the strength of the breaking of the axial $U_{\mathrm{A}}(1)$ symmetry close to and above the phase boundary. We find that the dynamics in the ten-dimensional Fierzcomplete space of four-quark couplings can only be reduced to a one-dimensional space associated with the scalar-pseudoscalar coupling in the strict large- $N_{\mathrm{c}}$ limit. Still, the interacting fixed point associated with this one-dimensional subspace appears to govern the dynamics at small quark chemical potential even beyond the large- $N_{\mathrm{c}}$ limit. At large chemical potential, corrections beyond the large- $N_{\mathrm{c}}$ limit become important, and the dynamics is dominated by diquarks, favoring the formation of a chirally symmetric diquark condensate. In this regime, our study suggests that the phase boundary is shifted to higher temperatures when a Fierz-complete set of four-quark interactions is considered.
\end{abstract}

DOI: 10.1103/PhysRevD.97.076010

\section{INTRODUCTION}

Despite the tremendous progress that has been made in the development of fully first-principles approaches to the theory of the strong interaction in recent years, low-energy models of the theory of the strong interaction (QCD) are still considered very valuable for a variety of reasons. In particular, in the high-density regime, which is at least difficult to access with lattice Monte Carlo techniques, the Nambu-Jona-Lasinio (NJL) model and its various variations and relatives, such as quark-meson (QM) models, provide us with an insight into the rich symmetry breaking patterns that may potentially be at work in this regime. The interest in strong-interaction matter at densities beyond the nuclear saturation density is by no means academic but rather essential for our understanding of the dynamics of

\footnotetext{
*jens.braun@physik.tu-darmstadt.de leonhardt@theorie.ikp.physik.tu-darmstadt.de ¥martinp@theorie.ikp.physik.tu-darmstadt.de
}

Published by the American Physical Society under the terms of the Creative Commons Attribution 4.0 International license. Further distribution of this work must maintain attribution to the author(s) and the published article's title, journal citation, and DOI. Funded by SCOAP ${ }^{3}$. astrophysical objects. For example, studies of neutron stars rely on the equation of state of strong-interaction matter as input, which, however, is currently plagued by significant uncertainties at least at high density; see, e.g., Ref. [1] for a recent review.

From its early days on, the NJL model-originally introduced as an effective theory to describe spontaneous symmetry breaking in particle physics based on an analogy with superconducting materials [2] — has guided our understanding of the dynamics underlying the QCD phase structure; see Refs. [3-7] for reviews.

The great relevance of NJL-type model studies for our understanding of dense strong-interaction matter is undisputed. Still, it is also part of the truth that the corresponding predictions suffer from generic features of the NJL model as well as from approximations underlying these studies. First of all, it should be mentioned that NJL-type models in four space-time dimensions are nonrenormalizable, both on the perturbative as well as on the nonperturbative levels (see, e.g., Refs. [8,9]). Therefore, the UV cutoff scale becomes a parameter of the model, and as an immediate consequence, the regularization scheme belongs to the definition of the model. In particular, this implies that a given value of the UV cutoff scale has always to be viewed against the background of the chosen regularization 
scheme. From a renormalization group (RG) standpoint, this scale should anyhow not be considered as an actual UV extent of the model but rather as the scale at which the couplings of the model are fixed. In addition, we note that the use of so-called three-dimensional/spatial regularization schemes for studies of hot and dense matter may not be unproblematic. This issue originates from the fact that this class of schemes explicitly breaks Poincaré invariance, even at zero temperature where the model parameters are usually fixed. This may then eventually lead to spuriously emerging symmetry breaking patterns [10].

Let us now turn to more specific aspects of NJL-type model studies. The so-called classical action underlying the latter is usually given by a kinetic term for the quarks and a set of fourquark interactions which are usually selected by phenomenological reasoning. These interactions may be viewed as dynamically generated by quark-gluon interactions at highenergy scales. In fact, two-gluon exchange diagrams do not only generate immediately a scalar-pseudoscalar four-quark self-interaction channel as mostly considered in NJL-type models but all four-quark self-interaction channels compatible with the fundamental symmetries of QCD. From the standpoint of an RG evolution of QCD from high to low energies, the gluon-induced four-quark interactions may then become strong enough to trigger spontaneous symmetry breaking at some intermediate-energy scale, depending on, e.g., the temperature and the quark chemical potential. This scale may be associated with the UV cutoff scale of NJL-type models. In this spirit, we may therefore consider the general form of NJL-type models to be rooted in QCD. In practice, however, the four-quark couplings in NJL-type models are usually not fixed in this way but rather by tuning them such that the correct values of a given set of low-energy observables is reproduced at, e.g., vanishing temperature and quark chemical potential. Unfortunately, the values of the chosen set of low-energy observables may in general be reproduced by various different sets of parameters. Moreover, the parameters may depend on, e.g., the temperature and the quark chemical potential as suggested by the aforementioned RG evolution of gluon-induced four-quark interactions [11].

From a phenomenological point of view, the four-quark interaction channels may be recast into effective bosonic degrees of freedom (d.o.f.). In particular, at large chemical potential, the effective d.o.f. associated with the scalarpseudoscalar channel, namely, the $\sigma$ meson and the pions, are no longer expected to dominate the low-energy physics. Here, other d.o.f., such as diquarks, play a dominant role; see, e.g., Refs. [5,12-14] for reviews. Apart from this phenomenologically guided point of view, the inclusion of more than one four-quark channel is of field-theoretical relevance as a given pointlike four-quark interaction channel is reducible by means of so-called Fierz transformations. This naturally causes ambiguities in studies in which the considered set of four-quark interaction channels is incomplete and also implies the necessity to use very general Ansätze for the quark propagator as employed in, e.g., Dyson-Schwinger-type studies [15]. QCD low-energy model studies are in general treated in approximations which are indeed incomplete with respect to Fierz transformations. Note also that, even in studies of the conventional NJL/QM model defined with only a scalar-pseudoscalar four-quark interaction channel, other four-quark channels are dynamically generated due to quantum fluctuations.

By considering an NJL model with a single fermion species in Ref. [10], we have demonstrated that Fierz completeness as associated with the inclusion of more "exotic" four-fermion channels does not only play a prominent role at large chemical potential but also affects the dynamics at small chemical potential. The latter is illustrated by a significant dependence of the curvature of the finite-temperature phase boundary at small chemical potential on the number of four-fermion channels included in the calculations. In the present work, which is a sequel of Ref. [10], we now extend our analysis to an NJL model with massless quark flavors coming in $N_{\mathrm{c}}$ colors and two flavors to gain a better understanding of how Fierzincomplete approximations of QCD low-energy models affect the predictions for the phase structure at finite temperature and density. In particular, we take into account the explicit symmetry breaking arising from the presence of a heat bath and the chemical potential in our present study anchored at the leading order of the derivative expansion of the effective action. Within our Fierz-complete framework including ten four-quark channels, we observe that channels associated with an explicit breaking of Poincaré invariance tend to increase significantly the critical temperature at large chemical potential. In accordance with many conventional model studies (see, e.g., Refs. [5,6,13,14] for reviews), diquarks are nevertheless found to be the most dominant d.o.f. in this regime.

In Sec. II, we discuss our model and aspects of symmetries which are relevant for our present analysis. Whereas the number of colors $N_{\mathrm{c}}$ is a free parameter in this more general discussion, we emphasize that we exclusively consider $N_{\mathrm{c}}=3$ in all numerical studies in this work. Our RG framework is introduced in Sec. III. However, since this work is a sequel of Ref. [10], in which also the theoretical setting underlying our present analysis has been thoroughly discussed, we refrain here from a presentation of details regarding our RG framework. The RG fixed-point and phase structure of our model at finite temperature and density at leading order of the derivative expansion of the effective action is analyzed in Sec. IV, in which we begin with a discussion of the relation of the mean-field approximation and an RG study of four-quark interactions in a one-channel approximation in Sec. IV A. The actual analysis of the fixedpoint and phase structure of our Fierz-complete NJL-type model can be found in Secs. IV B, IV C, IV D, IV E. The symmetry breaking patterns are discussed in Sec. IV B. The effect of $U_{\mathrm{A}}(1)$ breaking and its fate at high temperature is analyzed in Sec. IV C. To gain a better understanding of the 
mechanisms underlying symmetry breaking at finite temperature and density in our Fierz-complete study involving ten four-quark interaction channels, we then discuss the RG flow of the Fierz-complete system in the large- $N_{\mathrm{c}}$ limit in Sec. IV D. In Sec. IV E, we finally analyze the dynamics of the Fierz-complete system with the aid of a two-channel approximation, which is composed of the aforementioned scalar-pseudoscalar channel and a diquark channel often employed in conventional NJL model studies. There, we also comment on the effect of Fierz-incomplete approximations on the curvature of the finite-temperature phase boundary at small chemical potential. Our conclusions can be found in Sec. V.

\section{MODEL}

A frequently employed approximation in terms of NJLtype models is to consider an action which only consists of a kinetic term for the quarks and a scalar-pseudoscalar four-quark interaction channel. By means of a HubbardStratonovich transformation, auxiliary fields can be introduced, and the four-quark interaction channel is converted into a (screening) mass term for the auxiliary fields and a Yukawa interaction channel between the latter and the quarks. Conventionally, the auxiliary fields are chosen to carry the quantum numbers of the $\sigma$ meson and the pions in case of the scalar-pseudoscalar four-quark interaction channel. The interactions between the quarks are then said to be mediated by an exchange of the aforementioned mesons. For a study of chiral symmetry breaking, this choice for the auxiliary fields is particularly convenient because it allows for a straightforward projection on the chiral order parameter. In this work, however, we shall consider a purely fermionic formulation of the NJL model with massless quarks coming in two flavors and $N_{\mathrm{c}}$ colors, aiming at an analysis of the effect of Fierz-incomplete approximations on the phase structure at finite temperature and density in QCD low-energy model studies. To relate our work to the latter studies, we start our discussion by considering a so-called classical action $S$, which essentially consists of a kinetic term for the quarks and a scalarpseudoscalar four-quark interaction channel in four Euclidean space-time dimensions:

$$
\begin{aligned}
S[\bar{\psi}, \psi]= & \int_{0}^{\beta} \mathrm{d} \tau \int \mathrm{d}^{3} x\left\{\bar{\psi}\left(\mathrm{i} \not \partial-\mathrm{i} \mu \gamma_{0}\right) \psi\right. \\
& \left.+\frac{1}{2} \bar{\lambda}_{(\sigma-\pi)}\left[(\bar{\psi} \psi)^{2}-\left(\bar{\psi} \gamma_{5} \tau_{i} \psi\right)^{2}\right]\right\} .
\end{aligned}
$$

Here, $\beta=1 / T$ is the inverse temperature, $\mu$ is the quark chemical potential, and $\bar{\lambda}_{(\sigma-\pi)}$ is the coupling associated with the scalar-pseudoscalar channel. The $\tau_{i}$ 's represent the Pauli matrices and couple the spinors in flavor space. Note that the quark fields $\psi$ carry Dirac, color, and flavor indices. In the following, they are assumed to be contracted pairwise, e.g., $(\bar{\psi} \mathcal{O} \psi) \equiv \bar{\psi}_{\chi} \mathcal{O}_{\chi_{\xi}^{\xi}} \psi_{\xi}$, where $\xi$ and $\chi$ represent collective indices for the Dirac, flavor, and color indices and $\mathcal{O}$ represents an operator specifying the properties of the interaction channel. Suitable insertions of 1-operators in Dirac, color, and flavor space are tacitly assumed.

Let us begin our symmetry analysis by noting that the action (1) is invariant under (global) $S U\left(N_{\mathrm{c}}\right)$ color rotations of the quark fields. As we do not allow for an explicit quark mass term, we also have an invariance under (independent) global flavor rotations of the left- and right-handed quark fields, $\psi_{\mathrm{L}, \mathrm{R}}=\frac{1}{2}\left(1 \pm \gamma_{5}\right) \psi$; i.e., the action is invariant under $S U_{\mathrm{L}}(2) \otimes S U_{\mathrm{R}}(2) \quad$ transformations. The spontaneous breakdown of this so-called chiral symmetry is associated with the formation of a corresponding chiral condensate $\langle\bar{\psi} \psi\rangle$ rendering the quarks massive.

The action (1) is also invariant under simple global phase transformations,

$$
U_{\mathrm{V}}(1): \bar{\psi} \mapsto \bar{\psi} \mathrm{e}^{-\mathrm{i} \alpha}, \quad \psi \mapsto \mathrm{e}^{\mathrm{i} \alpha} \psi,
$$

but is not invariant under axial phase transformations:

$$
U_{\mathrm{A}}(1): \bar{\psi} \mapsto \bar{\psi} \mathrm{e}^{\mathrm{i} \gamma_{5} \alpha}, \quad \psi \mapsto \mathrm{e}^{\mathrm{i} \gamma_{5} \alpha} \psi .
$$

In both cases, $\alpha$ denotes the "rotation" angle. Note that, in contrast to the case of a single fermion species, i.e., the case of one color and one flavor, a broken $U_{\mathrm{A}}(1)$ symmetry does not necessarily entail the existence of a finite expectation value $\langle\bar{\psi} \psi\rangle$ as associated with spontaneous chiral symmetry breaking. However, the spontaneous breakdown of the chiral symmetry also entails the breakdown of the $U_{\mathrm{A}}(1)$ symmetry [16]. In nature, it turns out that the $U_{\mathrm{A}}(1)$ symmetry is not realized but anomalously broken by topologically nontrivial gauge configurations [17,18], even if the chiral $S U_{\mathrm{L}}(2) \otimes S U_{\mathrm{R}}(2)$ is restored. For the phenomenologically more relevant three-flavor case, the explicit breaking of the $U_{\mathrm{A}}(1)$ symmetry has been put forward even earlier to explain the $\eta-\eta^{\prime}$ mixing in the mesonic mass spectrum [19]. The absence of the $U_{\mathrm{A}}(1)$ symmetry may be deduced from other observations as well. For example, we do not observe parity doubling of hadronic states [20], at least at low energies. In any case, in the action (1), we can artificially restore the $U_{\mathrm{A}}(1)$ symmetry by adding an additional four-quark channel,

$$
\sim \operatorname{det}\left(\bar{\psi}\left(1+\gamma_{5}\right) \psi\right)+\operatorname{det}\left(\bar{\psi}\left(1-\gamma_{5}\right) \psi\right),
$$

provided that the coupling associated with this channel is adjusted suitably relative to the coupling $\bar{\lambda}_{(\sigma-\pi)}$ of the scalarpseudoscalar channel; see also Ref. [4]. ${ }^{1}$ Indeed, the topologically nontrivial gauge configurations violating the $U_{\mathrm{A}}(1)$ symmetry can be recast into a four-quark interaction channel of the form (4) in the case of two-flavor QCD

\footnotetext{
${ }^{1}$ Note that the determinant in Eq. (4) is taken in flavor space.
} 
[18,21-24]. We shall come back to the issue of $U_{\mathrm{A}}(1)$ symmetry breaking below.

Apart from the chiral symmetry and the $U_{\mathrm{A}}(1)$ symmetry, the $U_{\mathrm{V}}(1)$ symmetry associated with baryon number conservation may also be spontaneously broken. In contrast to chiral symmetry breaking, this is indicated by the formation of, e.g., a diquark condensate $\delta^{a}$ carrying a net baryon and net color charge:

$$
\delta^{a} \sim\left\langle\mathrm{i} \bar{\psi}^{C} \gamma_{5} \epsilon_{f} \varepsilon_{c}^{l} \psi\right\rangle .
$$

Here, $\epsilon_{f} \equiv \epsilon_{f}^{(\alpha, \beta)}$ and $\varepsilon_{c}^{l} \equiv \varepsilon_{c}^{l(m, n)}$ are antisymmetric tensors in flavor and color space, respectively. Moreover, we have introduced charge-conjugated fields $\psi^{C}=\mathcal{C} \bar{\psi}^{T}$ and $\bar{\psi}^{C}=$ $\psi^{T} \mathcal{C}$ with $\mathcal{C}=\mathrm{i} \gamma_{2} \gamma_{0}$ being related to the charge conjugation operator.

The diquark condensate $\delta^{a}$ is a state with $J^{P}=0^{+}$and has been found to be most dominantly generated by onegluon exchange [25] and topologically nontrivial gauge configurations [25,26]; see also Refs. [13,23,27] for reviews. The flavor antisymmetric structure of this colorsuperconducting condensate corresponds to a singlet representation of the global chiral group, which implies that the formation of such a condensate does not violate the chiral symmetry of the theory [28]. Note that this is different in QED-like theories in which the formation of a superconducting ground state also requires the chiral symmetry to be broken; see, e.g., Ref. [10]. Instead, the formation of the aforementioned color-superconducting condensate $\delta^{a}$ in QCD comes at the price of a broken $S U\left(N_{\mathrm{c}}\right)$ color symmetry.

In addition to the breaking of the aforementioned symmetries, we have to deal with the explicit breaking of Poincaré invariance in our calculations because of the presence of a heat bath and a finite quark chemical potential. This implies that the Euclidean time direction is distinguished and we are only left with rotational invariance among the spatial components. With respect to the fundamental symmetries associated with charge conjugation, time reversal, and parity, we add that only invariance under parity transformations and time reversal transformations remain intact in the presence of a finite quark chemical potential as the latter also breaks explicitly the charge conjugation symmetry.

Let us now discuss the general form of the quantum effective action $\Gamma$ of our model at leading order of the derivative expansion. Our symmetry considerations constrain the classical action of the model underlying our studies. The latter action may be considered as the zeroth-order approximation of the corresponding quantum effective action $\Gamma$. The full quantum effective action is obtained from the path integral associated with the classical action by means of a Legendre transformation. By computing the quantum corrections to the classical action (1), we immediately observe that four-quark channels other than the scalar-pseudoscalar channel are generated. In fact, any operator respecting the symmetries of our model can in principle be generated. Structuring our calculations by means of a derivative expansion, we find that, at leading order (LO), the most general Ansatz for the effective average action compatible with the symmetries of the theory reads ${ }^{2}$

$$
\begin{aligned}
\Gamma_{\mathrm{LO}}[\bar{\psi}, \psi]= & \int_{0}^{\beta} \mathrm{d} \tau \int \mathrm{d}^{3} x\left\{\bar{\psi}\left(Z^{\|} \mathrm{i} \gamma_{0} \partial_{0}+Z^{\perp} \mathrm{i} \gamma_{i} \partial_{i}-Z_{\mu} \mathrm{i} \mu \gamma_{0}\right) \psi\right. \\
& \left.+\frac{1}{2} \sum_{j \in \mathcal{B}} Z_{j} \bar{\lambda}_{j} \mathcal{L}_{j}\right\}
\end{aligned}
$$

where the elements $\mathcal{L}_{j}$ form a ten-component Fierzcomplete basis $\mathcal{B}$ of pointlike four-quark interactions accompanied by the associated bare couplings $\bar{\lambda}_{i}$ and the corresponding vertex renormalizations $Z_{j}{ }^{3}$ Any other pointlike four-quark interaction invariant under the symmetries of our model is indeed reducible by means of Fierz transformations. ${ }^{4}$ Recall that we assume here that the $U_{\mathrm{A}}(1)$ symmetry is broken explicitly; see below for a detailed discussion of this issue. The renormalization factors associated with the kinetic term are given by $Z^{\|}$and $Z^{\perp}$, respectively. In the following, we set $Z^{\|}=Z^{\perp} \equiv 1$ as the $R G$ flow of these quantities vanishes identically at leading order of the derivative expansion; see Ref. [8]. In general, the chemical potential is also accompanied by a renormalization factor $Z_{\mu}$. As a consequence of the so-called Silver-Blaze property of general quantum field theories [29-31], however, we have $Z_{\mu}^{-1}=Z^{\|}=Z^{\perp}$ at $T=0$, provided that the renormalized chemical potential $\mu_{\mathrm{r}} \equiv Z_{\mu} \mu$ is smaller than the dynamically generated renormalized (pole) mass $\bar{m}_{\mathrm{q}}=$ $\bar{m}_{\mathrm{q}} / Z^{\perp}$ of the quarks [10].

Now, we specify the Fierz-complete basis of pointlike four-quark interactions, which we use to parametrize the effective action (6). As indicated above, we find that this basis is composed of ten four-quark channels. We choose six of them to be invariant under $S U\left(N_{\mathrm{c}}\right) \otimes S U_{\mathrm{L}}(2) \otimes$ $S U_{\mathrm{R}}(2) \otimes U_{\mathrm{V}}(1) \otimes U_{\mathrm{A}}(1)$ transformations:

$$
\mathcal{L}_{(V+A)_{\|}}=\left(\bar{\psi} \gamma_{0} \psi\right)^{2}+\left(\bar{\psi} \mathrm{i} \gamma_{0} \gamma_{5} \psi\right)^{2}
$$

\footnotetext{
${ }^{2}$ Quark self-interactions of higher order are also generated dynamically, but at leading order of the derivative expansion, they do not contribute to the RG flow of the four-quark selfinteractions and are therefore not included in our present study.

${ }^{3}$ The leading order of the derivative expansion implies that the four-quark self-interactions are treated in the pointlike limit.

${ }^{4}$ The couplings $\bar{\lambda}_{j}$ appearing in the effective action (6) should not be confused with the couplings $\bar{\lambda}_{j}$ appearing in the classical action $S$; see, e.g., Eq. (1). The couplings appearing in the effective action include quantum corrections, whereas, from an RG standpoint, the couplings appearing in the classical action only determine the values of the RG flows of the four-quark couplings at the initial scale $\Lambda$.
} 


$$
\begin{gathered}
\mathcal{L}_{(V+A)_{\perp}}=\left(\bar{\psi} \gamma_{i} \psi\right)^{2}+\left(\bar{\psi} \mathrm{i} \gamma_{i} \gamma_{5} \psi\right)^{2}, \\
\mathcal{L}_{(V-A)_{\|}}=\left(\bar{\psi} \gamma_{0} \psi\right)^{2}-\left(\bar{\psi} \mathrm{i} \gamma_{0} \gamma_{5} \psi\right)^{2}, \\
\mathcal{L}_{(V-A)_{\perp}}=\left(\bar{\psi} \gamma_{i} \psi\right)^{2}-\left(\bar{\psi} \mathrm{i} \gamma_{i} \gamma_{5} \psi\right)^{2}, \\
\mathcal{L}_{(V+A)_{\|}^{\text {adj }}}=\left(\bar{\psi} \gamma_{0} T^{a} \psi\right)^{2}+\left(\bar{\psi} \mathrm{i} \gamma_{0} \gamma_{5} T^{a} \psi\right)^{2}, \\
\mathcal{L}_{(V-A)_{\perp}^{\mathrm{adj}}}=\left(\bar{\psi} \gamma_{i} T^{a} \psi\right)^{2}-\left(\bar{\psi} \mathrm{i} \gamma_{i} \gamma_{5} T^{a} \psi\right)^{2} .
\end{gathered}
$$

The remaining four channels are then chosen to be invariant under $S U\left(N_{\mathrm{c}}\right) \otimes S U_{\mathrm{L}}(2) \otimes S U_{\mathrm{R}}(2) \otimes U_{\mathrm{V}}(1)$ transformations but break the $U_{\mathrm{A}}(1)$ symmetry explicitly,

$$
\begin{gathered}
\mathcal{L}_{(\sigma-\pi)}=(\bar{\psi} \psi)^{2}-\left(\bar{\psi} \gamma_{5} \tau_{i} \psi\right)^{2}, \\
\mathcal{L}_{(S+P)_{-}=}(\bar{\psi} \psi)^{2}-\left(\bar{\psi} \gamma_{5} \tau_{i} \psi\right)^{2}+\left(\bar{\psi} \gamma_{5} \psi\right)^{2}-\left(\bar{\psi} \tau_{i} \psi\right)^{2}, \\
\mathcal{L}_{\mathrm{csc}}=4\left(\mathrm{i} \bar{\psi} \gamma_{5} \tau_{2} T^{A} \psi{ }^{C}\right)\left(\mathrm{i} \bar{\psi}{ }^{C} \gamma_{5} \tau_{2} T^{A} \psi\right), \\
\mathcal{L}_{(S+P)_{-}^{\text {adj }}}=\left(\bar{\psi} T^{a} \psi\right)^{2}-\left(\bar{\psi} \gamma_{5} \tau_{i} T^{a} \psi\right)^{2} \\
+\left(\bar{\psi} \gamma_{5} T^{a} \psi\right)^{2}-\left(\bar{\psi} \tau_{i} T^{a} \psi\right)^{2},
\end{gathered}
$$

where, e.g., $\left(\bar{\psi} \gamma_{5} \tau_{i} \psi\right)^{2} \equiv\left(\bar{\psi} \gamma_{5} \tau_{i} \psi\right)\left(\bar{\psi} \gamma_{5} \tau_{i} \psi\right)$ and the $T^{a}$ 's denote the generators of $S U\left(N_{\mathrm{c}}\right)$. Note that this basis is not unique. In principle, we can combine elements of the basis to perform a basis transformation. Our present choice is motivated by the four-quark channels conventionally employed in QCD low-energy models. Apparently, the scalar-pseudoscalar channel appearing in Eq. (1) is given by the channel $\mathcal{L}_{(\sigma-\pi)}$. A channel of the form of Eq. (4) is associated with the presence of topologically nontrivial gauge configurations and is given by the channel $\mathcal{L}_{(S+P)_{-}}$ up to a numerical constant. There is also a channel associated with the formation of a diquark condensate of the type (5) in our basis. In fact, taking into account that such a condensate leaves the chiral symmetry intact, the corresponding four-quark channel $\mathcal{L}_{\mathrm{csc}}$ can be constructed from the tensor structure of the condensate (5). Our conventions in Eq. (15) are such that we only sum over the antisymmetric $(A)$ generators of the $S U\left(N_{\mathrm{c}}\right)$ color group. The normalization of this channel is chosen as in the standard literature (see, e.g., Ref. [5]). Note that the channel $\mathcal{L}_{\mathrm{csc}}$ is invariant under $S U\left(N_{\mathrm{c}}\right) \otimes S U_{\mathrm{L}}(2) \otimes$ $S U_{\mathrm{R}}(2) \otimes U_{\mathrm{V}}(1)$ transformations. The formation of a diquark condensate then goes along with the breakdown of the $U_{\mathrm{V}}(1)$ symmetry as well as the $S U\left(N_{\mathrm{c}}\right)$ color symmetry. Finally, we add that the channel (16) may be viewed as a counterpart of the channel $\mathcal{L}_{(S+P)_{-}}$with a nontrivial color structure.

It is worth pointing out that our Fierz-complete set of pointlike four-quark interactions allows us to monitor $U_{\mathrm{A}}(1)$ symmetry breaking. Indeed, by requiring that the effective action $\Gamma$ is invariant under $U_{\mathrm{A}}(1)$ transformations, we find the following two sum rules for the four pointlike couplings violating the $U_{\mathrm{A}}(1)$ symmetry:

$$
\begin{gathered}
\mathcal{S}_{U_{\mathrm{A}}(1)}^{(1)}=\bar{\lambda}_{\mathrm{csc}}+\bar{\lambda}_{(S+P)_{-}^{\mathrm{adj}}}=0, \\
\mathcal{S}_{U_{\mathrm{A}}(1)}^{(2)}=\bar{\lambda}_{(S+P)_{-}}-\frac{N_{\mathrm{c}}-1}{2 N_{\mathrm{c}}} \bar{\lambda}_{\mathrm{csc}}+\frac{1}{2} \bar{\lambda}_{(\sigma-\pi)}=0 .
\end{gathered}
$$

These two sum rules are only fulfilled simultaneously if the $U_{\mathrm{A}}(1)$ symmetry of the theory is intact. For example, choosing only the scalar-pseudoscalar coupling $\bar{\lambda}_{(\sigma-\pi)}$ to be finite in the classical action (1), we find that the $U_{\mathrm{A}}(1)$ symmetry is violated. This symmetry is only found to be approximately restored on the quantum level at high temperatures; see our discussion in Sec. IV C.

From the sum rules (17) and (18), we deduce that the four-dimensional space spanned by the $U_{\mathrm{A}}(1)$-violating channels contains a $U_{\mathrm{A}}(1)$-symmetric subspace. In particular, the two sum rules imply that a Fierz-complete basis of pointlike four-quark interactions in case of a theory invariant under $S U\left(N_{\mathrm{c}}\right) \otimes S U_{\mathrm{L}}(2) \otimes S U_{\mathrm{R}}(2) \otimes U_{\mathrm{V}}(1) \otimes$ $U_{\mathrm{A}}(1)$ transformations is composed of eight channels. ${ }^{5}$

\section{FRAMEWORK}

\section{A. Four-fermion interactions and phase transitions: Brief introduction}

Before we actually analyze the fixed-point and phase structure of our model at finite temperature and quark chemical potential, we briefly discuss our RG framework. A detailed discussion of the latter can be found in Ref. [10], of which this work is a sequel. Moreover, we also give only a brief discussion of how a study of the quantum effective action (6) at leading order in the derivative expansion can give us access to the phase structure. A detailed discussion of this issue is also given in Refs. [8,10].

We begin our discussion by noting that the four-quark vertex is treated in the so-called pointlike limit at leading order of the derivative expansion, i.e., in the limit of vanishing external momenta. It is then clear that the mass spectrum encoded in the momentum structure of, e.g., the general four-quark vertex cannot be accessed at this order. In particular, the dynamics of regimes governed by the spontaneous formation of condensates is not accessible at this order. In fact, the formation of such condensates is indicated by singularities in the four-quark correlation functions. Nevertheless, the effective action (6) at leading

\footnotetext{
${ }^{5}$ Albeit possible, we do not use a basis of four-quark channels composed of an eight-dimensional subspace invariant under $S U\left(N_{\mathrm{c}}\right) \otimes S U_{\mathrm{L}}(2) \otimes S U_{\mathrm{R}}(2) \otimes U_{\mathrm{V}}(1) \otimes U_{\mathrm{A}}(1) \quad$ transformations and a remaining two-dimensional subspace only invariant under $S U\left(N_{\mathrm{c}}\right) \otimes S U_{\mathrm{L}}(2) \otimes S U_{\mathrm{R}}(2) \otimes U_{\mathrm{V}}(1)$ transformations in order to make better contact with conventional QCD model studies.
} 
order in the derivative expansion still allows us to study regimes which are not governed by condensate formation, e.g., the high-temperature regime. For a given value of the quark chemical potential, we can therefore use the leading order of the derivative expansion to determine a critical temperature $T_{\mathrm{cr}}$ below which the pointlike approximation breaks down and a (fermion) condensate associated with the spontaneous breakdown of one of the symmetries of our model is expected to be generated dynamically. This can also be understood from the standpoint of a HubbardStratonovich transformation [32]. Using such a transformation, we can reformulate our purely fermionic action in terms of quark fields and auxiliary bosonic fields (composites of two fermion fields, e.g., pionlike field or diquarklike field) coupled via Yukawa-type interactions. Such a reformulation eventually allows us to compute conveniently the GinzburgLandau-type effective potential for the bosonic fields. The transformation properties of the latter depend on the tensor structure of the corresponding four-quark channel. Now, in case of a second-order phase transition, the sign of at least one of the terms bilinear in the bosonic fields appearing in the Ginzburg-Landau-type effective potential changes. At a firstorder phase transition, the situation is different. Still, taking into account all quantum fluctuations, the Ginzburg-Landautype effective potential becomes also convex in this case, implying that at least one of the masslike parameters associated with the terms bilinear in the bosonic fields tends to zero in the long-range limit. Since the pointlike four-quark couplings are found to be inverse proportional to the masslike parameters associated with the bosonic fields, a diverging four-quark coupling in the purely fermionic formulation indicates the onset of spontaneous symmetry breaking.

With respect to the RG analysis underlying the present work, these considerations imply that the observation of a divergence of a four-quark coupling at an RG scale $k_{\text {cr }}$ can be used as an indicator for the onset of spontaneous symmetry breaking. Such an analysis has indeed been successfully applied to compute the phase structure of various systems including gauge theories with many flavors (see, e.g., Ref. [33]); see Ref. [8] for a review. However, it should also be noted that this type of analysis is limited. ${ }^{6}$ For example, it does not allow us to resolve the order of a phase transition. Moreover, the phenomenological meaning of a critical temperature obtained from such an analysis is potentially ambiguous. Different symmetry breaking patterns associated with the various four-quark channels exist in our model. Therefore, it is at least difficult to relate the breakdown of the pointlike approximation to the spontaneous breakdown of a specific symmetry, even more so since a divergence in a specific four-quark channel entails corresponding divergences in all other channels. However, a "dominantly diverging" four-quark channel can in general

\footnotetext{
${ }^{6}$ For a detailed discussion of such an analysis and its limitations, we refer the reader to Refs. $[8,10]$.
}
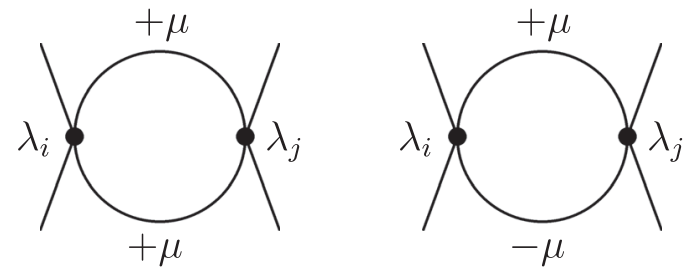

FIG. 1. The two classes of 1PI diagrams contributing to the RG flow of the four-quark couplings.

be identified; i.e., the modulus of the coupling of this channel is greater than the ones of the other four-quark couplings. Of course, this does not necessarily imply that a condensate associated with this channel is generated. It should only be viewed as an indicator for the symmetry breaking scenario at work. In Sec. IV, we present an analysis of the "hierarchy" of the various four-quark interactions in terms of their strength and show that our "criterion of dominance" is at least in accordance with the simplest phenomenological expectation of the symmetry breaking patterns at work at small and large chemical potentials [5]. We have checked that our results from such an analysis are not altered when we rescale the channels (7)-(16) with factors of $\mathcal{O}(1)$.

For the computation of the RG flows of the four-quark couplings, we employ the Wetterich equation [34], which is an RG equation for the quantum effective action $\Gamma$. Within this framework, the effective action $\Gamma$ depends on the RG scale $k$, which is related to the RG "time" $t=\ln (k / \Lambda)$. Here, the scale $k$ defines an IR cutoff scale, and $\Lambda$ may be chosen to be the scale at which we fix the initial conditions of the RG flow of the effective action. In this RG approach, the Wilsonian momentum-shell integrations are specified by the regularization scheme, which is encoded in the form of a so-called regulator function. Following Ref. [10], we employ here a so-called four-dimensional Fermi-surface adapted regularization scheme, which becomes manifestly covariant in the limit $T \rightarrow 0$ and $\mu \rightarrow 0$; see also Appendix A.

In general, we then find that the RG flow of the fourquark couplings is governed by two classes ${ }^{7}$ of one-particle irreducible (1PI) diagrams; see Fig. 1. These diagrams can be recast into so-called threshold functions which describe the decoupling of massive modes and modes in a thermal and/or dense medium. The definitions of these functions can be found in Ref. [10].

\section{B. Scale-fixing procedure}

Let us now give a detailed discussion of the scale-fixing procedure underlying our calculations in this work. The values of the ten four-quark couplings at the initial RG scale $k=\Lambda$ can be considered as free parameters of our model. To

\footnotetext{
${ }^{7}$ The two classes contain diagrams which are associated with contributions longitudinal and transversal to the heat bath.
} 
pin them down, let us consider RG studies of QCD in which the strengths of pointlike gluon-induced four-quark interactions have been analyzed in detail in the vacuum limit within a Fierz-complete setting [35-37]. There, it was found that the scalar-pseudoscalar channel $\mathcal{L}_{(\sigma-\pi)}$ is generated predominantly at high-momentum scales $p \sim k$. Moreover, it was found that this channel remains to be the most dominant one over a wide range of scales down to $k \gtrsim 1 \mathrm{GeV}$; i.e., the modulus of any other four-quark coupling remains smaller than the one of the scalar-pseudoscalar coupling. With respect to our present study, it is also reasonable to expect that effects associated with an explicit breaking of Poincare and $\mathcal{C}$ invariance are subleading as long as $T / k \ll 1$ and $\mu / k \ll 1$. In the light of these facts, we only choose the scalar-pseudoscalar coupling $\bar{\lambda}_{(\sigma-\pi)}$ to be finite at the initial RG scale $\Lambda$ and set all other four-quark couplings to zero. Thus, at the initial scale, we are left with the action $S$ given in Eq. (1), $\Gamma_{k=\Lambda}=S$. This implies that we assume the $U_{\mathrm{A}}(1)$ symmetry to be broken explicitly at the initial RG scale. ${ }^{8}$ Clearly, these considerations do not represent a rigorous determination of the initial conditions of our NJL-type model from QCD, which would require the dynamical inclusion of gauge d.o.f. in an RG study [33,35,36,38], but rather serve as a motivation for our scale-fixing procedure below.

The initial condition of the remaining coupling, the scalarpseudoscalar coupling $\bar{\lambda}_{(\sigma-\pi)}$, can be fixed in different ways. For example, we may tune it in the vacuum limit such that the resulting symmetry breaking scale $k_{\text {cr }}$ leads to a given value for the critical temperature at vanishing chemical potential [10]. In the following, however, we employ a different procedure which exploits the mean-field gap equation for the chiral order-parameter field in a scalar-pseudoscalar onechannel approximation. To be more specific, performing a Hubbard-Stratonovich transformation of the path integral defined by the action (1) and integrating out the quark d.o.f. subsequently, we arrive at a path integral over the auxiliary fields $\sigma \sim(\bar{\psi} \gamma)$ and $\vec{\pi} \sim\left(\bar{\psi} \gamma_{5} \vec{\tau} \psi\right)$, respectively. From an evaluation of the latter in the mean-field approximation, we then obtain the implicit equation for the constituent quark mass $\bar{m}_{\mathrm{q}}^{2}=\langle\sigma\rangle^{2}$ at $T=\mu=0,{ }^{9}$

$$
\lambda_{(\sigma-\pi)}^{*} \mathcal{J}(0)=\lambda_{(\sigma-\pi)}^{(\mathrm{UV})} \mathcal{J}\left(\bar{m}_{\mathrm{q}}^{2}\right),
$$

where $\lambda_{(\sigma-\pi)}^{\mathrm{UV}}=\Lambda^{2} \bar{\lambda}_{(\sigma-\pi)}^{(\mathrm{UV})}$ and $\lambda_{(\sigma-\pi)}^{*} \equiv \lambda_{(\sigma-\pi)}^{*}\left[r_{\psi}\right]$ is a dimensionless functional of the regularization scheme since $\mathcal{J}$ is not only a function of $\bar{m}_{\mathrm{q}}$ but also a functional of the so-called regulator shape function $r_{\psi}$ specifying the regularization scheme,

\footnotetext{
${ }^{8}$ In Sec. IV C, we discuss the effect of $U_{\mathrm{A}}(1)$ symmetry breaking in more detail with the aid of the sum rules (17) and (18).

${ }^{9}$ From now on, we identify $\bar{\lambda}_{(\sigma-\pi)}^{(\mathrm{UV})}$ with the value of the coupling $\bar{\lambda}_{(\sigma-\pi)}$ appearing in the classical action since the latter determines the value of this coupling at the UV scale $\Lambda$ in our RG study below.
}

$\mathcal{J}\left(\bar{m}_{\mathrm{q}}^{2}\right)=8 N_{\mathrm{c}} \int \frac{\mathrm{d}^{4} p}{(2 \pi)^{4}}\left(\frac{1}{p^{2}+\bar{m}_{\mathrm{q}}^{2}}-\frac{1}{p^{2}\left(1+r_{\psi}\left(\frac{p^{2}}{\Lambda^{2}}\right)\right)^{2}+\bar{m}_{\mathrm{q}}^{2}}\right) ;$

see also Ref. [8] for details. Diagrammatically, this integral is associated with a purely fermionic loop integral evaluated at vanishing external momenta. ${ }^{10}$ The parameter $\Lambda$ may be considered a UV cutoff scale for the loop-momentum integral. However, from our RG standpoint, it should be rather associated with the initial RG scale at which we fix the initial conditions of the four-quark couplings in our RG study below.

For a given regularization scheme, the functional $\lambda_{(\sigma-\pi)}^{*}$ determines the critical value of the four-quark coupling above which the ground state is governed by a finite vacuum expectation value $\langle\sigma\rangle \neq 0$. We find

$$
\lambda_{(\sigma-\pi)}^{*}=\frac{\Lambda^{2}}{\mathcal{J}(0)} .
$$

Thus, we have $\bar{m}_{\mathrm{q}}>0$ for $\lambda_{(\sigma-\pi)}^{\mathrm{UV}}>\lambda_{(\sigma-\pi)}^{*}$ and $\bar{m}_{\mathrm{q}}=0$ otherwise. For example, we obtain $\lambda_{(\sigma-\pi)}^{*}=2 \pi^{2} / N_{\mathrm{c}}$ for the four-dimensional sharp cutoff often employed in mean-field calculations and $\lambda_{(\sigma-\pi)}^{*}=4 \pi^{2} / N_{\mathrm{c}}$ for the Litim regulator [39]. From here on, however, we shall employ the same scheme as in our studies of the RG flow of four-quark couplings to ensure comparability; see Appendix A for details. For this scheme, we find $\lambda_{(\sigma-\pi)}^{*}=2 \pi^{2} / N_{\mathrm{c}}$. In any case, we deduce from Eq. (19) that the actual value of $\lambda_{(\sigma-\pi)}^{*}$ is of no importance. For a given regularization scheme together with a specific choice for the UV scale $\Lambda$, the quark mass $\bar{m}_{\mathrm{q}}$ only depends on the "strength" $\Delta \lambda_{(\sigma-\pi)}$ of the scalarpseudoscalar coupling relative to its critical value for chiral symmetry breaking:

$$
\Delta \lambda_{(\sigma-\pi)}=\frac{\lambda_{(\sigma-\pi)}^{(\mathrm{UV})}-\lambda_{(\sigma-\pi)}^{*}}{\lambda_{(\sigma-\pi)}^{(\mathrm{UV})}} .
$$

In the following, we shall fix the scale in our studies by setting $\bar{m}_{\mathrm{q}} \approx 0.300 \mathrm{GeV}$ for the constituent quark mass in order to relate our model study to QCD. In terms of the scalar-pseudoscalar coupling, this choice corresponds to $\Delta \lambda_{(\sigma-\pi)} \approx 0.234$ for $\Lambda / \bar{m}_{\mathrm{q}} \approx 10 / 3$.

From this discussion, it follows immediately that a specific choice for $\Delta \lambda_{(\sigma-\pi)}$ also determines the sign of the curvature $\bar{m}^{2}$ of the order-parameter potential $U$ at the origin. Indeed, we have

\footnotetext{
${ }^{10}$ Loosely speaking, the loop integral corresponds to the diagram shown in the left panel of Fig. 1 with amputated external fermionic legs.
} 


$$
\bar{m}^{2}:=\left.2 \frac{\partial U}{\partial \sigma^{2}}\right|_{\sigma=0}=-\Lambda^{2} \frac{\Delta \lambda_{(\sigma-\pi)}}{\lambda_{(\sigma-\pi)}^{*}},
$$

implying that, at the "critical point" $\Delta \lambda_{(\sigma-\pi)}=0$, the curvature $\bar{m}^{2}$ of the order-parameter potential changes its sign. From the underlying Hubbard-Stratonovich transformation, we deduce that the renormalized scalar-pseudoscalar coupling $\bar{\lambda}_{(\sigma-\pi)}$ is inverse proportional to the curvature $\bar{m}^{2}$ :

$$
\Lambda^{2} \bar{\lambda}_{(\sigma-\pi)}=\frac{\Lambda^{2}}{\bar{m}^{2}}=-\frac{\lambda_{(\sigma-\pi)}^{*}}{\Delta \lambda_{(\sigma-\pi)}} .
$$

Thus, the scalar-pseudoscalar four-quark coupling diverges at the critical point $\Delta \lambda_{(\sigma-\pi)}=0$.

As discussed on more general grounds in Sec. III A, these observations regarding the critical behavior and the formation of a nontrivial ground state can be carried over to studies of the RG flow of four-quark interactions, even beyond the mean-field limit. We refer the reader to Ref. [8] for a corresponding detailed discussion in which it has also been shown that the value of $\lambda_{(\sigma-\pi)}^{*}$ in the mean-field approximation is indeed nothing but the value of the non-Gaussian fixed point of the scalar-pseudoscalar coupling in the large- $N_{\mathrm{c}}$ limit. Thus, this non-Gaussian fixed point separates a regime governed by a trivial ground state from one governed by spontaneous symmetry breaking.

Let us now exploit the relation between the orderparameter potential and the RG flow of four-quark couplings to fix the scale in our study of the phase diagram below. To be specific, we first consider the flow equation for the dimensionless scale-dependent renormalized scalarpseudoscalar coupling $\lambda_{(\sigma-\pi)}=Z_{(\sigma-\pi)} k^{2} \bar{\lambda}_{(\sigma-\pi)} /\left(Z^{\perp}\right)^{2}$ in the one-channel approximation at $T=\mu=0$ :

$$
\partial_{t} \lambda_{(\sigma-\pi)}=2 \lambda_{(\sigma-\pi)}-\frac{N_{\mathrm{c}}}{\pi^{2}}\left(1+\frac{1}{2 N_{\mathrm{c}}}\right) \lambda_{(\sigma-\pi)}^{2} .
$$

Here, we have employed the regularization scheme defined in Appendix A, which is identical to the well-known fourdimensional exponential scheme in the vacuum limit [40,41]; see Ref. [42] for a detailed discussion of regularization schemes in RG studies. The flow equation (25) has been extracted from the set of equations for the Fierz-complete basis of four-quark interactions given in Appendix B by setting all couplings but the scalar-pseudoscalar coupling to zero and also dropping their flow equations. Note that, because of the Fierz ambiguity, the flow equation (25) is ambiguous in the sense that the prefactor of the term quadratic in the four-quark coupling is not unique.

The flow equation (25) has two fixed points: a Gaussian fixed point and the aforementioned non-Gaussian fixed point $\lambda_{(\sigma-\pi)}^{*}$,

$$
\lambda_{(\sigma-\pi)}^{*}=\frac{2 \pi^{2}}{N_{\mathrm{c}}+\frac{1}{2}} .
$$

Thus, the value of the non-Gaussian fixed point indeed agrees with the critical value of the scalar-pseudoscalar coupling in the mean-field approximation for $N_{\mathrm{c}} \gg 1$. Again, with respect to the question of the formation of a nontrivial ground state, the actual value of the non-Gaussian fixed point is of no importance; only the value of the scalarpseudoscalar coupling at the initial $R G$ scale $\Lambda$ relative to the value of the non-Gaussian fixed point matters. Choosing $\lambda_{(\sigma-\pi)}^{(\mathrm{UV})}>\lambda_{(\sigma-\pi)}^{*}$, we find that the scalar-pseudoscalar coupling diverges at a finite scale $k_{\mathrm{cr}}$,

$$
k_{\mathrm{cr}}=\Lambda\left(\Delta \lambda_{(\sigma-\pi)}\right)^{\frac{1}{2}} \theta\left(\Delta \lambda_{(\sigma-\pi)}\right),
$$

indicating the onset of chiral symmetry breaking; i.e., the curvature of the order parameter at the origin changes its sign at this so-called chiral symmetry breaking scale $k_{\mathrm{cr}}$. This scale sets the scale for the (chiral) low-energy observables, such as the constituent quark mass $\bar{m}_{\mathrm{q}} \sim k_{\mathrm{cr}}$. Using the relation (27), we can compute the value of the chiral symmetry breaking scale in the mean-field approximation. Using $\Delta \lambda \approx 0.234$ extracted from the mean-field calculation above for $\bar{m}_{\mathrm{q}} \approx 0.300 \mathrm{GeV}$ and $\Lambda / \bar{m}_{\mathrm{q}} \approx 10 / 3$, we obtain $k_{0} / \bar{m}_{\mathrm{q}} \equiv k_{\mathrm{cr}} / \bar{m}_{\mathrm{q}} \approx 1.613$, where $k_{0}$ serves as a reference scale in the remainder of this work.

At first glance, it seems that Eq. (27) defining $k_{\mathrm{cr}}$ implies that the low-energy dynamics is independent of the combinatoric prefactor of the term quadratic in the fourquark coupling in Eq. (25). However, this turns out to be too naive. A closer look reveals that the contribution $\sim 1 / N_{\mathrm{c}}$ is related to quantum corrections to the Yukawa coupling in a partially bosonized formulation of our model $[8,43]$. In a study of the partially bosonized formulation, these corrections therefore yield $1 / N_{\mathrm{c}}$ corrections to the critical scale $k_{\mathrm{cr}}$. Moreover, it should be noted that order-parameter fluctuations, which are nothing but $1 / N_{\mathrm{c}}$ corrections, tend to restore the chiral symmetry in the infrared limit, thereby lowering the value of the critical temperature compared to its value in the large- $N_{\mathrm{c}}$ approximation (see, e.g., Ref. [44]).

At the order of the derivative expansion considered in this work, we do not have access to low-energy observables such as the constituent quark mass; see our discussion in Sec. III A. Therefore, we exploit the relation between the chiral order-parameter potential in the mean-field approximation and the RG flow of the corresponding scalar-pseudoscalar coupling to fix the scale in our calculations. In all our studies of the phase diagram presented below, we shall set all fourquark couplings to zero at the initial RG scale $\Lambda$ except for the scalar-pseudoscalar coupling $\lambda_{(\sigma-\pi)}$. The latter is tuned at this scale such that, at $T=\mu=0$, we obtain $k_{\mathrm{cr}}=k_{0}$, i.e., the value of the critical scale is always tuned to agree identically with its value in the mean-field approximation. This ensures comparability between the results of our studies from different approximations. Moreover, since $k_{0}$ is directly related to the constituent quark mass in the meanfield approximation, $k_{0} / \bar{m}_{\mathrm{q}} \approx 1.613$, this allows at least a 
rough translation of our results for the phase transition temperatures as obtained from, e.g., our Fierz-complete set of flow equations into physical units. Of course, such a translation is only approximative. We always have to keep in mind that the use of the same value for $k_{0}$ in different approximations may not necessarily translate into the same value for the low-energy observables, such as the constituent quark mass. In any case, considering the critical temperature at $\mu=0$ as an example for a low-energy observable being sensitive to the vacuum constituent quark mass and also accessible within our framework, we find that this quantity does not depend strongly on our approximations associated with different numbers of four-quark channels. This observation may be traced back to the fact that we find the scalar-pseudoscalar channel to be most dominant at $\mu=0$, therefore governing the low-energy dynamics in this regime; see our discussion below.

\section{FIXED-POINT AND PHASE STRUCTURE}

\section{A. Mean-field and one-channel approximation}

Let us now study the phase diagram in the plane spanned by the temperature and quark chemical potential for an approximation in which we only take into account the scalar-pseudoscalar four-quark coupling. The flow equation of the latter reads

$$
\begin{aligned}
\partial_{t} \lambda_{(\sigma-\pi)}= & 2 \lambda_{(\sigma-\pi)}-64 v_{4}\left(2 N_{\mathrm{c}}+1\right) \lambda_{(\sigma-\pi)}^{2}\left(l_{\|+}^{\mathrm{F})}\left(\tau, 0,-i \tilde{\mu}_{\tau}\right)\right. \\
& \left.+l_{\perp+}^{\mathrm{F})}\left(\tau, 0,-i \tilde{\mu}_{\tau}\right)\right),
\end{aligned}
$$

where $v_{4}=1 /\left(32 \pi^{2}\right), \quad \tau=T / k$, and $\tilde{\mu}_{\tau}=\mu /(2 \pi T)=$ $\mu /(2 \pi k \tau)$. Here and in the following, we do not take into account the renormalization of the chemical potential and set $Z_{\mu}=1$. The definitions of the so-called threshold functions also mentioned in Sec. III A can be found in Ref. [10]. The ones appearing in Eq. (28) are associated with the loop integral depicted in the left panel of Fig. 1. As was done for Eq. (25), we have extracted the flow equation (28) from the set of equations for the Fierz-complete basis of four-quark interactions given in Appendix B by setting all but the scalarpseudoscalar coupling to zero and also dropping their RG flow equations. In the limits $T \rightarrow 0$ and $\mu \rightarrow 0$, we therefore recover Eq. (25) from Eq. (28) since

$$
\left(l_{\|+}^{\mathrm{F})}\left(\tau, 0,-i \tilde{\mu}_{\tau}\right)+l_{\perp+}^{(\mathrm{F})}\left(\tau, 0,-i \tilde{\mu}_{\tau}\right)\right) \rightarrow \frac{1}{4}
$$

for $T \rightarrow 0$ and $\mu \rightarrow 0$; see Ref. [10].

The flow equation (28) can be solved analytically, even at finite temperature and quark chemical potential [10]. The solution can then be employed to compute the critical temperature $T_{\mathrm{cr}}=T_{\mathrm{cr}}(\mu)$ as a function of the quark chemical potential $\mu$. The latter is defined as the temperature at which the scalar-pseudoscalar four-quark coupling still diverges at $k \rightarrow 0$,

$$
\lim _{k \rightarrow 0} \frac{1}{\lambda_{(\sigma-\pi)}\left(T_{\mathrm{cr}}, \mu, k\right)}=0
$$

i.e., it is defined as the highest temperature for which the four-quark coupling still diverges. For our studies with more than one channel, this definition can be generalized straightforwardly. The critical temperature is then defined to be the temperature at which the four-quark couplings diverge. Note that a divergence in one channel at a scale $k_{\mathrm{cr}}(T, \mu)$ entails corresponding divergences in all the other channels at the same scale. However, the associated fourquark couplings in general have a different strength relative to each other; see, e.g., Sec. IV B below and also Ref. [10] for a detailed discussion.

With the definition (30), we obtain the following implicit equation for the critical temperature $T_{\mathrm{cr}}$ :

$$
k_{0}=\Lambda\left(1+8 \mathcal{I}\left(T_{\mathrm{cr}}, \mu, 0\right)\right)^{\frac{1}{2}} .
$$

For convenience, we have introduced the dimensionless auxiliary function

$$
\begin{aligned}
\mathcal{I}(T, \mu, k)= & \frac{1}{\Lambda^{2}} \int_{\Lambda}^{k} \mathrm{~d} k^{\prime} k^{\prime}\left(l_{\|+}^{(\mathrm{F})}\left(\tau^{\prime}, 0,-i \tilde{\mu}_{\tau^{\prime}}\right)\right. \\
& \left.+l_{\perp+}^{(\mathrm{F})}\left(\tau^{\prime}, 0,-i \tilde{\mu}_{\tau^{\prime}}\right)\right),
\end{aligned}
$$

where $\tau^{\prime}=T / k^{\prime}$.

Apparently, the critical temperature $T_{\text {cr }}$ depends on our choice for the UV scale $\Lambda$ as well as the scale $k_{0}$, i.e., eventually on the constituent quark mass in the vacuum limit. Recall that the scale $k_{0}$ is directly related to the initial condition for the scalar-pseudoscalar four-quark coupling, which we keep fixed to the same value for all temperatures and chemical potentials. In the following, we shall therefore measure all physical observables in units of $k_{0}$.

In Fig. 2, we show the critical temperature as a function of the quark chemical potential for the one-channel approximation (gray line) as obtained from a solution of Eq. (31). For $\mu=0$, we obtain $T_{\text {cr }} / k_{0} \approx 0.390\left(T_{\text {cr }} \approx 0.190 \mathrm{GeV}\right)$. For increasing $\mu$, the critical temperature then decreases monotonously and eventually vanishes at $\mu / k_{0}=\mu_{\mathrm{cr}} / k_{0} \approx$ $1.14\left(\mu_{\mathrm{cr}} \approx 0.552 \mathrm{GeV}\right)$.

We emphasize again that our definition of the critical temperature is associated with a sign change of the chiral order-parameter potential at the origin. In our present approximation, our result for $T_{\mathrm{cr}}(\mu)$ therefore only describes the phase boundary in case of a second-order transition. Our criterion is not sensitive to a first-order transition. However, it allows us to detect the line of metastability separating a regime associated with a negative curvature of the order-parameter potential at the origin (e.g., at low temperature and small quark chemical potential) from a regime in which the curvature changes its sign but the true ground state is still assumed for a finite expectation value of the order-parameter field. Such lines 


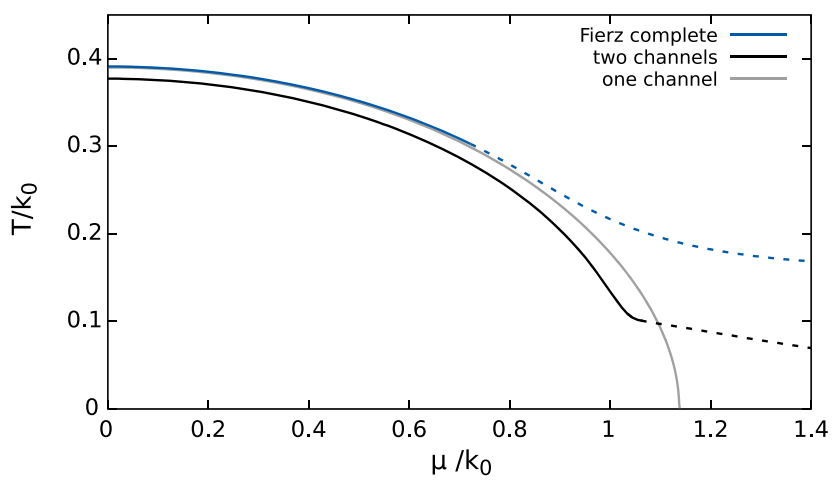

FIG. 2. Phase boundary associated with the spontaneous breakdown of at least one of the fundamental symmetries of our NJLtype model as obtained from a one-channel, two-channel, and Fierz-complete study of the Ansatz (6); see the main text for details.

of metastability usually emerge in the vicinity of a firstorder transition. In particular, for a given temperature, the chemical potential associated with the emergence of a metastable state at the origin of the potential is less than or equal to the chemical potential of the associated first-order transition; see Ref. [45] for the first NJL model analysis of this aspect.

It is instructive to compare the results for the phase boundary from our RG study with those obtained from a solution of the mean-field gap equation (19). ${ }^{11}$ To ensure comparability, we employ of course the same regularization scheme as in our RG study. We then find that the phase transition line from our RG study agrees identically with the second-order phase transition line of the mean-field study up to a first-order end point at $\left(\mu / k_{0}, T / k_{0}\right) \approx(0.951,0.207)$. As expected, beyond this point, the phase transition line obtained from our RG study agrees identically with the line of metastability in the mean-field phase diagram. The comparatively large extent of the phase boundary in the $\mu$ direction can be traced back to the comparatively large $\sigma$-meson mass $\bar{m}_{\sigma} / \bar{m}_{\mathrm{q}} \approx 2.67$ $\left(\bar{m}_{\sigma} \approx 0.800 \mathrm{GeV}\right.$ ) found in the vacuum limit of our meanfield calculation for the employed set of parameters, i.e., $\Delta \lambda_{(\sigma-\pi)}$ and $\Lambda .{ }^{12}$ In fact, it has already been found in previous mean-field calculations that the critical point separating a first-order phase transition line from a second-order phase transition line can be shifted continuously to larger values of the quark chemical potential by increasing the mass of the $\sigma$ meson [46]. Even more, it

\footnotetext{
${ }^{11}$ The gap equation for finite $T$ and $\mu$ is obtained from Eq. (19) by replacing $\int \mathrm{d}^{4} p /(2 \pi)^{4}$ with $T \sum_{n} \int \mathrm{d}^{3} p /(2 \pi)^{3}$. Moreover, we have to replace $p^{2}$ with $\left(\nu_{n}^{2}-\mathrm{i} \mu\right)^{2}+\vec{p}^{2}$, where $\nu_{n}=(2 n+1) \pi T$, except in the argument of the regulator shape function due to our conventions.

${ }^{12}$ The computation of the $\sigma$ mass requires fixing the Yukawa coupling $\bar{h}$ since $m_{q}=\langle\sigma\rangle=\bar{h} f_{\pi}$. Here, we use $\bar{h} \approx 3.45$ corresponding to $f_{\pi} \approx 87.0 \mathrm{MeV}$ for the pion decay constant.
}

can even be made to disappear, leaving us with only a second-order transition line, depending on the actual choice of the model parameters [47]. Note that this highlights the strong scheme dependence as the $\sigma$ mass can be tuned by suitable variations of the constituent quark mass and the UV cutoff $\Lambda$. Since the actual value of the latter should always be viewed against the background of the employed regularization scheme, the scheme unavoidably belongs to the definition of the model, at least in four Euclidean spacetime dimensions. Of course, we could also use smaller values for $\Lambda$, which would lead to a smaller mass of the $\sigma$ meson. However, this then leads to strong "cutoff effects" as both the temperatures as well as the quark chemical potentials considered in this work would then be of the order of the UV scale $\Lambda$. To at least suppress such unwanted effects, we have chosen $\Lambda / k_{0} \approx 2.07$.

\section{B. Symmetry breaking patterns and Fierz completeness}

Let us now analyze the phase diagram as obtained from an RG flow study of the Fierz-complete set of four-quark interactions; see Appendix B for the RG flow equations. Such an analysis goes well beyond studies in the mean-field limit. Indeed, mean-field studies of NJL-type low-energy models have been found to exhibit a residual ambiguity related to the possibility to perform Fierz transformations, even if a Fierz-complete set of four-quark interactions is taken into account [48]; see also Ref. [49] for an introduction. Results from mean-field calculations therefore potentially depend on an unphysical parameter, which reflects the actual choice of the mean field in the various channels.

As discussed above, we fix the scale in our Fierz-complete studies by setting all but the scalar-pseudoscalar coupling to zero at the initial RG scale $\Lambda$. The latter is tuned at $T=$ $\mu=0$ such that the critical scale $k_{0}$ associated with diverging

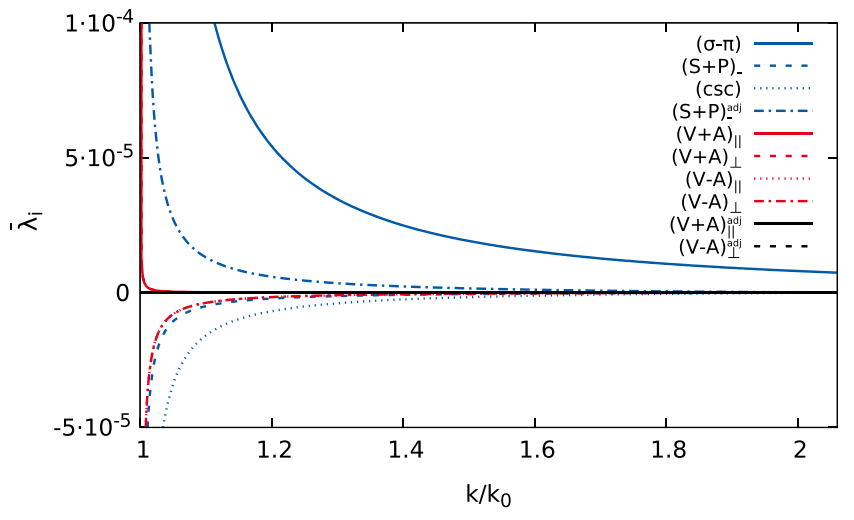

FIG. 3. Renormalized (dimensionful) four-quark couplings as a function of the RG scale $k$ at $T=0$ and $\mu=0$ as obtained from our Fierz-complete study. Note that the Fierz-complete basis is effectively composed of only six channels in the vacuum limit since $\mathcal{C}$ invariance is intact and the Euclidean time direction is not distinguished. In particular, we have $\bar{\lambda}_{(V+A)_{\|}^{\mathrm{adj}}} \equiv 0 \equiv \bar{\lambda}_{(V-A)_{\perp}^{\mathrm{adj}}}$ in this limit. 

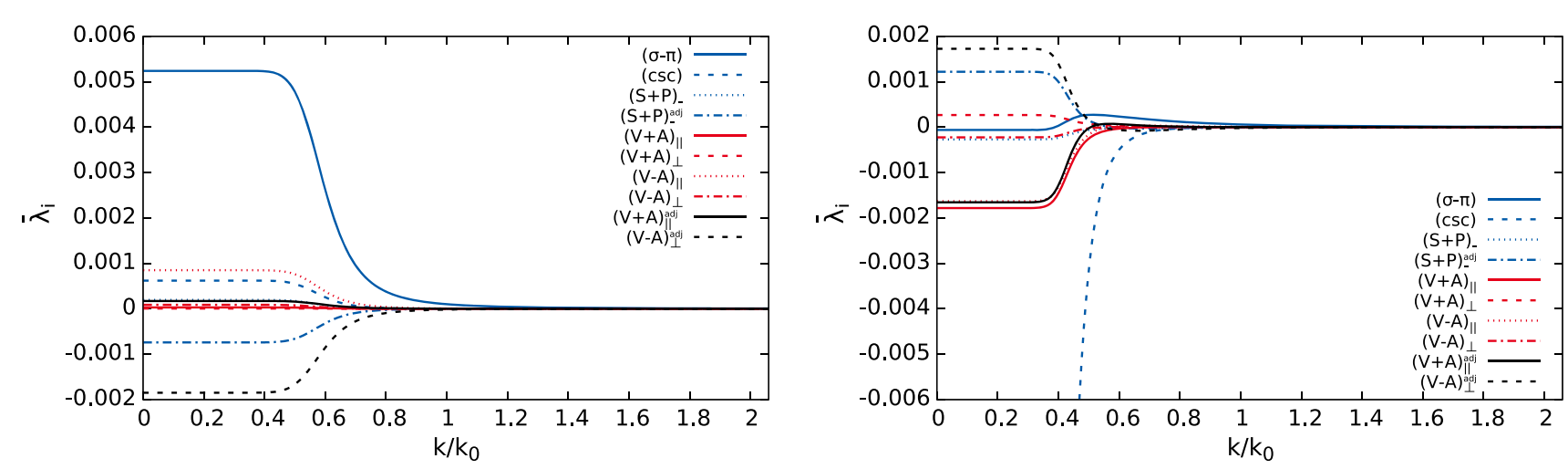

FIG. 4. Scale dependence of the various renormalized (dimensionful) four-quark couplings at $\mu=0$ and $T / k_{0} \simeq T_{\text {cr }}(\mu=0) / k_{0} \approx$ 0.391 (left panel) as well as at $\mu / k_{0} \approx 1.1$ and $T / k_{0} \simeq T_{\text {cr }}(\mu) / k_{0} \approx 0.196$ (right panel).

four-quark couplings agrees identically with its counterpart in the mean-field calculation. For our calculations at finite temperature and/or quark chemical potential, we then use the same set of initial conditions as in the vacuum limit, i.e., at $T=\mu=0$. The scale dependence of the (dimensionful) renormalized four-quark couplings at $T=\mu=0$ is shown in Fig. 3. We observe that the dynamics of the theory in this case is clearly dominated by the scalar-pseudoscalar interaction channel; the modulus of all other couplings is at least one order of magnitude smaller than the modulus of the scalarpseudoscalar coupling. The dominance of this channel may indicate that the ground state in the vacuum limit is governed by chiral symmetry breaking. However, we emphasize again that such an analysis based on the strength of four-quark interactions has to be taken with some care; it neither rules out the possible formation of other condensates associated with subdominant channels nor proves the formation of a condensate associated with the most dominant channel. Such an analysis can only yield indications for the actual structure of the ground state; see Ref. [10] for a detailed discussion.

In the vacuum limit, the observation of the dominance of the scalar-pseudoscalar channel may be considered trivial as it may be exclusively triggered by our choice for the initial conditions. Increasing now the temperature at vanishing quark chemical potential, we still observe a dominance of the scalar-pseudoscalar channel, which persists even up to high temperatures beyond the critical temperature $T_{\text {cr }}(\mu=0) / k_{0} \approx 0.391$. This is illustrated in the left panel of Fig. 4, in which the scale dependence of the various four-quark couplings is shown for $T \simeq T_{\mathrm{cr}}(\mu=0)$ at $\mu=0$. At least in units of $k_{0}$, it also appears that the critical temperature at $\mu=0$ in our Fierz-complete study agrees very well with the one from the one-channel approximation. However, we note that this could be misleading as choosing the same value for $k_{0}$ in our Fierz-complete study and in our one-channel approximation may not necessarily lead to the same values of the low-energy observables (e.g., the constituent quark mass), although the flow in the vacuum limit is also strongly dominated by the scalarpseudoscalar channel in the Fierz-complete analysis. Thus, direct quantitative comparisons of the results from our various different approximations should be taken with care. Still, we expect that qualitative comparisons are meaningful.

Following now the critical temperature $T_{\mathrm{cr}}$ as a function of $\mu$ starting from $\mu=0$, we find that the scalar-pseudoscalar channel continues to dominate the dynamics up to $\mu / k_{0}=\mu_{\chi} / k_{0} \approx 0.734$, as depicted by the blue solid line in Fig. 2. In this regime, we also observe that the phase transition temperatures from our one-channel approximation agree almost identically with those from the Fierzcomplete study, at least in units of the vacuum symmetry breaking scale $k_{0} \cdot{ }^{13}$ At first glance, this may come as a surprise. We shall therefore analyze this observation in detail in Sec. IV D below. Following the phase transition line beyond the point associated with the quark chemical potential $\mu_{\chi}$, we find that the dynamics is now clearly and exclusively dominated by the color-superconducting (CSC) channel associated with the emergence of a diquark condensate $\delta^{a}$ and a corresponding gap in the quark propagator; see the blue dashed line in Fig. 2. Exemplary, this change in the hierarchy of the channels is illustrated in the right panel of Fig. 4, in which the scale dependence of the various four-quark couplings is shown for $\mu / k_{0} \approx 1.1$ and $T / k_{0} \gtrsim T_{\mathrm{cr}}(\mu) / k_{0} \approx 0.196$. We emphasize that this change in the hierarchy of the channels is nontrivial as it is fully triggered by the dynamics of the system when the quark chemical potential is increased. There is no fine-tuning of, e.g., the CSC coupling involved. Recall that we use identical initial conditions in the vacuum limit as well as at finite temperature and/or quark chemical potential. From a phenomenological standpoint, it is interesting to speculate

\footnotetext{
${ }^{13}$ Note that $\mu_{\chi} / k_{0} \approx 0.734$ roughly corresponds to $\mu_{\chi} / \bar{m}_{\mathrm{q}} \approx$ 1.18 in our mean-field approximation, where $\bar{m}_{\mathrm{q}} \approx 0.3 \mathrm{GeV}$.
} 

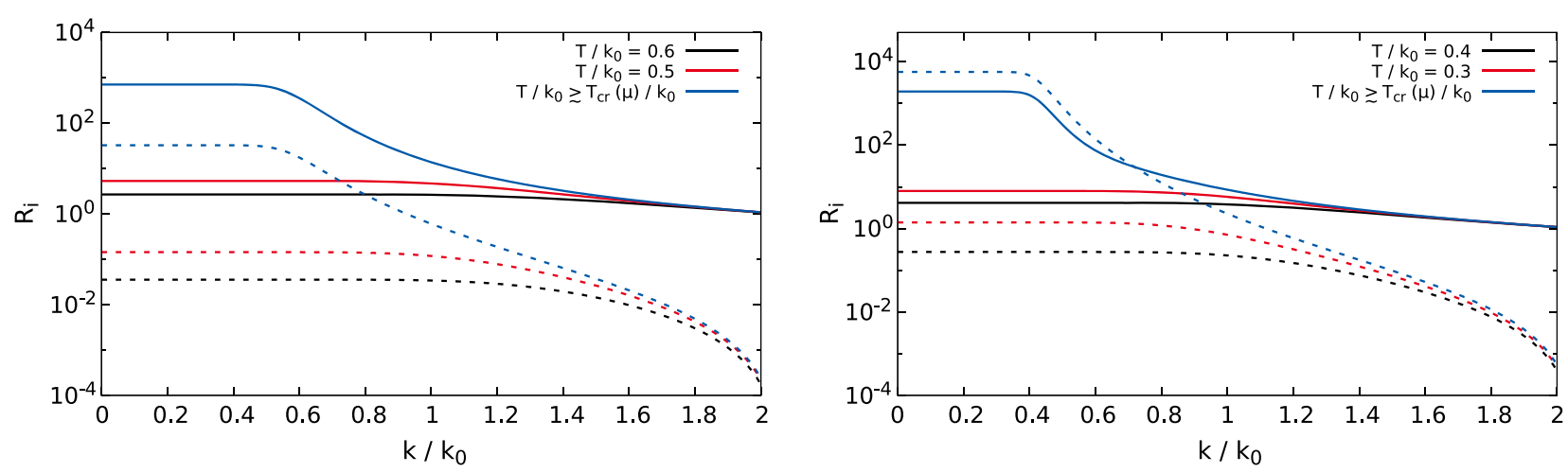

FIG. 5. Scale dependence of the explicit $U_{\mathrm{A}}(1)$ breaking as measured by the functions $R_{1}$ (dashed lines) and $R_{2}$ (solid lines) at $\mu=0$ (left panel) and at $\mu / k_{0} \approx 1.1$ (right panel) for three values of the temperature for each of the two cases.

whether such a change in the hierarchy of the channels points to the existence of a nearby tricritical point in the phase diagram. However, as discussed above, this question cannot be unambiguously resolved here because of the approximations underlying our present study.

\section{C. $U_{\mathrm{A}}(1)$ symmetry}

Our choice for the initial conditions of the RG flow equations explicitly breaks the $U_{\mathrm{A}}(1)$ symmetry since we only choose the coupling $\lambda_{(\sigma-\pi)}$ to be finite and set all the other four-quark couplings to zero at the initial RG scale $\Lambda$. By using the sum rules (17) and (18), we can now study the fate of the (broken) $U_{\mathrm{A}}(1)$ symmetry at finite temperature and quark chemical potential when quantum fluctuations are taken into account. To be specific, in case of $U_{\mathrm{A}}(1)$ violating initial conditions, we consider the following two dimensionless quantities to "measure" the strength of the explicit $U_{\mathrm{A}}(1)$ symmetry breaking:

$$
R_{i}=\mathcal{N}\left|\mathcal{S}_{U_{\mathrm{A}}(1)}^{(i)}\right| \cdot
$$

The normalization $\mathcal{N}$ is chosen to be independent of the index $i$ and is implicitly determined by

$$
1=\left.\left(R_{1}+R_{2}\right)\right|_{k=\Lambda} .
$$

Thus, the auxiliary quantities defined in Eq. (33) essentially measure the strength of $U_{\mathrm{A}}(1)$ symmetry breaking relative to its strength at the initial RG scale $\Lambda$. In case of a $U_{\mathrm{A}}(1)$ symmetric theory defined by a suitable choice for the initial conditions, we find that the couplings fulfill the sum rules (17) and (18) at all scales $k$ greater than the symmetry breaking scale, as it should be. Therefore, there is no need at all to consider the auxiliary quantities defined in Eq. (33) in such a scenario.

In Fig. 5, we show the scale dependence of $R_{1}$ and $R_{2}$ for two values of the quark chemical potential, $\mu=0$ and $\mu / k_{0} \approx 1.1$, and three values of the temperature for each of the two cases as obtained for our $U_{\mathrm{A}}(1)$-violating initial conditions. Let us first note that, for all values of $\mu$ considered in this work, we observe that $U_{\mathrm{A}}(1)$ breaking as measured by our sum rules in the form of $R_{1}$ and $R_{2}$ is continuously softened when the temperature is increased. More specifically, at $\mu=0$, for example, we already find that the strength of $U_{\mathrm{A}}(1)$ breaking remains on the level of its strength at the initial scale $\Lambda$ for temperatures $T / T_{\mathrm{c}} \gtrsim 2$; i.e., its strength remains on the level as present in the classical action in this temperature regime. A qualitatively similar behavior can also be observed at finite chemical potential when the temperature is increased; see Fig. 5. Indeed, the strength of $U_{\mathrm{A}}(1)$ symmetry breaking is controlled by the strength of the four-quark couplings. Quantum corrections to the latter are thermally suppressed at high temperature due to the presence of a thermal mass of the fermions. This explains our observations at high temperature.

Conversely, approaching the phase transition from above for a given value of the chemical potential $\mu$, we find that the violation of the $U_{\mathrm{A}}(1)$ symmetry becomes continuously stronger, in the sense that the functions $R_{1}$ and $R_{2}$ start to increase, eventually deviating significantly from their values at the initial RG scale. Thus, quantum corrections to the fourquark couplings appear to amplify $U_{\mathrm{A}}(1)$ symmetry breaking when the phase governed by spontaneous symmetry breaking is approached from above, provided that $U_{\mathrm{A}}(1)$ symmetry breaking is explicitly broken at the initial RG scale.

In accordance with our observation that the scalarpseudoscalar channel is most dominant at small chemical potential (see, e.g., the left panel of Fig. 4), we also note that $R_{2} \gg R_{1}$ in this part of the phase diagram; see the left panel of Fig. 5. For $\mu \gtrsim \mu_{\chi}$, the CSC channel then dominates the dynamics, and thus $R_{1}$ and $R_{2}$ are of the same order of magnitude as both depend on the CSC coupling; see the right panels of Figs. 4 and 5. Thus, our results suggest that the dynamically generated violation of the $U_{\mathrm{A}}(1)$ symmetry is driven by the dynamics of the pions at small chemical potential, whereas it is driven by the dynamics of diquark 
d.o.f. associated with the CSC channel at large chemical potential.

Let us finally compare the phase diagram obtained from our Fierz-complete study employing $U_{\mathrm{A}}(1)$ symmetry-violating initial conditions with the one obtained from a manifestly $U_{\mathrm{A}}(1)$-symmetric Fierz-complete study. The latter has been calculated by tuning the couplings $\bar{\lambda}_{(\sigma-\pi)}$ and $\bar{\lambda}_{(S+P)_{-}}$at the initial RG scale such that the sum rule (18) is fulfilled and the same value for the symmetry scale $k_{0}$ as in the case with $U_{\mathrm{A}}(1)$ symmetry-violating initial conditions is obtained in the vacuum limit. If we choose the initial conditions in this way, i.e., such that they respect the $U_{\mathrm{A}}(1)$ symmetry, then this symmetry remains intact in the RG flow for all values of the RG scale, at least for those values of the temperature and the quark chemical potential for which the fourquark couplings remain finite on all scales $k \leq \Lambda$. For values of the temperature and the quark chemical potential at which the four-quark couplings diverge at a finite scale $k_{\mathrm{cr}}(T, \mu)$, the sum rules (17) and (18) are only fulfilled for $k>k_{\mathrm{cr}}(T, \mu)$. Below the symmetry breaking scale, the $U_{\mathrm{A}}(1)$ symmetry may potentially be broken spontaneously, e.g., alongside with the chiral symmetry. However, this cannot be resolved in our present study.

As already discussed above, a quantitative comparison of the results from our $U_{\mathrm{A}}(1)$-symmetric calculation with the ones from our explicitly $U_{\mathrm{A}}(1)$-violating calculation is generically difficult and has to be taken with some care. Leaving this concern aside for a moment, we observe that the phase transition lines from both studies agree almost identically on the scale of the plot. Only for larger values of the quark chemical potential, we find that the two phase transition lines start to deviate from each other. In particular, we observe that, along the phase transition line, a chemical potential $\mu_{\chi} / k_{0}$ associated with a change in the hierarchy of the four-quark couplings exists in both cases. Even the corresponding values of $\mu_{\chi} / k_{0}$ agree almost identically. Even more, for $\mu<\mu_{\chi}$, we find that the scalar-pseudoscalar channel dominates the dynamics of the theory in both cases; see the solid lines in Fig. 6. For $\mu>\mu_{\chi}$, the dynamics is then dominated by the CSC channel in case of $U_{\mathrm{A}}(1)$ symmetry-violating initial conditions; see the blue dashed line in Fig. 6. In the case of our $U_{\mathrm{A}}(1)$-symmetric study, however, we have a dominance of the $(V+A)_{\|}^{\text {adj }}$ channel in this regime as depicted by the red dashed-dotted line in Fig. 6; see Eq. (11) for the definition of this channel. The condensate associated with this channel also breaks the color symmetry of our theory. In mean-field studies (see, e.g., Ref. [5] for a review), the appearance of a corresponding condensate has also been discussed. However, its generation has been found to be induced by a simultaneous formation of a color-symmetry breaking diquark condensate. In accordance with this, we observe that the four most dominant channels along the



FIG. 6. Phase boundary associated with the spontaneous breakdown of at least one of the fundamental symmetries of our NJLtype model as obtained from a manifestly $U_{\mathrm{A}}(1)$-symmetric Fierz-complete study of the Ansatz (6) (red lines) and from a Fierz-complete study with broken $U_{\mathrm{A}}(1)$ symmetry (blue lines); see the main text for details.

phase transition line for $\mu>\mu_{\chi}$ are the $(V+A)_{\|}^{\text {adj }}$, $(S+P)_{-}^{\text {adj }}$, CSC, and $(V-A)_{\perp}^{\text {adj }}$ channels in our present study. These channels are all associated with the formation of a color-symmetry breaking condensate. Apart from the $(V \pm A)_{\|}$channels, color-singlet channels are found to be subdominant in this part of the phase diagram. The observed difference in the dominance pattern at large chemical potential in the $U_{\mathrm{A}}(1)$-symmetric and $U_{\mathrm{A}}(1)$ violating calculation may point to the importance of explicit $U_{\mathrm{A}}(1)$ breaking for the formation of the conventional CSC ground state at intermediate and large values of the chemical potential as discussed in early seminal works on color superconductivity; see, e.g., Refs. [25,26,50,51].

By simply looking at the shape of the phase boundary, one may be tempted to conclude that $U_{\mathrm{A}}(1)$ breaking does not strongly affect the position of the phase transition line. However, this may be too bold a statement at this point, as the same value of $k_{0}$ in the two studies potentially corresponds to different values of the low-energy observables and therefore renders a direct quantitative comparison difficult; see our discussion above. In any case, the apparent insensitivity of the phase transition line under a "transition" from $U_{\mathrm{A}}(1)$ symmetry-violating initial conditions to $U_{\mathrm{A}}(1)$-symmetric initial conditions (while keeping the vacuum symmetry breaking scale fixed) is still an interesting observation. At least at small values of the chemical potential, the latter can in principle be understood from an analysis of the large- $N_{\mathrm{c}}$ limit, which we shall consider next.

\section{Large- $N_{c}$ limit}

To better understand the phase structure at small chemical potential, we now analyze our RG flow equations in the large- $N_{\mathrm{c}}$ limit; i.e., we only take into account the leading order of the right-hand sides of our flow equations in an expansion in powers of $N_{\mathrm{c}}$. For the scalar-pseudoscalar coupling, for example, we then obtain 


$$
\begin{aligned}
\partial_{t} \lambda_{(\sigma-\pi)}= & 2 \lambda_{(\sigma-\pi)}+32 N_{\mathrm{c}} v_{4}\left(-4 \lambda_{(\sigma-\pi)}^{2}-8 \lambda_{(\sigma-\pi)} \lambda_{(S+P)_{-}}-8 \lambda_{(S+P)_{-}}^{2}-2 \lambda_{(\sigma-\pi)} \lambda_{(S+P)_{-}^{\mathrm{adj}}-}-4 \lambda_{(S+P)_{-}} \lambda_{(S+P)_{-}^{\mathrm{adj}}}+\lambda_{(\sigma-\pi)} \lambda_{(V+A)_{\|}^{\mathrm{adj}}}+2 \lambda_{(\sigma-\pi)} \lambda_{\mathrm{csc}}\right. \\
& \left.+4 \lambda_{(S+P)_{-}} \lambda_{\mathrm{csc}}+2 \lambda_{(S+P)_{-}^{\mathrm{adj}}} \lambda_{\mathrm{csc}}\right) l_{\|+}^{\mathrm{F})}\left(\tau, 0,-i \tilde{\mu}_{\tau}\right) \\
& +16 N_{\mathrm{c}} v_{4}\left(-8 \lambda_{(\sigma-\pi)}^{2}-16 \lambda_{(\sigma-\pi)} \lambda_{(S+P)_{-}}-16 \lambda_{(S+P)_{-}}^{2}-4 \lambda_{(\sigma-\pi)} \lambda_{(S+P)_{-}^{\mathrm{adj}}}-8 \lambda_{(S+P)_{-}} \lambda_{(S+P)_{-}^{\mathrm{adj}}}-\frac{4}{3} \lambda_{(S+P)_{-}^{\mathrm{adj}}}^{2}\right. \\
& \left.+2 \lambda_{(\sigma-\pi)} \lambda_{(V+A)_{\|}^{\mathrm{adj}}}-\frac{1}{3} \lambda_{(V+A)_{\|}^{\mathrm{adj}}}^{2}+4 \lambda_{(\sigma-\pi)} \lambda_{\mathrm{csc}}+8 \lambda_{(S+P)_{-}} \lambda_{\mathrm{csc}}+\frac{4}{3} \lambda_{(S+P)_{-}^{\mathrm{adj}}} \lambda_{\mathrm{csc}}-\frac{4}{3} \lambda_{\mathrm{csc}}^{2}\right) l_{\perp+}^{\mathrm{F})}\left(\tau, 0,-i \tilde{\mu}_{\tau}\right)
\end{aligned}
$$

For the remaining nine four-quark couplings, we find that the right-hand sides of their flow equations do not contain terms quadratic in the scalar-pseudoscalar coupling $\lambda_{(\sigma-\pi)}$ but at most terms linear in $\lambda_{(\sigma-\pi)}$ in the large- $N_{\mathrm{c}}$ limit. At first glance, this may not appear noteworthy. However, by setting all four-quark couplings but the scalar-pseudoscalar coupling to zero on the right-hand sides of the flow equations, we therefore observe that only the right-hand side of the flow equation of the scalar-pseudoscalar coupling remains finite. Indeed, from Eq. (35), we deduce that

$$
\begin{aligned}
\partial_{t} \lambda_{(\sigma-\pi)}= & 2 \lambda_{(\sigma-\pi)}-128 N_{\mathrm{c}} v_{4} \lambda_{(\sigma-\pi)}^{2}\left(l_{\|+}^{(\mathrm{F})}\left(\tau, 0,-i \tilde{\mu}_{\tau}\right)\right. \\
& \left.+l_{\perp+}^{(\mathrm{F})}\left(\tau, 0,-i \tilde{\mu}_{\tau}\right)\right) .
\end{aligned}
$$

Note that this flow equation is identical to Eq. (28) in the large- $N_{\mathrm{c}}$ limit.

The right-hand sides of the flow equations of the remaining nine couplings are identical to zero when we set all four-quark couplings but the scalar-pseudoscalar coupling to zero in the large- $N_{\mathrm{c}}$ limit. Thus, we have found a nontrivial fixed point of the RG flow at

$$
\lambda_{(\sigma-\pi)}^{*}=\frac{2 \pi^{2}}{N_{\mathrm{c}}} \quad \text { and } \quad \lambda_{j}^{*}=0
$$

which "sits" on the pure scalar-pseudoscalar axis of our tendimensional space spanned by the four-quark couplings. Here, we have $j \in \mathcal{B}$ but $j \neq(\sigma-\pi)$, and $\mathcal{B}$ denotes the set of indices associated with our Fierz-complete basis of fourquark interactions.

The fixed point (37) has only one IR repulsive direction, namely, the one associated with the scalar-pseudoscalar axis. The remaining nine directions are all IR attractive. This observation already suggests that the scalar-pseudoscalar channel dominates the low-energy dynamics, provided that we initiate the RG flow sufficiently close to this fixed point. ${ }^{14}$ We add that the dynamics of our Fierzcomplete system is governed by $2^{10}=1024$ fixed points. Depending on the temperature and the quark chemical

\footnotetext{
${ }^{14} \mathrm{We}$ do not aim at a precise determination of the size of the associated domain of attraction.
}

potential, some of these fixed points appear in complexconjugated pairs, as we shall discuss in Sec. IV E.

We emphasize that, for any finite value of $N_{\mathrm{c}}$, we do not find an interacting fixed point on the pure scalar-pseudoscalar axis. ${ }^{15}$ In fact, not only does the flow equation of the scalar-pseudoscalar coupling contain terms proportional to the square of the scalar-pseudoscalar coupling, but they also appear in the flow equations of other four-quark couplings. These terms now dynamically generate interactions in channels other than the scalar-pseudoscalar channel, pushing the fixed point (37) away from the scalar-pseudoscalar axis. We add that the very same behavior has also been observed in the vacuum limit of the $U_{\mathrm{A}}(1)$-symmetric NJL model in the large- $N_{\mathrm{c}}$ limit [8] and the three-dimensional Thirring model in the large- $N_{\mathrm{f}}$ limit [52].

The existence of the fixed point (37) and its properties provides us with an explanation of the phase structure at small quark chemical potential. First of all, from the standpoint of model studies, the existence of this fixed point in the large- $N_{\mathrm{c}}$ limit implies that the system always remains on the scalar-pseudoscalar axis, provided that we only choose a finite initial value for the scalar-pseudoscalar coupling and set all the other couplings to zero. Thus, the other channels do not contribute at all. Given the scalefixing procedure underlying our calculations, it then follows that the phase boundary found in the scalarpseudoscalar one-channel approximation agrees identically with the one from the Fierz-complete study in the large- $N_{\mathrm{c}}$ limit. ${ }^{16}$

Beyond the large- $N_{\mathrm{c}}$ limit, the fixed point (37) is pushed away from the scalar-pseudoscalar axis, and now all fourquark interactions are generated dynamically even if only the scalar-pseudoscalar coupling is chosen to be finite at the initial RG scale; see, e.g., Fig. 3 and also our discussion in Sec. IV E below. However, the observed agreement of the results for the phase boundary from the one-channel and the

\footnotetext{
${ }^{15}$ The scalar-pseudoscalar axis may be viewed as the axis associated with conventional NJL model studies taking into account only this channel.

${ }^{16}$ Note that, strictly speaking, invariance under Fierz transformations is violated in the large- $N_{\mathrm{c}}$ limit.
} 
Fierz-complete study suggests that this fixed point still controls the dynamics of the theory at small quark chemical potential; see Fig. 2. Even nonuniversal quantities such as the curvature of the phase boundary at $\mu=0$ appear to be independent of the inclusion of the dynamics described by the channels other than the scalar-pseudoscalar channel. Only for large values of the quark chemical potential, $\mu>\mu_{\chi}$, the influence of the other channels becomes significant. Recall that, in the mean-field approximation, $\mu_{\chi}$ is of the order of the vacuum constituent quark mass.

A word of caution needs to be added to this intriguing observation: if we choose initial conditions such that not only the scalar-pseudoscalar coupling is finite at the initial RG scale but also other four-quark couplings are, then the RG flow may be potentially controlled by a different interacting fixed point, even at small chemical potential. As a consequence, the phase boundary in this regime may become more sensitive to the dynamics described by the full set of four-quark interactions. For example, one may choose a $U_{\mathrm{A}}(1)$-symmetric starting point of the RG flow by tuning the couplings $\bar{\lambda}_{(\sigma-\pi)}$ and $\bar{\lambda}_{(S+P)_{-}}$such that the sum rule (18) is fulfilled. However, even in this case, we observe that, at small $\mu$, the phase boundary obtained from a Fierz-complete $U_{\mathrm{A}}(1)$-symmetric study agrees very well with the one from our onechannel approximation as well as with the one from our Fierz-complete study taking $U_{\mathrm{A}}(1)$-symmetry breaking into account; see, e.g., Fig. 6.

\section{E. Symmetry breaking mechanisms}

Let us finally analyze the mechanisms underlying the phase structure at large chemical potential where corrections beyond the large- $N_{\mathrm{c}}$ approximation become important. Looking at the modulus of the four-quark couplings depicted in Fig. 4, we observe that the scalarpseudoscalar coupling and the CSC coupling are the two most dominant couplings in the range of quark chemical potentials studied in this work, at least close to and above the phase transition line. For an analysis of the symmetry breaking mechanisms, it therefore appears reasonable to consider an approximation which only includes the scalar-pseudoscalar coupling and the CSC coupling. The remaining eight couplings and their flows are set to zero. The flow equations of such a two-channel approximation then read

$$
\begin{gathered}
\partial_{t} \lambda_{(\sigma-\pi)}=2 \lambda_{(\sigma-\pi)}+64 v_{4}\left(-\left(2 N_{\mathrm{c}}+1\right) \lambda_{(\sigma-\pi)}^{2}+\left(N_{\mathrm{c}}+1\right) \lambda_{(\sigma-\pi)} \lambda_{\mathrm{csc}}\right) l_{\|+}^{(\mathrm{F})}\left(\tau, 0,-i \mu_{\tau}\right) \\
+64 v_{4}\left(-\left(2 N_{\mathrm{c}}+1\right) \lambda_{(\sigma-\pi)}^{2}+\frac{1}{3}\left(3 N_{\mathrm{c}}-1\right) \lambda_{(\sigma-\pi)} \lambda_{\mathrm{csc}}-\frac{1}{3}\left(N_{\mathrm{c}}-2\right) \lambda_{\mathrm{csc}}^{2}\right) l_{\perp+}^{(\mathrm{F})}\left(\tau, 0,-i \mu_{\tau}\right), \\
\partial_{t} \lambda_{\mathrm{csc}}=2 \lambda_{\mathrm{csc}}+64 v_{4}\left(-\lambda_{(\sigma-\pi)}^{2}+\left(N_{\mathrm{c}}-2\right) \lambda_{\mathrm{csc}}^{2}\right) l_{\|+}^{(\mathrm{F})}\left(\tau, 0,-i \mu_{\tau}\right)+64 v_{4}\left(-\lambda_{(\sigma-\pi)}^{2}-2 \lambda_{(\sigma-\pi)} \lambda_{\mathrm{csc}}+4 \lambda_{\mathrm{csc}}^{2}\right) l_{\| \pm}^{(\mathrm{F})}\left(\tau, 0,-i \mu_{\tau}\right) \\
+64 \lambda_{(\sigma-\pi)}^{2} v_{4} l_{\perp+}^{(\mathrm{F})}\left(\tau, 0,-i \mu_{\tau}\right)+64 v_{4}\left(\lambda_{(\sigma-\pi)}^{2}-2 \lambda_{(\sigma-\pi)} \lambda_{\mathrm{csc}}+4 \lambda_{\mathrm{csc}}^{2}\right) l_{\perp \pm}^{\mathrm{F})}\left(\tau, 0,-i \mu_{\tau}\right) .
\end{gathered}
$$

The initial conditions are chosen as in our Fierzcomplete study; i.e., we set the CSC coupling to zero at the initial RG scale and only tune the scalar-pseudoscalar coupling such that the value for the symmetry breaking scale in the vacuum limit is identical to its value in the Fierz-complete study, $k_{0}=k_{\mathrm{cr}}(T=0, \mu=0)$, which, in turn, is identical to the value of the critical scale in our mean-field approximation. From the set of the two flow equations (38) and (39), we immediately deduce that the CSC coupling is dynamically generated in the RG flow, although we set it to zero at the initial RG scale.

An asset of our two-channel approximation is that it allows for a comparatively simple but still detailed analysis of the RG flow of our system and its fixed-point structure. Of course, such an analysis is also possible for more than two couplings, but it then clearly becomes more involved. In any case, in our two-channel approximation, we have four fixed points $\mathcal{F}_{j}=\left(\lambda_{(\sigma-\pi), j}^{*}, \lambda_{\mathrm{csc}, j}^{*}\right)$ in total. At $T=0$, $\mu=0$, and $N_{\mathrm{c}}=3$, their coordinates are

$$
\begin{gathered}
\left.\mathcal{F}_{1}\right|_{N_{\mathrm{c}}=3}=(0,0), \\
\left.\mathcal{F}_{2}\right|_{N_{\mathrm{c}}=3} \approx(5.165,-1.088), \\
\left.\mathcal{F}_{3}\right|_{N_{\mathrm{c}}=3} \approx(1.262-\mathrm{i} 1.567,-8.728-\mathrm{i} 0.841), \\
\left.\mathcal{F}_{4}\right|_{N_{\mathrm{c}}=3} \approx(1.262+\mathrm{i} 1.567,-8.728+\mathrm{i} 0.841),
\end{gathered}
$$

where $\mathcal{F}_{1}$ is the $N_{\mathrm{c}}$-independent Gaussian fixed point with two IR attractive directions. Apparently, $\mathcal{F}_{3}$ and $\mathcal{F}_{4}$ form a pair of complex-conjugate fixed points. The coordinates of the non-Gaussian fixed points up to order $1 / N_{\mathrm{c}}^{2}$ in a large$N_{\mathrm{c}}$ expansion read 


$$
\begin{aligned}
\mathcal{F}_{2}\left(N_{\mathrm{c}}\right)= & \left(\frac{2 \pi^{2}}{N_{\mathrm{c}}}-\frac{3 \pi^{2}}{2 N_{\mathrm{c}}^{2}},-\frac{\pi^{2}}{N_{\mathrm{c}}^{2}}\right), \\
\mathcal{F}_{3}\left(N_{\mathrm{c}}\right)= & \left(-\frac{(3+\mathrm{i} \sqrt{23}) \pi^{2}}{N_{\mathrm{c}}}+\frac{(235 \sqrt{10}+\mathrm{i} 6 \sqrt{11481}) \pi^{2}}{4 \sqrt{10} N_{\mathrm{c}}^{2}},\right. \\
& \left.-\frac{16 \pi^{2}}{N_{\mathrm{c}}}+\frac{(393-\mathrm{i} 13 \sqrt{23}) \pi^{2}}{2 N_{\mathrm{c}}^{2}}\right), \\
\mathcal{F}_{4}\left(N_{\mathrm{c}}\right)= & \left(-\frac{(3-\mathrm{i} \sqrt{23}) \pi^{2}}{N_{\mathrm{c}}}+\frac{(235 \sqrt{10}+\mathrm{i} 6 \sqrt{11481}) \pi^{2}}{4 \sqrt{10} N_{\mathrm{c}}^{2}},\right. \\
& \left.-\frac{16 \pi^{2}}{N_{\mathrm{c}}}+\frac{(393+\mathrm{i} 13 \sqrt{23}) \pi^{2}}{2 N_{\mathrm{c}}^{2}}\right) .
\end{aligned}
$$

These expansions have been extracted from the full analytic $N_{\mathrm{c}}$-dependent expressions for the coordinates of the fixed points. We observe that the suitably $N_{\mathrm{c}^{-}}$ rescaled fixed point $N_{\mathrm{c}} \cdot \mathcal{F}_{2}$ is shifted onto the scalarpseudoscalar axis for $N_{\mathrm{c}} \rightarrow \infty$. Moreover, we find that this fixed point has one IR repulsive and one IR attractive direction. Thus, this fixed point corresponds to the fixed point (37) in the full Fierz-complete set of RG flow equations.

In the following, we shall not consider the large- $N_{\mathrm{c}}$ limit any further. To have spontaneous symmetry breaking in the IR limit, we then choose the initial condition of the scalarpseudoscalar coupling to be greater than $\lambda_{(\sigma-\pi), 2}^{*}$ but still set the initial value of the CSC coupling to zero; see our discussion above. As a consequence, we also find for this two-channel approximation that the low-energy dynamics is dominated by the scalar-pseudoscalar channel. The RG flow of this two-channel approximation is depicted in Fig. 7.

Next, let us discuss symmetry restoration at finite temperature and quark chemical potential with the aid of our two-channel approximation. The fixed points now become pseudo-fixed-points due to the presence of a dimensionful external parameter, namely, the temperature. ${ }^{17}$ As a consequence, the position of the nonGaussian fixed points is shifted as a function of the dimensionless temperature $T / k$, and therefore also the positions of the separatrices connecting the fixed points are shifted. This is illustrated in Fig. 8 for the RG flow in the plane spanned by the scalar-pseudoscalar and the CSC coupling at $T / k=0.4$ and $\mu=0$. While the fixed points $\mathcal{F}_{3}$ and $\mathcal{F}_{4}$ remain complex valued when $T / k$ is increased, the behavior of the real-valued non-Gaussian (pseudo-)fixed points suggests that, for initial conditions chosen to be fixed in the domain $\mathcal{D}_{1}$ (see, e.g., the pink dot in Fig. 8), a critical temperature $T_{\text {cr }}$ exists above which the four-quark couplings do not diverge anymore at a finite RG scale $k_{\mathrm{cr}}$ but remain finite on all scales and approach zero in the IR limit, $k \rightarrow 0$. In other words,

\footnotetext{
${ }^{17}$ The same holds true in the case of a finite chemical potential.
}

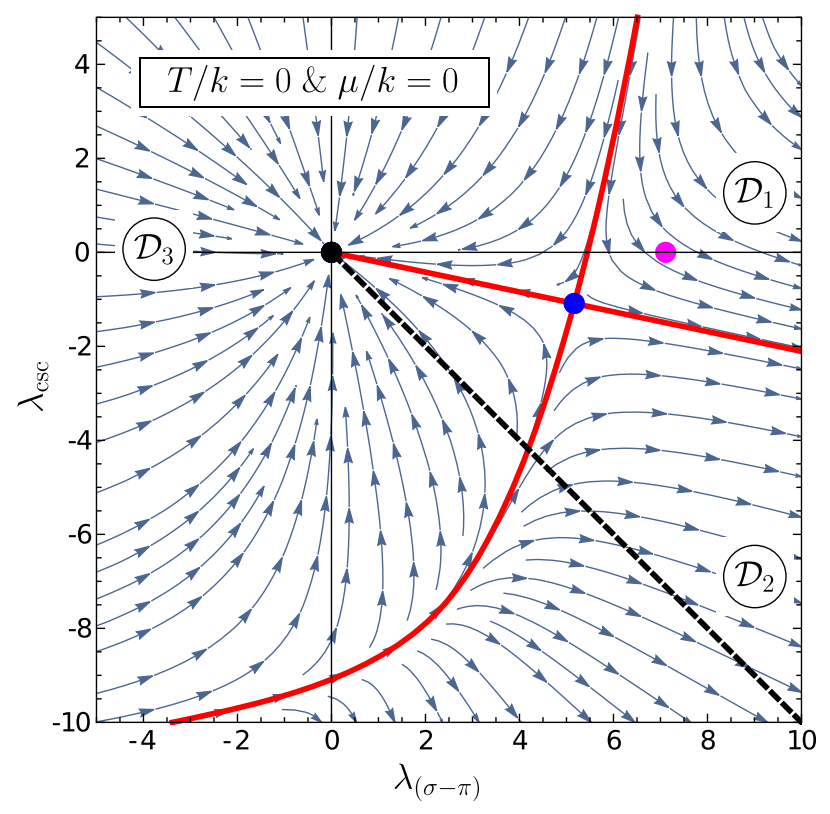

FIG. 7. RG flow of the two-channel approximation at zero temperature and chemical potential in the plane spanned by the scalar-pseudoscalar coupling and the CSC coupling. The black dot represents the Gaussian fixed point, whereas the blue dot represents the real-valued non-Gaussian fixed point; see Eq. (41). The pink dot depicts our choice for the initial condition. The RG trajectory starting at this point describes four-quark couplings diverging at a finite scale $k_{0}=k_{\mathrm{cr}}$, while approaching a separatrix (red solid line) as indicated by the arrows. The dominance of the scalar-pseudoscalar channel is illustrated by the position of the corresponding separatrix relative to the bisectrix (dashed back line). The different domains separated by the separatrices (red solid lines) are labeled $\mathcal{D}_{1}, \mathcal{D}_{2}$, and $\mathcal{D}_{3}$.

there is no (spontaneous) symmetry breaking above the critical temperature. At least at high temperature, such a behavior is indeed expected since the quarks become effectively stiff d.o.f. due to their thermal Matsubara mass $\sim T$. This mechanism has already been discussed in detail in Refs. $[8,10]$ and underlies symmetry restoration when the temperature is increased.

Before we continue with a discussion of the mechanisms underlying symmetry breaking at zero temperature and finite quark chemical potential, we would like to comment on the curvature of the finite-temperature phase boundary at small $\mu$. We observe that the curvature extracted from our two-channel approximation agrees almost identically with the curvatures found in the one-channel approximation as well as in the Fierz-complete study. The agreement of the latter two can be understood in terms of the fixed-point structure as discussed above. Of course, the agreement of the curvatures obtained from the two-channel approximation and the Fierz-complete study can also be understood from their fixed-point structure. However, we would like to 


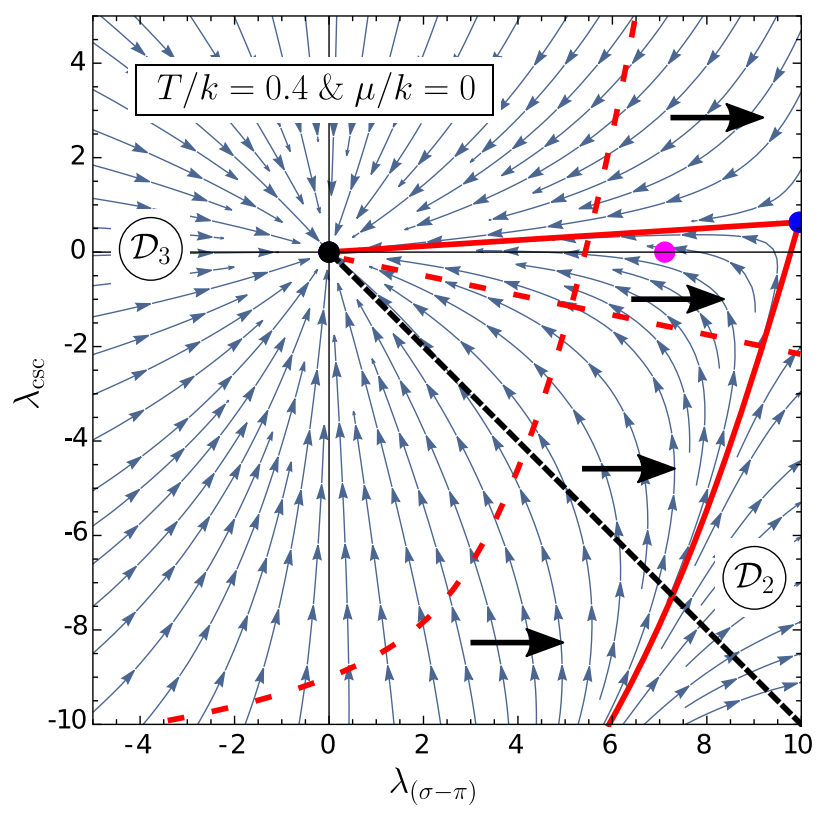

FIG. 8. RG flow of the two-channel approximation at $T / k=$ 0.4 and $\mu=0$ in the plane spanned by the scalar-pseudoscalar coupling and the CSC coupling. The black dot represents the Gaussian fixed point. The blue dot represents the real-valued non-Gaussian fixed point. The pink dot depicts our choice for the initial condition. The dashed black line is the bisectrix of the bottom right quadrant. The different domains separated by the separatrices (red solid lines) are labeled $\mathcal{D}_{2}$, and $\mathcal{D}_{3}$; see also Fig. 7. $\mathcal{D}_{1}$ is not shown. The black arrows indicate the shift of the real-valued non-Gaussian fixed point together with the domains $\mathcal{D}_{1}$ and $\mathcal{D}_{2}$ when $T / k$ is increased; see the main text for details. The dashed red lines depict the position of the separatrices in the vacuum limit; see also Fig. 7.

recall that the flow equations of the two-channel approximation suffer from the Fierz ambiguity. Our two-channel approximation has been extracted from the Fierz-complete set of equations by only taking into account the scalar-pseudoscalar coupling and the CSC coupling. The remaining eight couplings and their flows have been set to zero. This is well justified for the purpose of analyzing the mechanisms underlying the structure of the phase diagram found in the Fierzcomplete study. In practice, however, the flow equations for a two-channel approximation are not obtained from the full Fierz-complete set but by rather only taking into account the scalar-pseudoscalar channel and the CSC channel in our Ansatz for the effective action (6). Owing to the freedom of performing Fierz transformations, the set of flow equations for these two couplings resulting from such a Fierz-incomplete Ansatz will differ from the one used in our present analysis. For example, terms associated with a Feynman diagram of the type depicted in the right panel of Fig. 1 may be found to contribute to the flow of the scalar-pseudoscalar coupling. As we have seen in our discussion of the large- $N_{\mathrm{c}}$ limit, such contributions are parametrically suppressed by factors of $1 / N_{\mathrm{c}}$ compared to those associated with Feynman diagrams of the type shown in the left panel of Fig. 1. Thus, these contributions drop out for $N_{\mathrm{c}} \rightarrow \infty$. For finite $N_{\mathrm{c}}$, however, they may still alter the curvature significantly; see Ref. [10].

Let us now turn to the discussion of the dense regime of the phase diagram. At zero temperature, we do not observe symmetry restoration in our Fierz-complete study when the quark chemical potential is increased; see Fig. 2. As can also be seen in Fig. 2, the same behavior is found in our present two-channel approximation. Even though we do not observe symmetry restoration at zero temperature when the chemical potential $\mu$ is increased, we find that the hierarchy of the channels changes as a function of $\mu$; i.e., the CSC channel becomes the most dominant channel for sufficiently large values of the chemical potential, $\mu \gtrsim \mu_{\chi}$. Note that a dominance of the CSC channel is associated with a divergence of the RG flow into the direction defined by the CSC coupling. Given our choice for the initial condition (see the pink dot in Figs. 7, 8, and 9), such a dominance is not immediately apparent. In fact, even if we chose the initial condition to be located in the domain $\mathcal{D}_{2}$, we would still observe a dominance of the scalar-pseudoscalar channel at low energies. Thus, a dominance of the CSC channel is prohibited by the vacuum fixed-point structure. This can be traced back to the fact that the fixed point $\mathcal{F}_{2}$ has one IR attractive and one IR repulsive direction. ${ }^{18}$ Increasing the quark chemical potential starting from the vacuum limit, we find that the fixed-point structure together with the position of the separatrices remains unchanged up to a "critical value" $(\mu / k)_{0}$ of the dimensionless chemical potential. ${ }^{19}$ At $\mu / k=(\mu / k)_{0}$, we then observe that two new real-valued fixed points emerge in the plane spanned by the scalar-pseudoscalar coupling and the CSC coupling, which sit on top of each other; see the top right panel of Fig. 9. These two "new" fixed points are nothing but the fixed points $\mathcal{F}_{3}$ and $\mathcal{F}_{4}$, which become real valued at $\mu / k=(\mu / k)_{0}$. As a consequence of this "creation" of two real-valued fixed points, new separatrices emerge in the plane spanned by the two four-quark couplings, which divide the parameter space into five domains $\mathcal{D}_{i}$; see, e.g., the top right panel of Fig. 9. Still, an RG trajectory associated with a dominance of the CSC coupling cannot be established for initial conditions located in the domain $\mathcal{D}_{1}$. Increasing $\mu / k$ further, the two new

\footnotetext{
${ }^{18}$ Recall that the fixed point $\mathcal{F}_{2}$ corresponds to the fixed point (37) in the Fierz-complete study.

${ }^{19}$ We observe slight changes of the fixed-point structure and the associated positions of the separatrices for $\mu / k \lesssim(\mu / k)_{0}$, which arise due to a mild violation of the Silver-Blaze property by our covariant regularization scheme; see Ref. [10] for a detailed discussion of this aspect.
} 

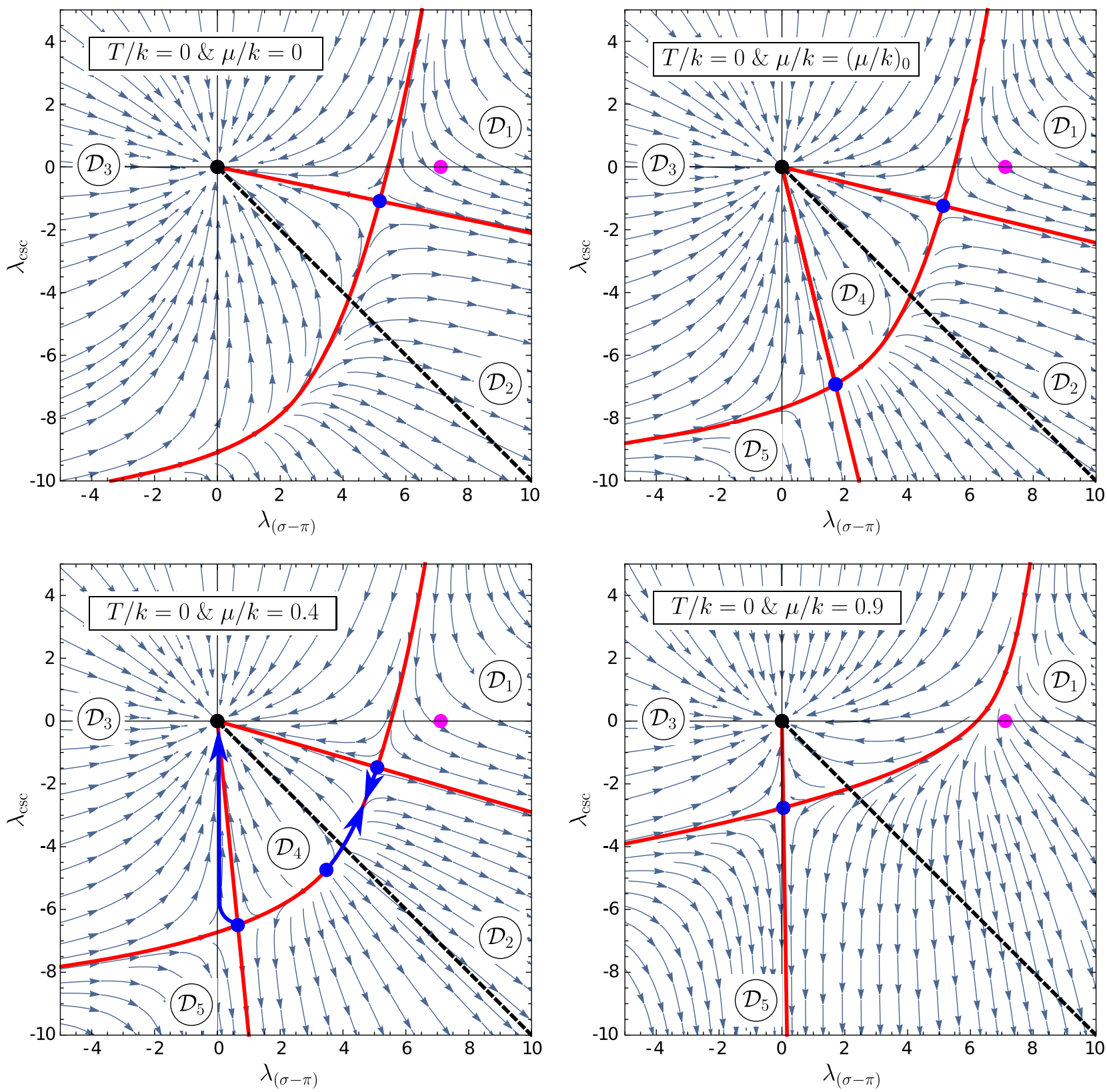

FIG. 9. RG flow of the two-channel approximation at $T=0$ for different values of $\mu / k: \mu / k=0$ (top left panel; same as Fig. 7), $\mu / k=(\mu / k)_{0} \approx 0.298$ (top right panel), $\mu / k=0.4$ (bottom left panel), and $\mu / k=0.9$ (bottom right panel) in the plane spanned by the scalar-pseudoscalar coupling and the CSC coupling. The black dot represents the Gaussian fixed point. Blue dots represent real-valued non-Gaussian fixed points. The pink dot depicts our choice for the initial condition. The dashed black line is the bisectrix of the bottom right quadrant. Different domains $\mathcal{D}_{i}$ are separated by separatrices (red solid lines). The blue arrows in the bottom left panel indicate the shift of the real-valued non-Gaussian fixed points when $\mu / k$ is increased; see the main text for details.

real-valued non-Gaussian (pseudo-)fixed points are shifted in different directions as indicated by the blue arrows in the bottom left panel of Fig. 9. One of these two fixed points has one attractive and one repulsive direction and is shifted toward the Gaussian fixed point. The other one is shifted toward the fixed point $\mathcal{F}_{2}$. At sufficiently large $\mu / k>(\mu / k)_{0}$, the latter two then annihilate each other in the sense that they become complex-valued fixed points.
This annihilation also removes the separatrix separating the domains $\mathcal{D}_{1}$ and $\mathcal{D}_{2}$. As a consequence, any initial condition of the RG flow located in the domain $\mathcal{D}_{1}$ now yields an RG trajectory eventually pointing into the direction associated with the CSC coupling with the two couplings diverging at a finite critical scale $k_{\mathrm{cr}}$. In other words, for sufficiently large $\mu / k$, the low-energy physics is potentially dominated by the dynamics associated 
with the CSC channel. ${ }^{20}$ The remaining real-valued fixed point at large $\mu / k$ is eventually shifted toward the Gaussian fixed point for $\mu / k \rightarrow \infty$. As has been shown in Ref. [10], the merging of the latter two fixed points is associated with the Cooper instability. Indeed, this behavior leaves its imprint in the $\mu$ dependence of the symmetry breaking scale, exhibiting the typical Bardeen-Cooper-Schrieffer-type exponential scaling behavior.

\section{CONCLUSIONS}

In this work, we have used RG flow equations of fourquark couplings to analyze the phase structure of an NJLtype model with two quark flavors coming in $N_{\mathrm{c}}$ colors at leading order of the derivative expansion of the effective action. With our study, we aimed at an understanding how Fierz-incomplete approximations affect the predictive power of this class of models, which still underlies to a large extent our understanding of the dynamics of QCD at high density. Our present leading-order approximation of the effective action already includes corrections beyond the often-employed mean-field approximation. Note that such corrections are ultimately required to preserve the invariance of the results under Fierz transformations [48].

Our results suggest that Fierz incompleteness strongly affects the phase structure. For example, the phase transition temperature at large chemical potential almost increases by a factor of 2 compared to a study which only includes the conventional scalar-pseudoscalar interaction channel together with a channel associated with the formation of a color-superconducting ground state. Although we do not have direct access to the gap within our present study, the observed shift of the phase boundary may suggest that the use of Fierz-incomplete approximations also affects the magnitude of the gap in the high-density regime. This is in accord with mean-field studies of this regime (see, e.g., Refs. [3-7] for reviews). However, we rush to add that the strength of the effect is expected to depend on the details of the chosen basis of four-quark interactions and the actual choice for the initial conditions of the RG flow equations, i.e., the choice for the parameters appearing in the classical action.

Despite the fact that our present study relies on a Fierzcomplete approximation, it is clear that our results are mostly qualitative. In fact, our present study based on the analysis of RG flow equations of four-quark interactions at leading order of the derivative expansion is limited with respect to a determination of the properties of the actual ground state

\footnotetext{
${ }^{20}$ Note that, as we solve the RG flow from $k=\Lambda$ to $k \rightarrow 0$, the dimensionless chemical potential $\mu / k$ changes from $\mu / \Lambda \gtrsim 0$ to $\mu / k \rightarrow \infty$. In terms of the RG time $t=\ln (k / \Lambda)$, however, the four-quark couplings may already diverge at a finite value of $k$ before the RG flow fully changes its direction at a certain value of $\mu / k$. Large values of $\mu / k$ may therefore not be reached in the RG flow, and the scalar-pseudoscalar channel may still dominate the dynamics at sufficiently small values of $\mu$.
}

in the phase governed by spontaneous symmetry breaking. To gain at least some insight into the structure of the ground state, we have analyzed the hierarchy of the four-quark interactions (in terms of their strength) as a function of the temperature and the quark chemical potential, following the analysis in Ref. [10]. A dominance of a given channel may then be considered as an indication for the formation of a corresponding condensate. Of course, such an analysis has to be taken with some care as a dominance of a given channel may not necessarily entail condensation in this channel. Moreover, more than one condensate may be formed, e.g., at large chemical potential. Still, it allows us to gain some insight into the dynamics underlying the phase structure. Interestingly, in our present Fierz-complete study, we observe that the dynamics close to the phase boundary at small quark chemical potential is clearly dominated by the scalar-pseudoscalar interaction channel, whereas the channel associated with the formation of the most conventional color-superconducting condensate dominates the dynamics at large chemical potential. In the latter regime, the scalar-pseudoscalar channel is found to be only subdominant. Even more, the channels associated with the formation of color-symmetry breaking condensates are most dominant in this regime.

To understand better the dynamics underlying the phase structure, we have analyzed our Fierz-complete study in several ways. For example, we have monitored the strength of $U_{\mathrm{A}}(1)$ symmetry breaking and even studied a $U_{\mathrm{A}}(1)$ symmetric variation of our model which indicated that the hierarchy of the channels changes at large chemical potential in this case. Moreover, we considered our flow equation in the large- $N_{\mathrm{c}}$ limit, which revealed the existence of a fixed point which controls the dynamics at least for small values of the chemical potential, provided the initial conditions have been chosen to be located in a specific domain in the space spanned by our set of four-quark couplings. At large chemical potential, the leading order of the large- $N_{\mathrm{c}}$ expansion cannot be used to explain the phase structure since channels subleading in this expansion become important. With the aid of a suitably chosen two-channel approximation, however, we have found that the phase structure and the dominance of the color-superconducting channel at large chemical potential is a consequence of an intriguing creation and annihilation of pairs of (pseudo-)fixed points.

Finally, we emphasize again that, at the present order of the derivative expansion, our analysis is still qualitative regarding the determination of the actual properties of the ground state. To unambiguously determine the ground-state properties, a calculation of the full at least ten-dimensional order-parameter potential would in principle be required, representing an ambitious continuation of, e.g., recent beyond-mean field calculations of the order-parameter potential with a scalarpseudoscalar and a diquark channel $[31,53]$ as well as with a scalar-pseudoscalar and a vector channel [54]. Nevertheless, our present analysis already provides new insight into the phase structure and the ground-state properties of NJL-type models at finite temperature and density and may therefore 
also be helpful for future studies of bulk quantities, such as the equation of state at high density.

\section{ACKNOWLEDGMENTS}

The authors would like to thank H. Gies and J. M. Pawlowski for useful discussions and comments on the manuscript. As members of the fQCD Collaboration [55], the authors also would like to thank the other members of this collaboration for discussions. J. B. acknowledges support by HIC for FAIR within the LOEWE program of the State of Hesse. This work is supported by the Deutsche Forschungsgemeinschaft (DFG) through Grant No. SFB 1245.

\section{APPENDIX A: RG FORMALISM}

For the computation of the RG flow equations of the various couplings and renormalization factors, we have employed the Wetterich equation [34], which represents an $\mathrm{RG}$ equation for the (scale-dependent) quantum effective action $\Gamma_{k}$ :

$$
\partial_{t} \Gamma_{k}[\Phi]=-\frac{1}{2} \operatorname{Tr}\left\{\left[\Gamma_{k}^{(1,1)}[\Phi]+R_{k}^{\psi}\right]^{-1} \cdot\left(\partial_{t} R_{k}^{\psi}\right)\right\} .
$$

Here, $\Gamma_{k}^{(1,1)}$ denotes the second functional derivative of the (scale-dependent) quantum effective action $\Gamma_{k}$ with respect to the fermion fields summarized in the field vector $\Phi^{T}(q)=\left(\psi^{T}(q), \bar{\psi}(-q)\right)$. For an explicit calculation of $\mathrm{RG}$ flow equations, we have to specify the regulator function $R_{k}^{\psi}$, which encodes the regularization scheme. In this work, we have employed a four-dimensional so-called Fermi-surface-adapted regulator function as introduced in Ref. [10]: $R_{k}^{\psi \prime}=-\left(\not p+\mathrm{i} \gamma_{0} \mu\right) r_{\psi}$. Here, the regulator shape function $r_{\psi}$ is defined as

$$
r_{\psi}=\frac{1}{\sqrt{1-\mathrm{e}^{-\bar{\omega}_{+} \bar{\omega}_{-}}}}-1,
$$

where $\bar{\omega}_{ \pm}=\omega_{ \pm} / k$ and $\omega_{ \pm}^{2}=p_{0}^{2}+(|\vec{p}| \pm \mu)^{2}$; see Ref. [10] for a detailed discussion of the properties of this regulator.

\section{APPENDIX B: RG FLOW EQUATIONS}

For an introduction to the derivation of RG flow equations of four-fermion interactions, we refer the reader to Ref. [8]. A general expression for the flow equations of pointlike four-fermion interactions of a general class of relativistic fermionic theories with a continuous chiral $U_{\mathrm{L}}\left(N_{\mathrm{f}}\right) \otimes U_{\mathrm{R}}\left(N_{\mathrm{f}}\right)$ symmetry in a Fierz-complete setting can be found in Ref. [56]. For the derivation of the flow equations of our present model with a $S U\left(N_{\mathrm{c}}\right) \otimes$ $S U_{\mathrm{L}}(2) \otimes S U_{\mathrm{R}}(2) \otimes U_{\mathrm{V}}(1)$ symmetry, we have made use of existing software packages [57,58]. In the following, we list the set of flow equations underlying our Fierzcomplete study for general $N_{\mathrm{c}}$ and $N_{\mathrm{f}}=2$ :

$$
\begin{aligned}
& \partial_{t} \lambda_{(\sigma-\pi)}=2 \lambda_{(\sigma-\pi)}+64 v_{4}\left(-\lambda_{(\sigma-\pi)}^{2}-4 \lambda_{(\sigma-\pi)} \lambda_{(S+P)_{-}}-4 \lambda_{(S+P)_{-}}^{2}+\lambda_{(\sigma-\pi)} \lambda_{(V+A)_{\|}}+\lambda_{(\sigma-\pi)} \lambda_{(V-A)_{\|}}+3 \lambda_{(\sigma-\pi)} \lambda_{(V+A)_{\perp}}\right.
\end{aligned}
$$

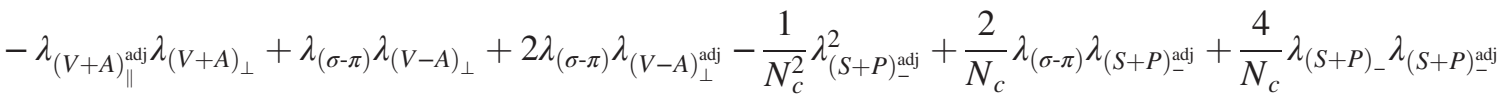

$$
\begin{aligned}
& +\frac{1}{N_{c}} \lambda_{(S+P)_{-}^{2}}^{2}{ }_{-j}-\frac{1}{2 N_{c}} \lambda_{(\sigma-\pi)} \lambda_{(V+A)_{\|}^{\mathrm{adj}}}-\frac{1}{2 N_{c}} \lambda_{(\sigma-\pi)} \lambda_{(V-A)_{\perp}^{\mathrm{adj}}}-2 N_{c} \lambda_{(\sigma-\pi)}^{2}-4 N_{c} \lambda_{(\sigma-\pi)} \lambda_{(S+P)_{-}}-4 N_{c} \lambda_{(S+P)_{-}}^{2} \\
& -N_{c} \lambda_{(\sigma-\pi)} \lambda_{(S+P)_{-}^{\mathrm{adj}}}-2 N_{c} \lambda_{(S+P)_{-}} \lambda_{(S+P)_{-}^{\mathrm{adj}}}+\frac{N_{c}}{2} \lambda_{(\sigma-\pi)} \lambda_{(V+A)_{\|}^{\mathrm{adj}}}+\lambda_{(\sigma-\pi)} \lambda_{\mathrm{csc}}-\lambda_{(S+P)_{-}^{\mathrm{ajj}}} \lambda_{\mathrm{csc}}+N_{c} \lambda_{(\sigma-\pi)} \lambda_{\mathrm{csc}} \\
& \left.+2 N_{c} \lambda_{(S+P)_{-}} \lambda_{\mathrm{csc}}+N_{c} \lambda_{(S+P)_{-}^{\mathrm{adj}}} \lambda_{\mathrm{csc}}\right) l_{\|+}^{(\mathrm{F})}\left(\tau, 0,-i \tilde{\mu}_{\tau}\right) \\
& +64 v_{4}\left(-\lambda_{(\sigma-\pi)} \lambda_{(V+A)_{\|}}+\lambda_{(\sigma-\pi)} \lambda_{(V+A)_{\perp}}+\lambda_{(V+A)_{\|}^{\mathrm{ajj}}} \lambda_{(V+A)_{\perp}}+\frac{1}{2 N_{c}} \lambda_{(\sigma-\pi)} \lambda_{(V+A)_{\|}^{\mathrm{ajj}}}\right) l_{\| \pm}^{(\mathrm{F})}\left(\tau, 0,-i \tilde{\mu}_{\tau}\right) \\
& +64 v_{4}\left(-\lambda_{(\sigma-\pi)}^{2}-4 \lambda_{(\sigma-\pi)} \lambda_{(S+P)_{-}}-4 \lambda_{(S+P)_{-}}^{2}-\frac{2}{3} \lambda_{(\sigma-\pi)} \lambda_{(S+P)_{-}^{\mathrm{ajj}_{-}}}-\frac{4}{3} \lambda_{(S+P)_{-}} \lambda_{(S+P)_{-}}{ }_{\mathrm{adj}}+\lambda_{(\sigma-\pi)} \lambda_{(V+A)_{\|}}+\frac{1}{3} \lambda_{(\sigma-\pi)} \lambda_{(V-A)_{\|}}\right. \\
& -\frac{1}{3} \lambda_{(V+A)_{\|}} \lambda_{(V+A)_{\|}^{\mathrm{adj}}}+3 \lambda_{(\sigma-\pi)} \lambda_{(V+A)_{\perp}}-\frac{2}{3} \lambda_{(V+A)_{\|}^{\mathrm{adj}}} \lambda_{(V+A)_{\perp}}+\frac{1}{3} \lambda_{(\sigma-\pi)} \lambda_{(V-A)_{\perp}}+\frac{2}{3} \lambda_{(\sigma-\pi)} \lambda_{(V-A)_{\perp}^{\mathrm{adj}}}-\frac{1}{N_{c}^{2}} \lambda_{(S+P)_{-}^{2}}^{2} \\
& +\frac{2}{N_{c}} \lambda_{(\sigma-\pi)} \lambda_{(S+P)_{-}^{\mathrm{adj}}}+\frac{4}{N_{c}} \lambda_{(S+P)_{-}} \lambda_{(S+P)_{-}^{\mathrm{adj}}}+\frac{5}{3 N_{c}} \lambda_{(S+P)_{-}^{\mathrm{adj}}}^{2}-\frac{1}{2 N_{c}} \lambda_{(\sigma-\pi)} \lambda_{(V+A)_{\|}^{\mathrm{adj}}}+\frac{1}{6 N_{c}} \lambda_{(V+A)_{\|}^{\mathrm{adj}}}^{2}-\frac{1}{6 N_{c}} \lambda_{(\sigma-\pi)} \lambda_{(V-A)_{\perp}^{\mathrm{adj}}} \\
& -2 N_{c} \lambda_{(\sigma-\pi)}^{2}-4 N_{c} \lambda_{(\sigma-\pi)} \lambda_{(S+P)_{-}}-4 N_{c} \lambda_{(S+P)_{-}}^{2}-N_{c} \lambda_{(\sigma-\pi)} \lambda_{(S+P)_{-}^{\mathrm{adj}}}-2 N_{c} \lambda_{(S+P)_{-}} \lambda_{(S+P)_{-}^{\mathrm{adj}}}-\frac{N_{c}}{3} \lambda_{(S+P)_{-}^{\mathrm{ajj}}}^{2}
\end{aligned}
$$




$$
\begin{aligned}
& +\frac{N_{c}}{2} \lambda_{(\sigma-\pi)} \lambda_{(V+A)_{\|}^{\mathrm{adj}}}-\frac{N_{c}}{12} \lambda_{(V+A)_{\|}^{\mathrm{adj}}}^{2}-\frac{1}{3} \lambda_{(\sigma-\pi)} \lambda_{\mathrm{csc}}-\frac{4}{3} \lambda_{(S+P)_{-}} \lambda_{\mathrm{csc}}-\frac{1}{3} \lambda_{(S+P)_{-}^{\mathrm{adj}}} \lambda_{\mathrm{csc}} \\
& \left.+\frac{2}{3 N_{c}} \lambda_{(S+P)_{-}^{\mathrm{adj}}} \lambda_{\mathrm{csc}}+N_{c} \lambda_{(\sigma-\pi)} \lambda_{\mathrm{csc}}+2 N_{c} \lambda_{(S+P)_{-}} \lambda_{\mathrm{csc}}+\frac{N_{c}}{3} \lambda_{(S+P)_{-}^{\mathrm{adj}}} \lambda_{\mathrm{csc}}+\frac{2}{3} \lambda_{\mathrm{csc}}^{2}-\frac{N_{c}}{3} \lambda_{\mathrm{csc}}^{2}\right) l_{\perp+}^{(\mathrm{F})}\left(\tau, 0,-i \tilde{\mu}_{\tau}\right) \\
& +64 v_{4}\left(\frac{1}{3} \lambda_{(\sigma-\pi)} \lambda_{(V+A)_{\|}}+\frac{1}{3} \lambda_{(V+A)_{\|}} \lambda_{(V+A)_{\|}^{\mathrm{adj}}}-\frac{5}{3} \lambda_{(\sigma-\pi)} \lambda_{(V+A)_{\perp}}-\frac{2}{3} \lambda_{(V+A)_{\|}^{\mathrm{adj}}} \lambda_{(V+A)_{\perp}}\right. \\
& -\frac{1}{6 N_{c}} \lambda_{(\sigma-\pi)} \lambda_{(V+A)_{\|}^{\mathrm{adj}}}-\frac{1}{6 N_{c}} \lambda_{(V+A)_{\|}^{\mathrm{adj}}}^{2} l_{\perp \pm}^{(\mathrm{F})}\left(\tau, 0,-i \tilde{\mu}_{\tau}\right), \\
& \partial_{t} \lambda_{\mathrm{csc}}=2 \lambda_{\mathrm{csc}}+64 v_{4}\left(-\lambda_{(\sigma-\pi)}^{2}+2 \lambda_{(\sigma-\pi)} \lambda_{(V+A)_{\|}^{\mathrm{adj}}}-\lambda_{(V+A)_{\|}^{\mathrm{adj}}}^{2}+3 \lambda_{(V-A)_{\perp}} \lambda_{(V-A)_{\perp}^{\mathrm{adj}}}-\frac{3}{2 N_{c}} \lambda_{(V-A)_{\perp}^{\mathrm{adj}}}^{2}+\frac{3 N_{c}}{4} \lambda_{(V-A)_{\perp}^{2}}^{\mathrm{adj}}\right. \\
& \left.+2 \lambda_{(V-A)} \lambda_{\mathrm{csc}}-\frac{3}{2} \lambda_{(V-A)_{\perp}^{\mathrm{adj}}} \lambda_{\mathrm{csc}}+\frac{3 N_{c}}{2} \lambda_{(V-A)_{\perp}^{\mathrm{ajj}}} \lambda_{\mathrm{csc}}-2 \lambda_{\mathrm{csc}}^{2}+N_{c} \lambda_{\mathrm{csc}}^{2}\right) l_{\|+}^{(\mathrm{F})}\left(\tau, 0,-i \tilde{\mu}_{\tau}\right) \\
& +64 v_{4}\left(-\lambda_{(\sigma-\pi)}^{2}-4 \lambda_{(\sigma-\pi)} \lambda_{(S+P)_{-}}-4 \lambda_{(S+P)_{-}}^{2}-4 \lambda_{(\sigma-\pi)} \lambda_{(S+P)_{-}^{\mathrm{adj}}}-8 \lambda_{(S+P)_{-}} \lambda_{(S+P)_{-}}^{\mathrm{adj}}-\lambda_{(S+P)_{-}^{\mathrm{adj}}}^{2}\right. \\
& -3 \lambda_{(V-A)_{\perp}} \lambda_{(V-A)_{\perp}^{\mathrm{adj}}}-\frac{1}{N_{c}^{2}} \lambda_{(S+P)_{-}^{\mathrm{adj}}}^{2}+\frac{2}{N_{c}} \lambda_{(\sigma-\pi)} \lambda_{(S+P)_{-}^{\mathrm{adj}}}+\frac{4}{N_{c}} \lambda_{(S+P)_{-}} \lambda_{(S+P)_{-}^{\mathrm{adj}}}+\frac{4}{N_{c}} \lambda_{(S+P)_{-}^{\mathrm{adj}}}^{2} \\
& +\frac{3}{2 N_{c}} \lambda_{(V-A)_{\perp}^{\mathrm{adj}}}^{2}-2 \lambda_{(\sigma-\pi)} \lambda_{\mathrm{csc}}-4 \lambda_{(S+P)_{-}} \lambda_{\mathrm{csc}}+2 \lambda_{\left.(S+P)_{-}\right)_{\mathrm{csc}}} \lambda_{\mathrm{csc}} \lambda_{(V-A)_{\|}} \lambda_{\mathrm{csc}}-3 \lambda_{(V-A)_{\perp}} \lambda_{\mathrm{csc}} \\
& \left.+\frac{3}{2} \lambda_{(V-A)_{\perp}^{\mathrm{adj}}} \lambda_{\mathrm{csc}}+\frac{2}{N_{c}} \lambda_{(S+P)_{-}^{\mathrm{adj}}} \lambda_{\mathrm{csc}}+\frac{3}{2 N_{c}} \lambda_{(V-A)_{\perp}^{\mathrm{adj}}} \lambda_{\mathrm{csc}}+4 \lambda_{\mathrm{csc}}^{2}\right) l_{\| \pm}^{(\mathrm{F})}\left(\tau, 0,-i \tilde{\mu}_{\tau}\right) \\
& +64 v_{4}\left(\lambda_{(\sigma-\pi)}^{2}-2 \lambda_{(\sigma-\pi)} \lambda_{(V+A)_{\|}^{\mathrm{adj}}}+\lambda_{(V+A)_{\|}^{\mathrm{adj}}}^{2}+\lambda_{(V-A)_{\|}} \lambda_{(V-A)_{\perp}^{\mathrm{adj}}}-2 \lambda_{(V-A)_{\perp}} \lambda_{(V-A)_{\perp}^{\mathrm{adj}}}+\frac{1}{N_{c}} \lambda_{(V-A)_{\perp}^{2}}^{\mathrm{adj}}-\frac{N_{c}}{2} \lambda_{(V-A)_{\perp}^{\mathrm{adj}}}^{2}\right.
\end{aligned}
$$

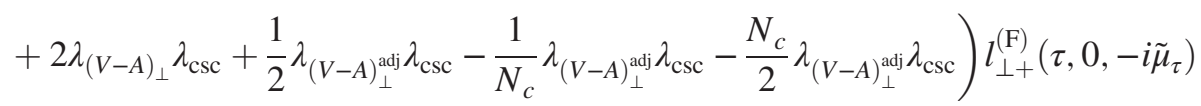

$$
\begin{aligned}
& +64 v_{4}\left(\lambda_{(\sigma-\pi)}^{2}+4 \lambda_{(\sigma-\pi)} \lambda_{(S+P)_{-}}+4 \lambda_{(S+P)_{-}}^{2}+\lambda_{(S+P)_{-}^{\mathrm{adj}}}^{2}-\lambda_{(V-A)_{\|}} \lambda_{(V-A)_{\perp}^{\mathrm{adj}}}-2 \lambda_{(V-A)_{\perp}} \lambda_{(V-A)_{\perp}^{\mathrm{adj}}}\right. \\
& +\frac{1}{N_{c}^{2}} \lambda_{(S+P)_{-}^{\mathrm{adj}}}^{2}-\frac{2}{N_{c}} \lambda_{(\sigma-\pi)} \lambda_{(S+P)_{-}^{\mathrm{adj}}}-\frac{4}{N_{c}} \lambda_{(S+P)_{-}} \lambda_{(S+P)_{-}^{\mathrm{adj}}}+\frac{1}{N_{c}} \lambda_{(V-A)_{\perp}^{\mathrm{adj}}}^{2}-2 \lambda_{(\sigma-\pi)} \lambda_{\mathrm{csc}} \\
& -4 \lambda_{(S+P)_{-}} \lambda_{\mathrm{csc}}+2 \lambda_{(S+P)_{-}^{\mathrm{adj}}} \lambda_{\mathrm{csc}}-\lambda_{(V-A)_{\|}} \lambda_{\mathrm{csc}}-3 \lambda_{(V-A)_{\perp}} \lambda_{\mathrm{csc}}+\frac{3}{2} \lambda_{(V-A)_{\perp}^{\mathrm{ajj}}} \lambda_{\mathrm{csc}} \\
& \left.+\frac{2}{N_{c}} \lambda_{(S+P)_{-}^{\mathrm{adj}}} \lambda_{\mathrm{csc}}+\frac{3}{2 N_{c}} \lambda_{(V-A)_{\perp}^{\mathrm{adj}}} \lambda_{\mathrm{csc}}+4 \lambda_{\mathrm{csc}}^{2}\right) l_{\perp \pm}^{(\mathrm{F})}\left(\tau, 0,-i \tilde{\mu}_{\tau}\right), \\
& \partial_{t} \lambda_{(S+P)_{-}^{\mathrm{adj}}}=2 \lambda_{(S+P)_{-}^{\mathrm{adj}}}+64 v_{4}\left(\lambda_{(\sigma-\pi)}^{2}+2 \lambda_{(S+P)_{-}^{\mathrm{adj}}} \lambda_{(V+A)_{\|}}-\frac{3}{2} \lambda_{(\sigma-\pi)} \lambda_{(V+A)_{\|}^{\mathrm{adj}}}+\lambda_{(S+P)_{-}} \lambda_{(V+A)_{\|}^{\mathrm{adj}}}\right. \\
& +\lambda_{(V+A)_{\|}^{\mathrm{adj}}}^{2}+2 \lambda_{(\sigma-\pi)} \lambda_{(V+A)_{\perp}}+4 \lambda_{(S+P)_{-}} \lambda_{(V+A)_{\perp}}+2 \lambda_{(S+P)_{-}^{\mathrm{adj}}} \lambda_{(V+A)_{\perp}}-3 \lambda_{(V-A)_{\perp}} \lambda_{(V-A)_{\perp}^{\mathrm{adj}}} \\
& -\frac{3}{2 N_{c}} \lambda_{(S+P)_{-}^{\mathrm{adj}}} \lambda_{(V+A)_{\|}^{\mathrm{adj}}}-\frac{2}{N_{c}} \lambda_{(S+P)_{-}^{\mathrm{adj}}} \lambda_{(V+A)_{\perp}}+\frac{3}{2 N_{c}} \lambda_{(V-A)_{\perp}^{\mathrm{adj}}}^{2}+\frac{N_{c}}{2} \lambda_{(S+P)_{-}^{\mathrm{adj}}} \lambda_{(V+A)_{\|}^{\mathrm{adj}}} \\
& -\frac{3 N_{c}}{4} \lambda_{(V-A)_{\perp}^{\mathrm{adj}}}^{2}+2 \lambda_{(V+A)_{\|}} \lambda_{\mathrm{csc}}-2 \lambda_{(V-A)_{\|}} \lambda_{\mathrm{csc}}-\frac{1}{2} \lambda_{(V+A)_{\|}^{\mathrm{adj}}} \lambda_{\mathrm{csc}}+\frac{3}{2} \lambda_{(V-A)_{\perp}^{\mathrm{adj}}} \lambda_{\mathrm{csc}} \\
& \left.-\frac{1}{N_{c}} \lambda_{(V+A)_{\|}^{\mathrm{adj}}} \lambda_{\mathrm{csc}}+\frac{N_{c}}{2} \lambda_{(V+A)_{\|}^{\mathrm{adj}}} \lambda_{\mathrm{csc}}-\frac{3 N_{c}}{2} \lambda_{(V-A)_{\perp}^{\mathrm{adj}}} \lambda_{\mathrm{csc}}+2 \lambda_{\mathrm{csc}}^{2}-N_{c} \lambda_{\mathrm{csc}}^{2}\right) l_{\|+}^{(\mathrm{F})}\left(\tau, 0,-i \tilde{\mu}_{\tau}\right)
\end{aligned}
$$




$$
\begin{aligned}
& +64 v_{4}\left(\lambda_{(\sigma-\pi)}^{2}+4 \lambda_{(\sigma-\pi)} \lambda_{(S+P)_{-}}+4 \lambda_{(S+P)_{-}}^{2}+4 \lambda_{(\sigma-\pi)} \lambda_{(S+P)_{-}^{\mathrm{adj}}}+8 \lambda_{(S+P)_{-}} \lambda_{(S+P)_{-}^{\mathrm{adj}}}\right. \\
& +\lambda_{(S+P)_{-}^{\mathrm{adj}}}^{2}-\lambda_{(S+P)_{-}^{\mathrm{adj}}} \lambda_{(V-A)_{\|}}+2 \lambda_{(\sigma-\pi)} \lambda_{(V-A)_{\perp}}+4 \lambda_{(S+P)_{-}} \lambda_{(V-A)_{\perp}}-\lambda_{(S+P)_{-}^{\mathrm{adj}}} \lambda_{(V-A)_{\perp}} \\
& -\frac{1}{2} \lambda_{(\sigma-\pi)} \lambda_{(V-A)_{\perp}^{\mathrm{adj}}}-\lambda_{(S+P)_{-}} \lambda_{(V-A)_{\perp}^{\mathrm{adj}}}+\lambda_{(S+P)_{-}^{\mathrm{adj}}} \lambda_{(V-A)_{\perp}^{\mathrm{adj}}}+3 \lambda_{(V-A) \perp} \lambda_{(V-A)_{\perp}^{\mathrm{adj}}}+\frac{1}{N_{c}^{2}} \lambda_{(S+P)_{-}^{\mathrm{adj}}}^{2} \\
& +\frac{1}{N_{c}^{2}} \lambda_{(S+P)_{-}^{a d j}} \lambda_{(V-A)_{\perp}^{\text {adj }}}-\frac{2}{N_{c}} \lambda_{(\sigma-\pi)} \lambda_{(S+P)_{-}^{\text {adj }}}-\frac{4}{N_{c}} \lambda_{(S+P)_{-}} \lambda_{(S+P)_{-}^{\text {adj }}}-\frac{4}{N_{c}} \lambda_{(S+P)_{-}^{\text {adj }}}^{2} \\
& \left.-\frac{2}{N_{c}} \lambda_{(S+P)_{-}^{\mathrm{adj}}} \lambda_{(V-A)_{\perp}}-\frac{1}{N_{c}} \lambda_{(\sigma-\pi)} \lambda_{(V-A)_{\perp}^{\mathrm{adj}}}-\frac{2}{N_{c}} \lambda_{(S+P)_{-}} \lambda_{(V-A)_{\perp}^{\mathrm{adj}}}+\frac{1}{N_{c}} \lambda_{(S+P)_{-}^{\mathrm{adj}}} \lambda_{(V-A)_{\perp}^{\mathrm{ajj}}}-\frac{3}{2 N_{c}} \lambda_{(V-A)_{\perp}^{2}}^{\mathrm{adj}}\right) l_{\| \pm}^{(\mathrm{F})}\left(\tau, 0,-i \tilde{\mu}_{\tau}\right)
\end{aligned}
$$

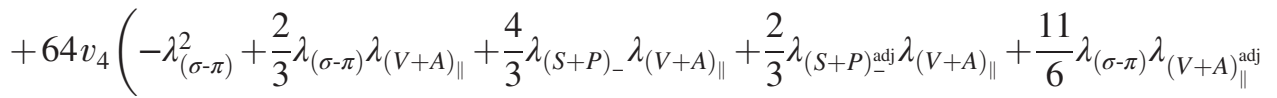

$$
\begin{aligned}
& -\frac{1}{3} \lambda_{(S+P)_{-}} \lambda_{(V+A)_{\|}^{\mathrm{adj}}}-\lambda_{(V+A)_{\|}^{\mathrm{adj}}}^{2}+\frac{4}{3} \lambda_{(\sigma-\pi)} \lambda_{(V+A)_{\perp}}+\frac{8}{3} \lambda_{(S+P)_{-}} \lambda_{(V+A)_{\perp}}+\frac{10}{3} \lambda_{(S+P)_{-}^{\mathrm{ajj}}} \lambda_{(V+A)_{\perp}} \\
& -\lambda_{(V-A)} \lambda_{(V-A)_{\perp}^{\mathrm{adj}}}+2 \lambda_{(V-A)_{\perp}} \lambda_{(V-A)_{\perp}^{\mathrm{adj}}}+\frac{1}{3 N_{c}^{2}} \lambda_{(S+P)_{-}^{\mathrm{adj}}} \lambda_{(V+A)_{\|}^{\mathrm{adj}}}-\frac{2}{3 N_{c}} \lambda_{(S+P)_{-}^{\mathrm{adj}}} \lambda_{(V+A)_{\|}} \\
& -\frac{1}{3 N_{c}} \lambda_{(\sigma-\pi)} \lambda_{(V+A)_{\|}^{\mathrm{ajj}}}-\frac{2}{3 N_{c}} \lambda_{(S+P)_{-}} \lambda_{(V+A)_{\|}^{\mathrm{adj}}}-\frac{1}{6 N_{c}} \lambda_{(S+P)_{-}^{\mathrm{adj}}} \lambda_{(V+A)_{\|}^{\mathrm{adj}}}-\frac{4}{3 N_{c}} \lambda_{(S+P)_{-}^{\mathrm{adj}}} \lambda_{(V+A)_{\perp}} \\
& -\frac{1}{N_{c}} \lambda_{(V-A)_{\perp}^{2}}^{\text {adj }}-\frac{N_{c}}{6} \lambda_{(S+P)_{-}^{\text {adj }}} \lambda_{(V+A)_{\|}^{\mathrm{ajj}}}+\frac{N_{c}}{2} \lambda_{(V-A)_{\perp}^{2}}^{\text {adj }}+\frac{1}{6} \lambda_{(V+A)_{\|}^{\mathrm{adj}}} \lambda_{\mathrm{csc}}+2 \lambda_{(V+A) \perp} \lambda_{\mathrm{csc}}
\end{aligned}
$$

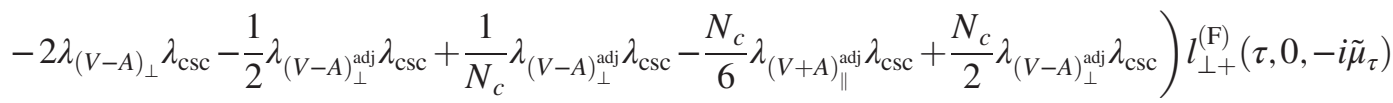

$$
\begin{aligned}
& +64 v_{4}\left(-\lambda_{(\sigma-\pi)}^{2}-4 \lambda_{(\sigma-\pi)} \lambda_{(S+P)_{-}}-4 \lambda_{(S+P)_{-}}^{2}-\lambda_{(S+P)_{-}^{\text {adj }}}^{2}+\frac{2}{3} \lambda_{(\sigma-\pi)} \lambda_{(V-A)_{\|}}+\frac{4}{3} \lambda_{(S+P)_{-}} \lambda_{(V-A)_{\|}}\right. \\
& -\frac{1}{3} \lambda_{(S+P)_{-}^{\mathrm{adj}}} \lambda_{(V-A)_{\|}}+\frac{4}{3} \lambda_{(\sigma-\pi)} \lambda_{(V-A)_{\perp}}+\frac{8}{3} \lambda_{(S+P)_{-}} \lambda_{(V-A)_{\perp}}-\frac{5}{3} \lambda_{(S+P)_{-}^{\mathrm{adj}}} \lambda_{(V-A)_{\perp}}
\end{aligned}
$$

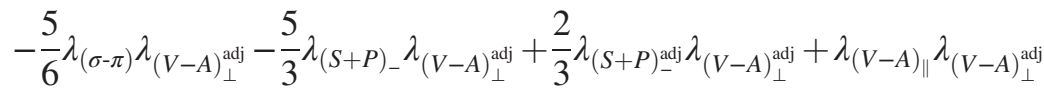

$$
\begin{aligned}
& +2 \lambda_{(V-A)_{\perp}} \lambda_{(V-A)_{\perp}^{\mathrm{adj}}}-\frac{1}{N_{c}^{2}} \lambda_{(S+P)_{-}^{\mathrm{ajj}}}^{2}+\frac{2}{3 N_{c}^{2}} \lambda_{(S+P)_{-}^{\mathrm{adj}}} \lambda_{(V-A)_{\perp}}{ }_{-}^{\mathrm{adj}}+\frac{2}{N_{c}} \lambda_{(\sigma-\pi)} \lambda_{(S+P)_{-}^{\mathrm{adj}}} \\
& +\frac{4}{N_{c}} \lambda_{(S+P)_{-}} \lambda_{(S+P)_{-}^{\mathrm{adj}}}-\frac{2}{3 N_{c}} \lambda_{(S+P)_{-}^{\mathrm{ajj}}} \lambda_{(V-A)_{\|}}-\frac{4}{3 N_{c}} \lambda_{(S+P)_{-}^{\mathrm{adj}}} \lambda_{(V-A)_{\perp}}-\frac{2}{3 N_{c}} \lambda_{(\sigma-\pi)} \lambda_{(V-A)_{\perp}^{\mathrm{ajj}}} \\
& \left.-\frac{4}{3 N_{c}} \lambda_{(S+P)_{-}} \lambda_{(V-A)_{\perp}^{\mathrm{adj}}}+\frac{5}{3 N_{c}} \lambda_{(S+P)_{-}^{\mathrm{adj}}} \lambda_{(V-A)_{\perp}^{\mathrm{adj}}}-\frac{1}{N_{c}} \lambda_{(V-A)_{\perp}^{2}}^{\mathrm{adj}}\right) l_{\perp \pm}^{(\mathrm{F})}\left(\tau, 0,-i \tilde{\mu}_{\tau}\right),
\end{aligned}
$$




$$
\begin{aligned}
& \partial_{t} \lambda_{(S+P)_{-}}=2 \lambda_{(S+P)_{-}}+64 v_{4}\left(-\frac{1}{2} \lambda_{(\sigma-\pi)}^{2}+\lambda_{(\sigma-\pi)} \lambda_{(S+P)_{-}}+2 \lambda_{(S+P)_{-}}^{2}+\frac{1}{2} \lambda_{(\sigma-\pi)} \lambda_{(V+A)_{\|}}+2 \lambda_{(S+P)_{-}} \lambda_{(V+A)_{\|}}\right. \\
& -\frac{1}{2} \lambda_{(\sigma-\pi)} \lambda_{(V-A)_{\|}}+\lambda_{(\sigma-\pi)} \lambda_{(V+A)_{\|}^{\mathrm{adj}}}+\frac{1}{4} \lambda_{(S+P)_{-}^{\mathrm{adj}}} \lambda_{(V+A)_{\|}^{\mathrm{adj}}}-\frac{1}{2} \lambda_{(V+A)_{\|}^{2}}^{\mathrm{adj}}-\frac{1}{2} \lambda_{(\sigma-\pi)} \lambda_{(V+A)_{\perp}} \\
& +2 \lambda_{(S+P)_{-}} \lambda_{(V+A)_{\perp}}+\lambda_{(S+P)_{-}^{\mathrm{adj}}} \lambda_{(V+A)_{\perp}}+\frac{1}{2} \lambda_{(V+A)_{\|}^{\mathrm{adj}}} \lambda_{(V+A)_{\perp}}-\frac{1}{2} \lambda_{(\sigma-\pi)} \lambda_{(V-A)_{\perp}} \\
& -\lambda_{(\sigma-\pi)} \lambda_{(V-A)_{\perp}^{\mathrm{adj}}}+\frac{3}{2} \lambda_{(V-A)_{\perp}} \lambda_{(V-A)_{\perp}^{\mathrm{adj}}}-\frac{3}{8} \lambda_{(V-A)_{\perp}^{\mathrm{adj}}}^{2}+\frac{1}{2 N_{c}^{2}} \lambda_{(S+P)_{-}^{\mathrm{adj}}}^{2}-\frac{1}{4 N_{c}^{2}} \lambda_{(S+P)_{-}^{\mathrm{adj}}} \lambda_{(V+A)_{\|}^{\mathrm{adj}}} \\
& -\frac{1}{N_{c}^{2}} \lambda_{(S+P)_{-}^{\mathrm{adj}}} \lambda_{(V+A)_{\perp}}+\frac{3}{4 N_{c}^{2}} \lambda_{(V-A)_{\perp}^{\mathrm{adj}}}^{2}+\frac{1}{2 N_{c}} \lambda_{(\sigma-\pi)}^{2}-\frac{1}{2 N_{c}} \lambda_{(\sigma-\pi)} \lambda_{(S+P)_{-}^{\mathrm{adj}}}-\frac{2}{N_{c}} \lambda_{(S+P)_{-}} \lambda_{(S+P)_{-}^{\mathrm{adj}}} \\
& -\frac{1}{2 N_{c}} \lambda_{(S+P)_{-}^{\mathrm{adj}}}^{2}-\frac{1}{N_{c}} \lambda_{(\sigma-\pi)} \lambda_{(V+A)_{\|}^{\mathrm{adj}}}-\frac{1}{2 N_{c}} \lambda_{(S+P)_{-}} \lambda_{(V+A)_{\|}^{\mathrm{adj}}}+\frac{1}{2 N_{c}} \lambda_{(V+A)_{\|}^{\mathrm{adj}}}^{2} \\
& +\frac{1}{N_{c}} \lambda_{(\sigma-\pi)} \lambda_{(V+A)_{\perp}}+\frac{2}{N_{c}} \lambda_{(S+P)_{-}} \lambda_{(V+A)_{\perp}}+\frac{1}{4 N_{c}} \lambda_{(\sigma-\pi)} \lambda_{(V-A)_{\perp}^{\mathrm{adj}}}-\frac{3}{2 N_{c}} \lambda_{(V-A)_{\perp}} \lambda_{(V-A)_{\perp}^{\mathrm{adj}}} \\
& -\frac{3}{4 N_{c}} \lambda_{(V-A)_{\perp}^{\mathrm{adj}}}^{2}+2 N_{c} \lambda_{(S+P)_{-}}^{2}+N_{c} \lambda_{(S+P)_{-}} \lambda_{(S+P)_{-}^{\mathrm{adj}}}+\frac{N_{c}}{2} \lambda_{(S+P)_{-}} \lambda_{(V+A)_{\|}^{\mathrm{adj}}}+\frac{3 N_{c}}{8} \lambda_{(V-A)_{\perp}^{\mathrm{adj}}}^{2} \\
& -\lambda_{(\sigma-\pi)} \lambda_{\mathrm{csc}}+\frac{1}{2} \lambda_{(S+P)_{-}^{\mathrm{adj}}} \lambda_{\mathrm{csc}}-\lambda_{(V+A)} \lambda_{\mathrm{csc}}+\lambda_{(V-A)_{\|}} \lambda_{\mathrm{csc}}+\frac{1}{2} \lambda_{(V+A)_{\|}^{\mathrm{ajj}}} \lambda_{\mathrm{csc}}-\frac{3}{2} \lambda_{(V-A)_{\perp}^{\mathrm{adj}}} \lambda_{\mathrm{csc}} \\
& -\frac{1}{2 N_{c}^{2}} \lambda_{(V+A)_{\|}^{\mathrm{adj}}} \lambda_{\mathrm{csc}}+\frac{1}{N_{c}} \lambda_{(V+A)} \lambda_{\mathrm{csc}}-\frac{1}{N_{c}} \lambda_{(V-A)_{\|}} \lambda_{\mathrm{csc}}+\frac{1}{4 N_{c}} \lambda_{(V+A)_{\|}^{\mathrm{adj}}} \lambda_{\mathrm{csc}}+\frac{3}{4 N_{c}} \lambda_{(V-A)_{\perp}^{\mathrm{adj}}} \lambda_{\mathrm{csc}} \\
& \left.-N_{c} \lambda_{(S+P)_{-}} \lambda_{\mathrm{csc}}-\frac{N_{c}}{2} \lambda_{(S+P)_{-}^{\mathrm{adj}}} \lambda_{\mathrm{csc}}-\frac{N_{c}}{4} \lambda_{(V+A)_{\|}^{\mathrm{adj}}} \lambda_{\mathrm{csc}}+\frac{3 N_{c}}{4} \lambda_{(V-A)_{\perp}^{\mathrm{adj}}} \lambda_{\mathrm{csc}}-\frac{3}{2} \lambda_{\mathrm{csc}}^{2}+\frac{1}{N_{c}} \lambda_{\mathrm{csc}}^{2}+\frac{N_{c}}{2} \lambda_{\mathrm{csc}}^{2}\right) l_{\|+}^{(\mathrm{F})}\left(\tau, 0,-i \tilde{\mu}_{\tau}\right) \\
& +64 v_{4}\left(-\frac{1}{2} \lambda_{(\sigma-\pi)}^{2}-2 \lambda_{(\sigma-\pi)} \lambda_{(S+P)_{-}}-2 \lambda_{(S+P)_{-}}^{2}-2 \lambda_{(\sigma-\pi)} \lambda_{(S+P)_{-}^{\mathrm{adj}}}-4 \lambda_{(S+P)_{-}} \lambda_{(S+P)_{-}^{\mathrm{adj}}}\right. \\
& -\frac{1}{2} \lambda_{(S+P)_{-}^{\text {adj }}}^{2}+\frac{1}{2} \lambda_{(\sigma-\pi)} \lambda_{(V+A)_{\|}}-\frac{1}{2} \lambda_{(\sigma-\pi)} \lambda_{(V-A)_{\|}}-\lambda_{(S+P)_{-}} \lambda_{(V-A)_{\|}}-\frac{1}{2} \lambda_{(\sigma-\pi)} \lambda_{(V+A)_{\perp}} \\
& -\frac{1}{2} \lambda_{(V+A)_{\|}^{\mathrm{adj}}} \lambda_{(V+A)_{\perp}}-\frac{1}{2} \lambda_{(\sigma-\pi)} \lambda_{(V-A)_{\perp}}-\lambda_{(S+P)_{-}} \lambda_{(V-A)_{\perp}}+\lambda_{(S+P)_{-}^{\mathrm{adj}}} \lambda_{(V-A)_{\perp}} \\
& +\frac{1}{2} \lambda_{(\sigma-\pi)} \lambda_{(V-A)_{\perp}^{\mathrm{adj}}}+\lambda_{(S+P)_{-}} \lambda_{(V-A)_{\perp}^{\mathrm{adj}}}-\frac{1}{4} \lambda_{(S+P)_{-}^{\mathrm{adj}}} \lambda_{(V-A)_{\perp}^{\mathrm{adj}}}-\frac{3}{2} \lambda_{(V-A)_{\perp}} \lambda_{(V-A)_{\perp}^{\mathrm{adj}}} \\
& +\frac{1}{2 N_{c}^{3}} \lambda_{(S+P)_{-}^{\mathrm{adj}}}^{2}+\frac{1}{2 N_{c}^{3}} \lambda_{(S+P)_{-}^{\mathrm{adj}}} \lambda_{(V-A)_{\perp}^{\mathrm{adj}}}-\frac{1}{N_{c}^{2}} \lambda_{(\sigma-\pi)} \lambda_{(S+P)_{-}^{\mathrm{adj}}}-\frac{2}{N_{c}^{2}} \lambda_{(S+P)_{-}} \lambda_{(S+P)_{-}^{\mathrm{adj}}} \\
& -\frac{5}{2 N_{c}^{2}} \lambda_{(S+P)_{-}^{\mathrm{adj}}}^{2}-\frac{1}{N_{c}^{2}} \lambda_{(S+P)_{-}^{\mathrm{adj}}} \lambda_{(V-A)_{\perp}}-\frac{1}{2 N_{c}^{2}} \lambda_{(\sigma-\pi)} \lambda_{(V-A)_{\perp}^{\mathrm{adj}}}-\frac{1}{N_{c}^{2}} \lambda_{(S+P)_{-}} \lambda_{(V-A)_{\perp}^{\mathrm{adj}}} \\
& +\frac{1}{4 N_{c}^{2}} \lambda_{(S+P)_{-}^{\mathrm{adj}}} \lambda_{(V-A)_{\perp}^{\mathrm{adj}}}-\frac{3}{4 N_{c}^{2}} \lambda_{(V-A)_{\perp}^{2}}^{\mathrm{adj}}+\frac{1}{2 N_{c}} \lambda_{(\sigma-\pi)}^{2}+\frac{2}{N_{c}} \lambda_{(\sigma-\pi)} \lambda_{(S+P)_{-}}+\frac{2}{N_{c}} \lambda_{(S+P)_{-}}^{2} \\
& +\frac{3}{N_{c}} \lambda_{(\sigma-\pi)} \lambda_{(S+P)_{-}^{\mathrm{adj}}}+\frac{6}{N_{c}} \lambda_{(S+P)_{-}} \lambda_{(S+P)_{-}^{\mathrm{adj}}}+\frac{5}{2 N_{c}} \lambda_{(S+P)_{-}^{\mathrm{adj}}}^{2}-\frac{1}{4 N_{c}} \lambda_{(\sigma-\pi)} \lambda_{(V+A)_{\|}^{\mathrm{adj}}} \\
& +\frac{1}{N_{c}} \lambda_{(\sigma-\pi)} \lambda_{(V-A)_{\perp}}+\frac{2}{N_{c}} \lambda_{(S+P)_{-}} \lambda_{(V-A)_{\perp}}-\frac{1}{2 N_{c}} \lambda_{(S+P)_{-}^{\mathrm{adj}}} \lambda_{(V-A)_{\perp}^{\mathrm{adj}}}+\frac{3}{2 N_{c}} \lambda_{(V-A)_{\perp}} \lambda_{(V-A)_{\perp}^{\mathrm{adj}}} \\
& \left.+\frac{3}{4 N_{c}} \lambda_{(V-A)_{\perp}^{\mathrm{adj}}}^{2}\right) l_{\| \pm}^{(\mathrm{F})}\left(\tau, 0,-i \tilde{\mu}_{\tau}\right) \\
& +64 v_{4}\left(\frac{1}{2} \lambda_{(\sigma-\pi)}^{2}+\lambda_{(\sigma-\pi)} \lambda_{(S+P)_{-}}+2 \lambda_{(S+P)_{-}}^{2}+\frac{1}{3} \lambda_{(\sigma-\pi)} \lambda_{(S+P)_{-}}^{\mathrm{adj}}+\frac{2}{3} \lambda_{(S+P)_{-}} \lambda_{(S+P)_{-}}{ }_{\mathrm{adj}}\right. \\
& -\frac{1}{6} \lambda_{(\sigma-\pi)} \lambda_{(V+A)_{\|}}+\frac{2}{3} \lambda_{(S+P)_{-}} \lambda_{(V+A)_{\|}}+\frac{1}{3} \lambda_{(S+P)_{-}^{a_{-}}} \lambda_{(V+A)_{\|}}-\frac{1}{6} \lambda_{(\sigma-\pi)} \lambda_{(V-A)_{\|}}-\frac{5}{6} \lambda_{(\sigma-\pi)} \lambda_{(V+A)_{\|}^{\mathrm{adj}}}
\end{aligned}
$$


$+\frac{1}{3} \lambda_{(S+P)_{-}} \lambda_{(V+A)_{\|}^{\mathrm{adj}}}-\frac{1}{12} \lambda_{(S+P)_{-}^{\mathrm{adj}}} \lambda_{(V+A)_{\|}^{\mathrm{adj}}}+\frac{1}{6} \lambda_{(V+A)_{\|}} \lambda_{(V+A)_{\|}^{\mathrm{adj}}}+\frac{1}{2} \lambda_{(V+A)_{\|}^{\mathrm{adj}}}^{2}+\frac{1}{6} \lambda_{(\sigma-\pi)} \lambda_{(V+A)_{\perp}}+\frac{10}{3} \lambda_{(S+P)_{-}} \lambda_{(V+A)_{\perp}}$

$+\frac{2}{3} \lambda_{(S+P)_{-}^{\mathrm{adj}}} \lambda_{(V+A)_{\perp}}+\frac{1}{3} \lambda_{(V+A)_{\|}^{\mathrm{adj}}} \lambda_{(V+A)_{\perp}}-\frac{1}{6} \lambda_{(\sigma-\pi)} \lambda_{(V-A) \perp}-\frac{1}{3} \lambda_{(\sigma-\pi)} \lambda_{(V-A)_{\perp}^{\mathrm{adj}}}+\frac{1}{2} \lambda_{(V-A)_{\|}} \lambda_{(V-A)_{\perp}^{\mathrm{adj}}}-\lambda_{(V-A) \perp} \lambda_{(V-A)_{\perp}^{\mathrm{adj}}}+\frac{1}{4} \lambda_{(V-A)_{\perp}^{2}}^{\mathrm{adj}}$

$+\frac{1}{6 N_{c}^{3}} \lambda_{(S+P)_{-}^{\mathrm{adj}}} \lambda_{(V+A)_{\|}^{\mathrm{adj}}}+\frac{1}{2 N_{c}^{2}} \lambda_{(S+P)_{-}^{\mathrm{adj}}}^{2}-\frac{1}{3 N_{c}^{2}} \lambda_{(S+P)_{-}^{\mathrm{adj}}} \lambda_{(V+A)_{\|}}-\frac{1}{6 N_{c}^{2}} \lambda_{(\sigma-\pi)} \lambda_{(V+A)_{\|}^{\mathrm{ajj}}}-\frac{1}{3 N_{c}^{2}} \lambda_{(S+P)_{-}} \lambda_{(V+A)_{\|}^{\mathrm{adj}}}$

$+\frac{1}{12 N_{c}^{2}} \lambda_{(S+P)_{-}^{\mathrm{adj}}} \lambda_{(V+A)_{\|}^{\mathrm{ajj}}}-\frac{2}{3 N_{c}^{2}} \lambda_{(S+P)_{-}^{\mathrm{adj}}} \lambda_{(V+A)_{\perp}}-\frac{1}{2 N_{c}^{2}} \lambda_{(V-A)_{\perp}^{\mathrm{adj}}}^{2}-\frac{1}{2 N_{c}} \lambda_{(\sigma-\pi)}^{2}-\frac{1}{2 N_{c}} \lambda_{(\sigma-\pi)} \lambda_{(S+P)_{-}}{ }_{-j}-\frac{2}{N_{c}} \lambda_{(S+P)_{-}} \lambda_{(S+P)_{-}}{ }_{\mathrm{adj}}$

$-\frac{5}{6 N_{c}} \lambda_{(S+P)_{-}^{\mathrm{adj}}}^{2}+\frac{1}{3 N_{c}} \lambda_{(\sigma-\pi)} \lambda_{(V+A)_{\|}}+\frac{2}{3 N_{c}} \lambda_{(S+P)_{-}} \lambda_{(V+A)_{\|}}+\frac{1}{N_{c}} \lambda_{(\sigma-\pi)} \lambda_{(V+A)_{\|}^{\mathrm{adj}}}-\frac{1}{2 N_{c}} \lambda_{(S+P)_{-}} \lambda_{(V+A)_{\|}^{\mathrm{adj}}}-\frac{1}{3 N_{c}} \lambda_{(S+P)_{-}^{\mathrm{adj}}} \lambda_{(V+A)_{\|}^{\mathrm{adj}}}$

$-\frac{7}{12 N_{c}} \lambda_{(V+A)_{\|}^{\mathrm{adj}}}^{2}+\frac{2}{3 N_{c}} \lambda_{(\sigma-\pi)} \lambda_{(V+A)_{\perp}}+\frac{4}{3 N_{c}} \lambda_{(S+P)_{-}} \lambda_{(V+A)_{\perp}}+\frac{1}{12 N_{c}} \lambda_{(\sigma-\pi)} \lambda_{(V-A)_{\perp}^{\mathrm{adj}}}-\frac{1}{2 N_{c}} \lambda_{(V-A)_{\|}} \lambda_{(V-A)_{\perp}^{\mathrm{adj}}}$

$+\frac{1}{N_{c}} \lambda_{(V-A)_{\perp}} \lambda_{(V-A)_{\perp}^{\mathrm{adj}}}+\frac{1}{2 N_{c}} \lambda_{(V-A)_{\perp}^{2}}^{2}+2 N_{c} \lambda_{(S+P)_{-}}^{2}+N_{c} \lambda_{(S+P)_{-}} \lambda_{(S+P)_{-}^{\mathrm{adj}}}+\frac{N_{c}}{6} \lambda_{(S+P)_{-}^{\mathrm{adj}}}+\frac{N_{c}}{2} \lambda_{(S+P)_{-}} \lambda_{(V+A)_{\|}^{\mathrm{adj}}}$

$+\frac{N_{c}}{6} \lambda_{(S+P)_{-}^{\mathrm{adj}}} \lambda_{(V+A)_{\|}^{\mathrm{adj}}}+\frac{N_{c}}{24} \lambda_{(V+A)_{\|}^{\mathrm{adj}}}-\frac{N_{c}}{4} \lambda_{(V-A)_{\perp}^{\mathrm{adj}}}^{2}-\frac{1}{3} \lambda_{(\sigma-\pi)} \lambda_{\mathrm{csc}}+\frac{2}{3} \lambda_{(S+P)_{-}} \lambda_{\mathrm{csc}}+\frac{1}{6} \lambda_{\left.(S+P)_{-}\right)_{j} \lambda_{\mathrm{csc}}}-\lambda_{(V+A))_{\perp}} \lambda_{\mathrm{csc}}$

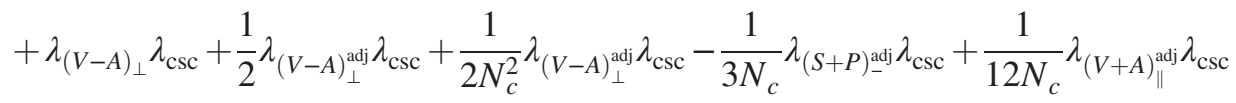

$+\frac{1}{N_{c}} \lambda_{(V+A)_{\perp}} \lambda_{\mathrm{csc}}-\frac{1}{N_{c}} \lambda_{(V-A)_{\perp}} \lambda_{\mathrm{csc}}-\frac{3}{4 N_{c}} \lambda_{(V-A)_{\perp}^{\mathrm{adj}}} \lambda_{\mathrm{csc}}-N_{c} \lambda_{(S+P)_{-}} \lambda_{\mathrm{csc}}-\frac{N_{c}}{6} \lambda_{\left.(S+P)_{-}\right)_{j}} \lambda_{\mathrm{csc}}$

$\left.-\frac{N_{c}}{12} \lambda_{(V+A)_{\|}^{\mathrm{adj}}} \lambda_{\mathrm{csc}}-\frac{N_{c}}{4} \lambda_{(V-A)_{\perp}^{\mathrm{adj}}} \lambda_{\mathrm{csc}}-\frac{1}{3} \lambda_{\mathrm{csc}}^{2}+\frac{N_{c}}{6} \lambda_{\mathrm{csc}}^{2}\right) l_{\perp+}^{(\mathrm{F})}\left(\tau, 0,-i \tilde{\mu}_{\tau}\right)$

$+64 v_{4}\left(\frac{1}{2} \lambda_{(\sigma-\pi)}^{2}+2 \lambda_{(\sigma-\pi)} \lambda_{(S+P)_{-}}+2 \lambda_{(S+P)_{-}}^{2}+\frac{1}{2} \lambda_{(S+P)_{-}^{\mathrm{ajj}}}^{2}-\frac{1}{6} \lambda_{(\sigma-\pi)} \lambda_{(V+A)_{\|}}-\frac{1}{6} \lambda_{(\sigma-\pi)} \lambda_{(V-A)_{\|}}\right.$

$-\frac{1}{3} \lambda_{(S+P)_{-}} \lambda_{(V-A)_{\|}}+\frac{1}{3} \lambda_{(S+P)_{-}} \lambda_{(V-A)_{\|}}-\frac{1}{6} \lambda_{(V+A)_{\|}} \lambda_{(V+A)_{\|}^{\mathrm{adj}}}+\frac{5}{6} \lambda_{(\sigma-\pi)} \lambda_{(V+A)_{\perp}}$

$+\frac{1}{3} \lambda_{(V+A)_{\|}^{\mathrm{ajj}}} \lambda_{(V+A)_{\perp}}-\frac{5}{6} \lambda_{(\sigma-\pi)} \lambda_{(V-A)_{\perp}}-\frac{5}{3} \lambda_{(S+P)_{-}} \lambda_{(V-A)_{\perp}}+\frac{2}{3} \lambda_{(S+P)_{-}^{\mathrm{ajj}}} \lambda_{(V-A)_{\perp}}$

$+\frac{1}{3} \lambda_{(\sigma-\pi)} \lambda_{(V-A)_{\perp}^{\mathrm{adj}}}+\frac{2}{3} \lambda_{(S+P)_{-}} \lambda_{(V-A)_{\perp}^{\mathrm{adj}}}-\frac{5}{12} \lambda_{(S+P)_{-}^{\mathrm{adj}}} \lambda_{(V-A)_{\perp}^{\mathrm{adj}}}-\frac{1}{2} \lambda_{(V-A)} \lambda_{(V-A)_{\perp}^{\mathrm{adj}}}$

$-\lambda_{(V-A)_{\perp}} \lambda_{(V-A)_{\perp}^{\mathrm{adj}}}-\frac{1}{2 N_{c}^{3}} \lambda_{(S+P)_{-}^{2}}^{2}+\frac{1}{3 N_{c}^{3}} \lambda_{(S+P)_{-}^{\mathrm{adj}}} \lambda_{(V-A)_{\perp}}^{\mathrm{adj}}+\frac{1}{N_{c}^{2}} \lambda_{(\sigma-\pi)} \lambda_{(S+P)_{-}^{\mathrm{adj}}}$

$+\frac{2}{N_{c}^{2}} \lambda_{(S+P)_{-}} \lambda_{(S+P)_{-}^{\mathrm{ajj}}}+\frac{1}{2 N_{c}^{2}} \lambda_{(S+P)_{-}^{2}}^{\text {adj }}-\frac{1}{3 N_{c}^{2}} \lambda_{(S+P)_{-}^{\mathrm{adj}}} \lambda_{(V-A)_{\|}}-\frac{2}{3 N_{c}^{2}} \lambda_{(S+P)_{-}^{\mathrm{adj}}} \lambda_{(V-A)_{\perp}}$

$-\frac{1}{3 N_{c}^{2}} \lambda_{(\sigma-\pi)} \lambda_{(V-A)_{\perp}^{\mathrm{adj}}}-\frac{2}{3 N_{c}^{2}} \lambda_{(S+P)_{-}} \lambda_{(V-A)_{\perp}^{\mathrm{adj}}}+\frac{5}{12 N_{c}^{2}} \lambda_{(S+P)_{-}^{\mathrm{adj}}} \lambda_{(V-A)_{\perp}} \mathrm{ajj}-\frac{1}{2 N_{c}^{2}} \lambda_{(V-A)_{\perp}^{2}}^{\mathrm{adj}}$

$-\frac{1}{2 N_{c}} \lambda_{(\sigma-\pi)}^{2}-\frac{2}{N_{c}} \lambda_{(\sigma-\pi)} \lambda_{(S+P)_{-}}-\frac{2}{N_{c}} \lambda_{(S+P)_{-}}^{2}-\frac{1}{N_{c}} \lambda_{(\sigma-\pi)} \lambda_{(S+P)_{-}} \mathrm{adj}_{-}-\frac{2}{N_{c}} \lambda_{(S+P)_{-}} \lambda_{(S+P)_{-}}$

$-\frac{1}{2 N_{c}} \lambda_{(S+P)_{-}^{\text {adj }}}^{2}+\frac{1}{3 N_{c}} \lambda_{(\sigma-\pi)} \lambda_{(V-A)_{\|}}+\frac{2}{3 N_{c}} \lambda_{(S+P)_{-}} \lambda_{(V-A)_{\|}}+\frac{1}{12 N_{c}} \lambda_{(\sigma-\pi)} \lambda_{(V+A)_{\|}^{\text {adj }}}$

$+\frac{1}{12 N_{c}} \lambda_{(V+A)_{\|}^{\mathrm{adj}}}^{2}+\frac{2}{3 N_{c}} \lambda_{(\sigma-\pi)} \lambda_{(V-A)_{\perp}}+\frac{4}{3 N_{c}} \lambda_{(S+P)_{-}} \lambda_{(V-A)_{\perp}}-\frac{1}{3 N_{c}} \lambda_{(S+P)_{-}^{\mathrm{adj}}} \lambda_{(V-A)_{\perp}^{\mathrm{adj}}}$

$+\frac{1}{2 N_{c}} \lambda_{(V-A)_{\|}} \lambda_{(V-A)_{\perp}^{\mathrm{ajj}}}+\frac{1}{N_{c}} \lambda_{(V-A)_{\perp}} \lambda_{(V-A)_{\perp}^{\mathrm{adj}}}+\frac{1}{2 N_{c}} \lambda_{(V-A)_{\perp}^{\mathrm{adj}}}^{2} l_{\perp \pm}^{(\mathrm{F})}\left(\tau, 0,-i \tilde{\mu}_{\tau}\right)$, 


$$
\begin{aligned}
& \partial_{t} \lambda_{(V+A)_{\|}}=2 \lambda_{(V+A)_{\|}}+64 v_{4}\left(\frac{1}{2} \lambda_{(\sigma-\pi)}^{2}+2 \lambda_{(\sigma-\pi)} \lambda_{(S+P)_{-}}+2 \lambda_{(S+P)_{-}}^{2}+\frac{1}{2} \lambda_{(\sigma-\pi)} \lambda_{(S+P)_{-}^{\mathrm{adj}}}+\lambda_{(S+P)_{-}} \lambda_{(S+P)_{-}^{\mathrm{adj}}}\right. \\
& +\lambda_{(S+P)_{-}^{\mathrm{adj}}}^{2}+\frac{1}{2} \lambda_{(V+A)_{\|}}^{2}+2 \lambda_{(\sigma-\pi)} \lambda_{(V-A)_{\|}}+\lambda_{(V+A)_{\|}} \lambda_{(V-A)_{\|}}+\frac{1}{8} \lambda_{(V+A)_{\|}^{\mathrm{adj}}}^{2}+\frac{3}{2} \lambda_{(V+A)_{\perp}}^{2} \\
& -3 \lambda_{(V+A)_{\|}} \lambda_{(V-A)_{\perp}}+\frac{3}{4} \lambda_{(\sigma-\pi)} \lambda_{(V-A)_{\perp}^{\mathrm{adj}}}-\frac{1}{2 N_{c}^{2}} \lambda_{(S+P)_{-}^{\mathrm{adj}}}^{2}-\frac{1}{8 N_{c}^{2}} \lambda_{(V+A)_{\|}^{\mathrm{adj}}}-\frac{1}{N_{c}^{2}} \lambda_{(\sigma-\pi)} \lambda_{(V-A)_{\perp}^{\mathrm{adj}}} \\
& -\frac{1}{2 N_{c}} \lambda_{(S+P)_{-}^{\mathrm{adj}}}^{2}-\frac{1}{2 N_{c}} \lambda_{(V+A)_{\|}^{\mathrm{adj}}} \lambda_{(V+A)_{\perp}}+\frac{2}{N_{c}} \lambda_{(\sigma-\pi)} \lambda_{(V-A)_{\perp}}+\frac{1}{N_{c}} \lambda_{(\sigma-\pi)} \lambda_{(V-A)_{\perp}^{\mathrm{adj}}} \\
& +\frac{3}{2 N_{c}} \lambda_{(V+A)_{\|}} \lambda_{(V-A)_{\perp}^{\mathrm{adj}}}-4 N_{c} \lambda_{(V+A)_{\|}} \lambda_{(V-A)_{\|}}-\frac{3 N_{c}}{2} \lambda_{(V+A)_{\|}} \lambda_{(V-A)_{\perp}^{\mathrm{adj}}}-\lambda_{(\sigma-\pi)} \lambda_{\mathrm{csc}}-\lambda_{(S+P)_{-}} \lambda_{\mathrm{csc}} \\
& +\frac{3}{2} \lambda_{(S+P)_{-}}^{\mathrm{adj}} \lambda_{\mathrm{csc}}-\lambda_{(V+A)_{\|}} \lambda_{\mathrm{csc}}-\frac{1}{N_{c}^{2}} \lambda_{(S+P)_{-}^{\mathrm{adj}}} \lambda_{\mathrm{csc}}+\frac{2}{N_{c}} \lambda_{(\sigma-\pi)} \lambda_{\mathrm{csc}}+\frac{2}{N_{c}} \lambda_{(S+P)_{-}} \lambda_{\mathrm{csc}} \\
& \left.-\frac{1}{2 N_{c}} \lambda_{(S+P)_{-}^{\mathrm{adj}}} \lambda_{\mathrm{csc}}+N_{c} \lambda_{(V+A)_{\|}} \lambda_{\mathrm{csc}}+\lambda_{\mathrm{csc}}^{2}-\frac{1}{N_{c}} \lambda_{\mathrm{csc}}^{2}\right) l_{\|+}^{(\mathrm{F})}\left(\tau, 0,-i \tilde{\mu}_{\tau}\right) \\
& +64 v_{4}\left(-\frac{1}{2} \lambda_{(\sigma-\pi)}^{2}-\frac{1}{2} \lambda_{(V+A)_{\|}}^{2}+\frac{1}{4} \lambda_{(\sigma-\pi)} \lambda_{(V+A)_{\|}^{\mathrm{adj}}}-\frac{1}{8} \lambda_{(V+A)_{\|}^{2}}^{\mathrm{adj}}-\frac{3}{2} \lambda_{(V+A)_{\perp}}^{2}\right. \\
& \left.+\frac{1}{8 N_{c}^{2}} \lambda_{(V+A)_{\|}^{\mathrm{adj}}}^{2}+\frac{2}{N_{c}} \lambda_{(\sigma-\pi)} \lambda_{(V+A)_{\perp}}+\frac{1}{2 N_{c}} \lambda_{(V+A)_{\|}^{\mathrm{adj}}} \lambda_{(V+A)_{\perp}}\right) l_{\| \pm}^{(\mathrm{F})}\left(\tau, 0,-i \tilde{\mu}_{\tau}\right) \\
& +64 v_{4}\left(\frac{1}{2} \lambda_{(\sigma-\pi)} \lambda_{(S+P)_{-}^{\mathrm{adj}}}+\lambda_{(S+P)_{-}} \lambda_{(S+P)_{-}^{\mathrm{adj}}}+\frac{1}{3} \lambda_{(S+P)_{-}^{\mathrm{adj}}}^{2}-2 \lambda_{(\sigma-\pi)} \lambda_{(V-A)_{\|}}-\lambda_{(V+A)_{\|}} \lambda_{(V-A)_{\|}}\right. \\
& -\frac{1}{24} \lambda_{(V+A)_{\|}^{\mathrm{adj}}}^{2}+\lambda_{(V+A)_{\|}} \lambda_{(V+A)_{\perp}}+\lambda_{(V+A)_{\perp}}^{2}+3 \lambda_{(V+A)} \lambda_{(V-A)_{\perp}}-\frac{3}{4} \lambda_{(\sigma-\pi)} \lambda_{(V-A)_{\perp}^{\mathrm{adj}}} \\
& +\frac{1}{3 N_{c}^{2}} \lambda_{(S+P)_{-}^{\mathrm{adj}}}^{2}+\frac{1}{12 N_{c}^{2}} \lambda_{(V+A)_{\|}^{\mathrm{adj}}}^{2}+\frac{2}{3 N_{c}^{2}} \lambda_{(\sigma-\pi)} \lambda_{(V-A)_{\perp}^{\mathrm{adj}}}-\frac{1}{3 N_{c}} \lambda_{(\sigma-\pi)} \lambda_{(S+P)_{-}^{\mathrm{adj}}} \\
& -\frac{2}{3 N_{c}} \lambda_{(S+P)_{-}} \lambda_{(S+P)_{-}^{\mathrm{adj}}}-\frac{1}{2 N_{c}} \lambda_{(S+P)_{-}^{\mathrm{adj}}}^{2}+\frac{2}{3 N_{c}} \lambda_{(\sigma-\pi)} \lambda_{(V-A)_{\|}}-\frac{1}{6 N_{c}} \lambda_{(V+A)_{\|}} \lambda_{(V+A)_{\|}^{\mathrm{adj}}} \\
& -\frac{1}{3 N_{c}} \lambda_{(V+A)_{\|}^{\mathrm{adj}}} \lambda_{(V+A)_{\perp}}-\frac{4}{3 N_{c}} \lambda_{(\sigma-\pi)} \lambda_{(V-A)_{\perp}}+\frac{1}{3 N_{c}} \lambda_{(\sigma-\pi)} \lambda_{(V-A)_{\perp}^{\mathrm{adj}}}-\frac{3}{2 N_{c}} \lambda_{(V+A)_{\|}} \lambda_{(V-A)_{\perp}^{\mathrm{adj}}} \\
& +4 N_{c} \lambda_{(V+A)_{\|}} \lambda_{(V-A)_{\|}}+\frac{3 N_{c}}{2} \lambda_{(V+A)_{\|}} \lambda_{(V-A)_{\perp}^{\mathrm{adj}}}+\lambda_{(\sigma-\pi)} \lambda_{\mathrm{csc}}+\lambda_{(S+P)_{-}} \lambda_{\mathrm{csc}}+\frac{1}{6} \lambda_{(S+P)_{-}^{\mathrm{ajj}}} \lambda_{\mathrm{csc}} \\
& +\lambda_{(V+A)_{\|}} \lambda_{\mathrm{csc}}+\frac{1}{3 N_{c}^{2}} \lambda_{(S+P)_{-}^{\mathrm{adj}}} \lambda_{\mathrm{csc}}-\frac{2}{3 N_{c}} \lambda_{(\sigma-\pi)} \lambda_{\mathrm{csc}}-\frac{2}{3 N_{c}} \lambda_{(S+P)_{-}} \lambda_{\mathrm{csc}}-\frac{1}{6 N_{c}} \lambda_{(S+P)_{-}^{\mathrm{djj}}} \lambda_{\mathrm{csc}} \\
& \left.-N_{c} \lambda_{(V+A) \|} \lambda_{\mathrm{csc}}-\frac{1}{6} \lambda_{\mathrm{csc}}^{2}+\frac{1}{3 N_{c}} \lambda_{\mathrm{csc}}^{2}\right) l_{\perp+}^{(\mathrm{F})}\left(\tau, 0,-i \tilde{\mu}_{\tau}\right) \\
& +64 v_{4}\left(\frac{1}{4} \lambda_{(\sigma-\pi)} \lambda_{(V+A)_{\|}^{\mathrm{adj}}}-\lambda_{(V+A)_{\|}} \lambda_{(V+A)_{\perp}}+\lambda_{(V+A)_{\perp}}^{2}-\frac{1}{3 N_{c}^{2}} \lambda_{(\sigma-\pi)} \lambda_{(V+A)_{\|}^{\mathrm{adj}}}-\frac{1}{12 N_{c}^{2}} \lambda_{(V+A)_{\|}^{2}}^{\mathrm{adj}}\right. \\
& +\frac{2}{3 N_{c}} \lambda_{(\sigma-\pi)} \lambda_{(V+A)_{\|}}+\frac{1}{6 N_{c}} \lambda_{(V+A)_{\|}} \lambda_{(V+A)_{\|}^{\text {adj }}}-\frac{4}{3 N_{c}} \lambda_{(\sigma-\pi)} \lambda_{(V+A)_{\perp}} \\
& \left.-\frac{1}{3 N_{c}} \lambda_{(V+A)_{\|}^{\mathrm{adj}}} \lambda_{(V+A)_{\perp}}\right) l_{\perp \pm}^{(\mathrm{F})}\left(\tau, 0,-i \tilde{\mu}_{\tau}\right)
\end{aligned}
$$




$$
\begin{aligned}
& \partial_{t} \lambda_{(V+A)_{\perp}}=2 \lambda_{(V+A)_{\perp}}+64 v_{4}\left(-\frac{1}{2 N_{c}} \lambda_{(S+P)_{-}}^{2}+\frac{1}{2} \lambda_{(S+P)_{-}^{\mathrm{adj}}}^{2}+\frac{1}{2} \lambda_{\mathrm{csc}} \lambda_{(S+P)_{-}^{\mathrm{adj}}}+\frac{1}{2} \lambda_{(\sigma-\pi)} \lambda_{(S+P)_{-}^{\mathrm{adj}}}\right. \\
& +\lambda_{(S+P)_{-}} \lambda_{(S+P)_{-}^{\mathrm{ajj}}}-\frac{1}{2 N_{c}} \lambda_{\mathrm{csc}} \lambda_{(S+P)_{-}}{ }_{-}+\lambda_{(V+A)_{\perp}}^{2}+\lambda_{\mathrm{csc}} \lambda_{(\sigma-\pi)}+\lambda_{\mathrm{csc}} \lambda_{(S+P)_{-}} \\
& -2 \lambda_{(\sigma-\pi)} \lambda_{(V-A)_{\perp}}-\frac{1}{4} \lambda_{(\sigma-\pi)} \lambda_{(V-A)_{\perp}^{\mathrm{adj}}}+\lambda_{\mathrm{csc}} \lambda_{(V+A)_{\perp}}+\lambda_{(V-A)_{\|}} \lambda_{(V+A)_{\perp}}+\lambda_{(V-A)_{\perp}} \lambda_{(V+A)_{\perp}} \\
& +\lambda_{(V+A) \|} \lambda_{(V+A)_{\perp}}-N_{c} \lambda_{\mathrm{csc}} \lambda_{(V+A)_{\perp}}+4 N_{c} \lambda_{(V-A) \perp} \lambda_{(V+A)_{\perp}}+\frac{N_{c}}{2} \lambda_{(V-A)_{\perp}} \lambda_{(V+A)_{\perp}} \\
& \left.+\frac{1}{N_{c}} \lambda_{(\sigma-\pi)} \lambda_{(V-A) \perp}{ }_{\perp}^{\text {adj }}-\frac{1}{2 N_{c}} \lambda_{(V-A)_{\perp}^{\mathrm{adj}}} \lambda_{(V+A)_{\perp}}-\frac{1}{2 N_{c}} \lambda_{(V+A)_{\|}^{\mathrm{adj}}} \lambda_{(V+A)_{\perp}}\right) l_{\|+}^{(\mathrm{F})}\left(\tau, 0,-\mathrm{i} \tilde{\mu}_{\tau}\right) \\
& +64 v_{4}\left(\lambda_{(V+A)_{\perp}}^{2}-\lambda_{(V+A)_{\|}} \lambda_{(V+A)_{\perp}}+\frac{1}{2 N_{c}} \lambda_{(V+A)_{\|}^{\mathrm{adj}}} \lambda_{(V+A)_{\perp}}+\frac{1}{4} \lambda_{(\sigma-\pi)} \lambda_{(V+A)_{\|}^{\mathrm{adj}}}\right) l_{\| \pm}^{(\mathrm{F})}\left(\tau, 0,-\mathrm{i} \tilde{\mu}_{\tau}\right) \\
& +64 v_{4}\left(\frac{1}{6} \lambda_{\mathrm{csc}}^{2}+\frac{1}{3} \lambda_{(\sigma-\pi)} \lambda_{\mathrm{csc}}+\frac{1}{3} \lambda_{(S+P)_{-}} \lambda_{\mathrm{csc}}+\frac{1}{2} \lambda_{(S+P)_{-}^{\mathrm{adj}}} \lambda_{\mathrm{csc}}+\frac{1}{3} \lambda_{(V+A)_{\perp}} \lambda_{\mathrm{csc}}\right. \\
& -\frac{N_{c}}{3} \lambda_{(V+A)_{\perp}} \lambda_{\mathrm{csc}}-\frac{1}{6 N_{c}} \lambda_{(S+P)_{-}^{\mathrm{adj}}} \lambda_{\mathrm{csc}}+\frac{1}{6} \lambda_{(\sigma-\pi)}^{2}+\frac{2}{3} \lambda_{(S+P)_{-}}^{2}+\frac{1}{2} \lambda_{(S+P)_{-}^{\mathrm{adj}}}^{2}+\frac{1}{6} \lambda_{(V+A)_{\|}}^{2} \\
& +\frac{7}{6} \lambda_{(V+A)_{\perp}}^{2}+\frac{2}{3} \lambda_{(\sigma-\pi)} \lambda_{(S+P)_{-}}+\frac{1}{2} \lambda_{(\sigma-\pi)} \lambda_{(S+P)_{-}^{\mathrm{adj}}}+\lambda_{(S+P)_{-}} \lambda_{(S+P)_{-}^{\mathrm{adj}}}-\frac{2}{3} \lambda_{(\sigma-\pi)} \lambda_{(V-A)_{\perp}} \\
& -\frac{1}{12} \lambda_{(\sigma-\pi)} \lambda_{(V-A)_{\perp}^{\mathrm{adj}}}+\frac{1}{3} \lambda_{(V-A) \|} \lambda_{(V+A)_{\perp}}+\frac{1}{3} \lambda_{(V-A)_{\perp}} \lambda_{(V+A)_{\perp}}+\frac{2}{3} \lambda_{(V+A)_{\|}} \lambda_{(V+A)_{\perp}} \\
& +\frac{4}{3} N_{c} \lambda_{(V-A)_{\perp}} \lambda_{(V+A)_{\perp}}+\frac{N_{c}}{6} \lambda_{(V-A)_{\perp}^{\mathrm{ajj}}} \lambda_{(V+A)_{\perp}}-\frac{1}{2 N_{c}} \lambda_{(S+P)_{-}^{\mathrm{adj}}}^{2}-\frac{1}{3 N_{c}} \lambda_{(\sigma-\pi)} \lambda_{(S+P)_{-}^{\mathrm{adj}}} \\
& -\frac{2}{3 N_{c}} \lambda_{(S+P)_{-}} \lambda_{(S+P)_{-}^{\mathrm{adj}}}+\frac{1}{3 N_{c}} \lambda_{(\sigma-\pi)} \lambda_{(V-A)_{\perp}^{\mathrm{adj}}}-\frac{1}{6 N_{c}} \lambda_{(V+A)_{\|}} \lambda_{(V+A)_{\|}^{\mathrm{adj}}}-\frac{1}{6 N_{c}} \lambda_{(V-A)_{\perp}^{\mathrm{ajj}}} \lambda_{(V+A)_{\perp}} \\
& \left.-\frac{1}{3 N_{c}} \lambda_{(V+A)_{\|}^{\mathrm{ajj}}} \lambda_{(V+A)_{\perp}}+\frac{1}{6 N_{c}^{2}} \lambda_{(S+P)_{-}^{\mathrm{adj}}}^{2}+\frac{1}{24 N_{c}^{2}} \lambda_{(V+A)_{\|}^{2}}^{\text {adj }}\right) l_{\perp+}^{(\mathrm{F})}\left(\tau, 0,-\mathrm{i} \tilde{\mu}_{\tau}\right) \\
& +64 v_{4}\left(-\frac{1}{6} \lambda_{(\sigma-\pi)}^{2}-\frac{1}{12} \lambda_{(V+A)_{\|}^{\text {adj }}} \lambda_{(\sigma-\pi)}-\frac{1}{6} \lambda_{(V+A)_{\|}}^{2}-\frac{1}{24} \lambda_{(V+A)_{\|}^{2}}^{\text {adj }}-\frac{7}{6} \lambda_{(V+A)_{\perp}}^{2}+\frac{2}{3} \lambda_{(V+A)_{\|}} \lambda_{(V+A)_{\perp}}\right. \\
& \left.+\frac{1}{6 N_{c}} \lambda_{(V+A)_{\|}} \lambda_{(V+A)_{\|}^{\mathrm{adj}}}-\frac{1}{3 N_{c}} \lambda_{(V+A)_{\|}^{\mathrm{adj}}} \lambda_{(V+A)_{\perp}}-\frac{1}{24 N_{c}^{2}} \lambda_{(V+A)_{\|}^{2}}^{\mathrm{adj}}\right) l_{\perp \pm}^{\mathrm{F})}\left(\tau, 0,-\mathrm{i} \tilde{\mu}_{\tau}\right), \\
& \partial_{t} \lambda_{(V-A)_{\|}}=2 \lambda_{(V-A)_{\|}}+64 v_{4}\left(\frac{1}{2} N_{c} \lambda_{\mathrm{csc}}^{2}-\frac{1}{2} \lambda_{\mathrm{csc}}^{2}-\lambda_{(V-A)} \lambda_{\mathrm{csc}}-\frac{3}{2} \lambda_{(V-A)_{\perp}^{\mathrm{adj}}} \lambda_{\mathrm{csc}}+N_{c} \lambda_{(V-A)} \lambda_{\mathrm{csc}}+\frac{3}{2} N_{c} \lambda_{(V-A)_{\perp}^{\mathrm{adj}}} \lambda_{\mathrm{csc}}-\frac{1}{2} \lambda_{(\sigma-\pi)}^{2}\right. \\
& +\frac{3}{2} \lambda_{(V-A)_{\|}}^{2}+\frac{3}{2} \lambda_{(V-A)_{\perp}}^{2}-\frac{1}{2} \lambda_{(V+A)_{\|}^{\mathrm{adj}}}-3 \lambda_{(V-A)_{\|}} \lambda_{(V-A)_{\perp}}+\frac{3}{2} \lambda_{(V-A)_{\perp}} \lambda_{(V-A)_{\perp}^{\mathrm{adj}}}+2 \lambda_{(\sigma-\pi)} \lambda_{(V+A)_{\|}}+\lambda_{(\sigma-\pi)} \lambda_{(V+A)_{\|}^{\mathrm{adj}}} \\
& \left.-2 N_{c} \lambda_{(V-A) \|}^{2}+\frac{3}{8} N_{c} \lambda_{(V-A)_{\perp}^{2}}^{\mathrm{adj}}-2 N_{c} \lambda_{(V+A) \|}^{2}-\frac{3}{2} N_{c} \lambda_{(V-A) \|} \lambda_{(V-A)}\right)_{\perp}^{\mathrm{ajj}}-\frac{3}{4 N_{c}} \lambda_{(V-A)_{\perp}^{\mathrm{adj}}}^{2}+\frac{1}{2 N_{c}} \lambda_{(V+A)_{\|}^{\mathrm{adj}}}^{2} \\
& \left.+\frac{3}{2 N_{c}} \lambda_{(V-A)} \lambda_{(V-A)_{\perp}^{\mathrm{adj}}}-\frac{3}{2 N_{c}} \lambda_{(V-A)_{\perp}} \lambda_{(V-A)_{\perp}^{\mathrm{adj}}}-\frac{1}{N_{c}} \lambda_{(\sigma-\pi)} \lambda_{(V+A)_{\|}^{\mathrm{adj}}}+\frac{3}{8 N_{c}^{2}} \lambda_{(V-A)_{\perp}^{2}}^{\mathrm{adj}}\right) l_{\|+}^{(\mathrm{F})}\left(\tau, 0,-\mathrm{i} \tilde{\mu}_{\tau}\right) \\
& +64 v_{4}\left(-\frac{3}{2} \lambda_{(\sigma-\pi)}^{2}-6 \lambda_{(S+P)_{-}} \lambda_{(\sigma-\pi)}-3 \lambda_{(S+P)_{-}^{\mathrm{adj}}} \lambda_{(\sigma-\pi)}+\frac{3}{N_{c}} \lambda_{(S+P)_{-}^{\mathrm{adj}}} \lambda_{(\sigma-\pi)}-6 \lambda_{(S+P)_{-}}^{2}-\frac{3}{2} \lambda_{(S+P)_{-}^{\mathrm{adj}}}^{2}-\frac{1}{2} \lambda_{(V-A)_{\|}}\right. \\
& -\frac{3}{2} \lambda_{(V-A)_{\perp}}^{2}-\frac{3}{8} \lambda_{(V-A)_{\perp}^{2}}^{\mathrm{adj}}-6 \lambda_{(S+P)_{-}} \lambda_{(S+P)_{-}}{ }_{-}-\frac{3}{2} \lambda_{(V-A)_{\perp}} \lambda_{(V-A)_{\perp}^{\mathrm{adj}}}+\frac{3}{N_{c}} \lambda_{(S+P)_{-}^{\mathrm{adj}}}^{2}+\frac{3}{4 N_{c}} \lambda_{(V-A)_{\perp}^{2}}^{\mathrm{adj}}+\frac{6}{N_{c}} \lambda_{(S+P)_{-}} \lambda_{(S+P)_{-}^{\mathrm{adj}}} \\
& \left.+\frac{3}{2 N_{c}} \lambda_{(V-A) \perp} \lambda_{(V-A)_{\perp}^{\mathrm{adj}}}-\frac{3}{2 N_{c}^{2}} \lambda_{(S+P)_{-}^{2}}^{\mathrm{adj}}-\frac{3}{8 N_{c}^{2}} \lambda_{(V-A)_{\perp}^{2}}^{\mathrm{adj}}\right) l_{\| \pm}^{(\mathrm{F})}\left(\tau, 0,-\mathrm{i} \tilde{\mu}_{\tau}\right)
\end{aligned}
$$




$$
\begin{aligned}
& +64 v_{4}\left(\frac{1}{2} \lambda_{(\sigma-\pi)}^{2}-2 \lambda_{(V+A)} \lambda_{(\sigma-\pi)}-\lambda_{(V+A)_{\|}^{\mathrm{adj}}} \lambda_{(\sigma-\pi)}+\frac{1}{N_{c}} \lambda_{(V+A)_{\|}^{\mathrm{adj}}} \lambda_{(\sigma-\pi)}-\lambda_{(V-A)_{\|}}^{2}-\lambda_{(V-A)_{\perp}}^{2}\right. \\
& +\frac{1}{2} \lambda_{(V+A)_{\|}^{2}}^{\text {adj }}+\lambda_{\mathrm{csc}} \lambda_{(V-A)_{\|}}+4 \lambda_{(V-A)_{\|}} \lambda_{(V-A)_{\perp}}+\lambda_{\mathrm{csc}} \lambda_{(V-A)_{\perp}^{\mathrm{adj}}}+\frac{1}{2} \lambda_{(V-A)_{\|}} \lambda_{(V-A)_{\perp}^{\mathrm{adj}}} \\
& -\lambda_{(V-A)_{\perp}} \lambda_{(V-A)_{\perp}^{\mathrm{adj}}}+2 N_{c} \lambda_{(V-A)_{\|}}^{2}-\frac{N_{c}}{4} \lambda_{(V-A)_{\perp}^{\mathrm{adj}}}^{2}+2 N_{c} \lambda_{(V+A)_{\|}}^{2}-N_{c} \lambda_{\mathrm{csc}} \lambda_{(V-A)_{\|}} \\
& -N_{c} \lambda_{\mathrm{csc}} \lambda_{(V-A)_{\perp}^{\mathrm{adj}}}+\frac{3}{2} N_{c} \lambda_{(V-A)_{\|}} \lambda_{(V-A)_{\perp}^{\mathrm{adj}}}+\frac{1}{2 N_{c}} \lambda_{(V-A)_{\perp}^{\mathrm{adj}}}^{2}-\frac{1}{2 N_{c}} \lambda_{(V+A)_{\|}^{\mathrm{adj}}}^{2} \\
& \left.-\frac{2}{N_{c}} \lambda_{(V-A)_{\|}} \lambda_{(V-A)_{\perp}^{\mathrm{adj}}}+\frac{1}{N_{c}} \lambda_{(V-A)_{\perp}} \lambda_{(V-A)_{\perp}^{\mathrm{adj}}}-\frac{1}{4 N_{c}^{2}} \lambda_{(V-A)_{\perp}^{\mathrm{adj}}}\right) l_{\perp+}^{(\mathrm{F})}\left(\tau, 0,-\mathrm{i} \tilde{\mu}_{\tau}\right) \\
& +64 v_{4}\left(\frac{1}{2} \lambda_{(\sigma-\pi)}^{2}+2 \lambda_{(S+P)_{-}} \lambda_{(\sigma-\pi)}+\lambda_{(S+P)_{-}^{\mathrm{adj}}} \lambda_{(\sigma-\pi)}-\frac{1}{N_{c}} \lambda_{(S+P)_{-}^{\mathrm{adj}}} \lambda_{(\sigma-\pi)}+2 \lambda_{(S+P)_{-}}^{2}+\frac{1}{2} \lambda_{(S+P)_{-}^{\mathrm{adj}}}^{2}\right. \\
& -\lambda_{(V-A)_{\perp}}^{2}-\frac{1}{4} \lambda_{(V-A)_{\perp}^{\mathrm{adj}}}^{2}+2 \lambda_{(S+P)_{-}} \lambda_{(S+P)_{-}^{\mathrm{adj}}}-\lambda_{(V-A)_{\|}} \lambda_{(V-A)_{\perp}}-\frac{1}{2} \lambda_{(V-A)_{\|}} \lambda_{(V-A)_{\perp}^{\mathrm{adj}}} \\
& -\lambda_{(V-A)_{\perp}} \lambda_{(V-A)_{\perp}^{\mathrm{adj}}}-\frac{1}{N_{c}} \lambda_{(S+P)_{-}^{\mathrm{adj}}}^{2}+\frac{1}{2 N_{c}} \lambda_{(V-A)_{\perp}^{\mathrm{adj}}}^{2}-\frac{2}{N_{c}} \lambda_{(S+P)_{-}} \lambda_{(S+P)_{-}^{\mathrm{adj}}} \\
& +\frac{1}{2 N_{c}} \lambda_{(V-A)} \lambda_{(V-A)_{\perp}^{\mathrm{adj}}}+\frac{1}{N_{c}} \lambda_{(V-A)_{\perp}} \lambda_{(V-A)_{\perp}^{\mathrm{adj}}}+\frac{1}{2 N_{c}^{2}} \lambda_{(S+P)_{-}^{\mathrm{adj}}}^{2}-\frac{1}{4 N_{c}^{2}} \lambda_{(V-A)_{\perp}^{\mathrm{adj}}}^{2} l_{\perp \pm}^{\mathrm{F})}\left(\tau, 0,-\mathrm{i} \tilde{\mu}_{\tau}\right), \\
& \partial_{t} \lambda_{(V-A)_{\perp}}=2 \lambda_{(V-A)_{\perp}}+64 v_{4}\left(\frac{N_{c}}{2} \lambda_{\mathrm{csc}}^{2}+\frac{1}{N_{c}} \lambda_{\mathrm{csc}}^{2}-\frac{3}{2} \lambda_{\mathrm{csc}}^{2}+\lambda_{(V-A)_{\perp}} \lambda_{\mathrm{csc}}-\frac{3}{2} \lambda_{(V-A)_{\perp}^{\mathrm{adj}}} \lambda_{\mathrm{csc}}-N_{c} \lambda_{(V-A)_{\perp}} \lambda_{\mathrm{csc}}\right. \\
& +\frac{N_{c}}{2} \lambda_{(V-A)_{\perp}^{\mathrm{adj}}} \lambda_{\mathrm{csc}}+\frac{1}{N_{c}} \lambda_{(V-A)_{\perp}^{\mathrm{adj}}} \lambda_{\mathrm{csc}}-\frac{1}{2} \lambda_{(\sigma-\pi)}^{2}-\frac{5}{8} \lambda_{(V-A)_{\perp}^{\mathrm{adj}}}-\frac{1}{2} \lambda_{(V+A)_{\|}^{2}}^{\mathrm{adj}}+2 \lambda_{(V-A)_{\|}} \lambda_{(V-A)_{\perp}} \\
& +\frac{3}{2} \lambda_{(V-A)_{\perp}} \lambda_{(V-A)_{\perp}^{\mathrm{adj}}}+\lambda_{(\sigma-\pi)} \lambda_{(V+A)_{\|}^{\mathrm{adj}}}-2 \lambda_{(\sigma-\pi)} \lambda_{(V+A)_{\perp}}+2 N_{c} \lambda_{(V-A)_{\perp}}^{2}+\frac{3}{8} N_{c} \lambda_{(V-A)_{\perp}^{\mathrm{adj}}}^{2} \\
& +2 N_{c} \lambda_{(V+A)_{\perp}}^{2}+\frac{N_{c}}{2} \lambda_{(V-A)_{\perp}} \lambda_{(V-A)_{\perp}^{\mathrm{adj}}}+\frac{1}{N_{c}} \lambda_{(\sigma-\pi)}^{2}-\frac{3}{4 N_{c}} \lambda_{(V-A)_{\perp}^{\mathrm{adj}}}^{2}+\frac{1}{2 N_{c}} \lambda_{(V+A)_{\|}^{\mathrm{adj}}}^{2} \\
& \left.-\frac{2}{N_{c}} \lambda_{(V-A)_{\perp}} \lambda_{(V-A)_{\perp}^{\mathrm{adj}}}-\frac{1}{N_{c}} \lambda_{(\sigma-\pi)} \lambda_{(V+A)_{\|}^{\mathrm{adj}}}+\frac{1}{N_{c}^{2}} \lambda_{(V-A)_{\perp}^{\mathrm{adj}}}^{2}\right) l_{\|+}^{(\mathrm{F})}\left(\tau, 0,-\mathrm{i} \tilde{\mu}_{\tau}\right) \\
& +64 v_{4}\left(\frac{1}{N_{c}} \lambda_{(\sigma-\pi)}^{2}-\frac{1}{2} \lambda_{(\sigma-\pi)}^{2}-2 \lambda_{(S+P)_{-}} \lambda_{(\sigma-\pi)}-\lambda_{(S+P)_{-}} \lambda_{(\sigma-\pi)}+\frac{4}{N_{c}} \lambda_{(S+P)_{-}} \lambda_{(\sigma-\pi)}+\frac{3}{N_{c}} \lambda_{(S+P)_{-}^{\mathrm{adj}}} \lambda_{(\sigma-\pi)}\right. \\
& -\frac{2}{N_{c}^{2}} \lambda_{(S+P)_{-}^{\mathrm{adj}}} \lambda_{(\sigma-\pi)}-2 \lambda_{(S+P)_{-}}^{2}-\frac{1}{2} \lambda_{(S+P)_{-}^{\mathrm{adj}}}^{2}-\lambda_{(V-A)_{\perp}}^{2}-\frac{1}{4} \lambda_{(V-A)_{\perp}^{\mathrm{adj}}}^{2}-2 \lambda_{(S+P)_{-}} \lambda_{(S+P)_{-}^{\mathrm{adj}}}-\lambda_{(V-A)_{\|}} \lambda_{(V-A)_{\perp}} \\
& -\frac{3}{2} \lambda_{(V-A)_{\perp}} \lambda_{(V-A)_{\perp}^{\mathrm{adj}}}+\frac{4}{N_{c}} \lambda_{(S+P)_{-}}^{2}+\frac{2}{N_{c}} \lambda_{(S+P)_{-}^{\mathrm{adj}}}^{2}+\frac{3}{4 N_{c}} \lambda_{(V-A)_{\perp}^{\mathrm{adj}}}^{2}+\frac{6}{N_{c}} \lambda_{(S+P)_{-}} \lambda_{(S+P)_{-}^{\mathrm{adj}}}+\frac{3}{2 N_{c}} \lambda_{(V-A)_{\perp}} \lambda_{(V-A)_{\perp}^{\mathrm{adj}}} \\
& \left.-\frac{5}{2 N_{c}^{2}} \lambda_{(S+P)_{-}^{\mathrm{adj}}}^{2}-\frac{1}{2 N_{c}^{2}} \lambda_{(V-A)_{\perp}^{\mathrm{adj}}}^{2}-\frac{4}{N_{c}^{2}} \lambda_{(S+P)_{-}} \lambda_{(S+P)_{-}^{\mathrm{adj}}}+\frac{1}{N_{c}^{3}} \lambda_{(S+P)_{-}^{\mathrm{adj}}}^{2}\right) l_{\| \pm}^{(\mathrm{F})}\left(\tau, 0,-\mathrm{i} \tilde{\mu}_{\tau}\right) \\
& +64 v_{4}\left(-\frac{1}{3 N_{c}} \lambda_{\mathrm{csc}}^{2}+\frac{1}{3} \lambda_{\mathrm{csc}}^{2}+\frac{1}{3} \lambda_{(V-A)} \lambda_{\mathrm{csc}}+\lambda_{(V-A)_{\perp}^{\mathrm{adj}}} \lambda_{\mathrm{csc}}-\frac{N_{c}}{3} \lambda_{(V-A)_{\perp}} \lambda_{\mathrm{csc}}\right. \\
& -\frac{N_{c}}{3} \lambda_{(V-A)_{\perp}^{\mathrm{adj}}} \lambda_{\mathrm{csc}}-\frac{2}{3 N_{c}} \lambda_{(V-A)_{\perp}^{\mathrm{adj}}} \lambda_{\mathrm{csc}}+\frac{1}{2} \lambda_{(\sigma-\pi)}^{2}+\frac{1}{6} \lambda_{(V-A)_{\|}}^{2}+\frac{3}{2} \lambda_{(V-A)_{\perp}}^{2}+\frac{13}{24} \lambda_{(V-A)_{\perp}^{\mathrm{adj}}}^{2} \\
& +\frac{1}{2} \lambda_{(V+A)_{\|}^{\mathrm{adj}}}^{2}-\frac{1}{3} \lambda_{(V-A)_{\|}} \lambda_{(V-A)_{\perp}}+\frac{1}{2} \lambda_{(V-A)_{\|}} \lambda_{(V-A)_{\perp}^{\mathrm{adj}}}-\lambda_{(V-A)_{\perp}} \lambda_{(V-A)_{\perp}^{\mathrm{adj}}}-\lambda_{(\sigma-\pi)} \lambda_{(V+A)_{\|}^{\mathrm{adj}}} \\
& -\frac{2}{3} \lambda_{(\sigma-\pi)} \lambda_{(V+A)_{\perp}}+\frac{2}{3} N_{c} \lambda_{(V-A)_{\perp}}^{2}-\frac{N_{c}}{4} \lambda_{(V-A)_{\perp}^{2}}^{\text {adj }}+\frac{2}{3} N_{c} \lambda_{(V+A)_{\perp}}^{2}+\frac{N_{c}}{6} \lambda_{(V-A)_{\perp}} \lambda_{(V-A)_{\perp}^{\mathrm{adj}}}
\end{aligned}
$$




$$
\begin{aligned}
& -\frac{1}{3 N_{c}} \lambda_{(\sigma-\pi)}^{2}+\frac{1}{2 N_{c}} \lambda_{(V-A)_{\perp}^{\mathrm{adj}}}^{2}-\frac{1}{2 N_{c}} \lambda_{(V+A)_{\|}^{2}}^{\mathrm{adj}}-\frac{1}{2 N_{c}} \lambda_{(V-A)} \lambda_{(V-A)_{\perp}^{\mathrm{adj}}} \\
& \left.+\frac{5}{6 N_{c}} \lambda_{(V-A) \perp} \lambda_{(V-A)_{\perp}^{\mathrm{adj}}}+\frac{1}{N_{c}} \lambda_{(\sigma-\pi)} \lambda_{(V+A)_{\|}^{\mathrm{adj}}}-\frac{19}{24 N_{c}^{2}} \lambda_{(V-A)_{\perp}^{2}}^{\mathrm{adj}}\right) l_{\perp+}^{(\mathrm{F})}\left(\tau, 0,-\mathrm{i} \tilde{\mu}_{\tau}\right) \\
& +64 v_{4}\left(-\frac{1}{3 N_{c}} \lambda_{(\sigma-\pi)}^{2}+\frac{1}{6} \lambda_{(\sigma-\pi)}^{2}+\frac{2}{3} \lambda_{(S+P)_{-}} \lambda_{(\sigma-\pi)}+\frac{1}{3} \lambda_{(S+P)_{-}^{\mathrm{adj}}} \lambda_{(\sigma-\pi)}-\frac{4}{3 N_{c}} \lambda_{(S+P)_{-}} \lambda_{(\sigma-\pi)}\right. \\
& -\frac{1}{N_{c}} \lambda_{(S+P)_{-}^{\mathrm{adj}}} \lambda_{(\sigma-\pi)}+\frac{2}{3 N_{c}^{2}} \lambda_{(S+P)_{-}^{\mathrm{adj}}} \lambda_{(\sigma-\pi)}+\frac{2}{3} \lambda_{(S+P)_{-}}^{2}+\frac{1}{6} \lambda_{(S+P)_{-}^{2}}^{2}-\frac{1}{6} \lambda_{(V-A)_{\|}}^{2}-\frac{7}{6} \lambda_{(V-A)_{\perp}}^{2} \\
& -\frac{7}{24} \lambda_{(V-A)_{\perp}^{\mathrm{adj}}}^{2}+\frac{2}{3} \lambda_{(S+P)_{-}} \lambda_{(S+P)_{-}^{\mathrm{adj}}}-\frac{2}{3} \lambda_{(V-A)_{\|}} \lambda_{(V-A)_{\perp}}-\frac{1}{2} \lambda_{(V-A)_{\|}} \lambda_{(V-A)_{\perp}^{\mathrm{adj}}} \\
& -\lambda_{(V-A)_{\perp}} \lambda_{(V-A)_{\perp}^{\mathrm{adj}}}-\frac{4}{3 N_{c}} \lambda_{(S+P)_{-}}^{2}-\frac{2}{3 N_{c}} \lambda_{(S+P)_{-}^{\mathrm{adj}}}^{2}+\frac{1}{2 N_{c}} \lambda_{(V-A)_{\perp}^{\mathrm{adj}}}^{2}-\frac{2}{N_{c}} \lambda_{(S+P)_{-}} \lambda_{(S+P)_{-}^{\mathrm{ajj}}} \\
& +\frac{1}{2 N_{c}} \lambda_{(V-A)} \lambda_{(V-A)_{\perp}^{\mathrm{adj}}}+\frac{1}{N_{c}} \lambda_{(V-A) \perp} \lambda_{(V-A)_{\perp}^{\mathrm{adj}}}+\frac{5}{6 N_{c}^{2}} \lambda_{(S+P)_{\perp}^{2}}^{2}-\frac{5}{24 N_{c}^{2}} \lambda_{(V-A)_{\perp}^{2}}^{\text {adj }} \\
& \left.+\frac{4}{3 N_{c}^{2}} \lambda_{(S+P)_{-}} \lambda_{(S+P)_{-}^{\mathrm{adj}}}-\frac{1}{3 N_{c}^{3}} \lambda_{(S+P)_{-}^{\mathrm{adj}}}^{2}\right) l_{\perp \pm}^{(\mathrm{F})}\left(\tau, 0,-\mathrm{i} \tilde{\mu}_{\tau}\right), \\
& \partial_{t} \lambda_{(V+A)_{\|}^{\mathrm{adj}}}=2 \lambda_{(V+A)_{\|}^{\mathrm{adj}}}+64 v_{4}\left(2 N_{c} \lambda_{\mathrm{csc}} \lambda_{(S+P)_{-}^{\mathrm{adj}}}-\frac{2}{N_{c}} \lambda_{\mathrm{csc}} \lambda_{(S+P)_{-}^{\mathrm{adj}}}+N_{c} \lambda_{\mathrm{csc}}^{2}-\frac{2}{N_{c}} \lambda_{(\sigma-\pi)} \lambda_{(V-A)_{\perp}^{\mathrm{adj}}}\right.
\end{aligned}
$$

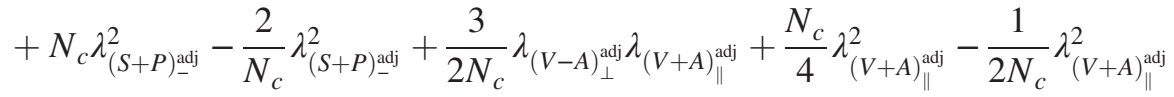

$$
\begin{aligned}
& +4 \lambda_{\mathrm{csc}} \lambda_{(\sigma-\pi)}+4 \lambda_{\mathrm{csc}} \lambda_{(S+P)_{-}}-2 \lambda_{\mathrm{csc}} \lambda_{(S+P)_{-}^{\mathrm{adj}}}-\lambda_{\mathrm{csc}} \lambda_{(V+A)_{\|}^{\mathrm{adj}}}-2 \lambda_{\mathrm{csc}}^{2}+2 \lambda_{(\sigma-\pi)} \lambda_{(S+P)_{-}^{\mathrm{adj}}} \\
& +4 \lambda_{(\sigma-\pi)} \lambda_{(V-A)_{\perp}}+2 \lambda_{(\sigma-\pi)} \lambda_{(V-A)_{\perp}^{\mathrm{adj}}}+4 \lambda_{(S+P)_{-}} \lambda_{(S+P)_{-}^{\mathrm{adj}}}+\lambda_{(V-A)_{\|}} \lambda_{(V+A)_{\|}^{\mathrm{adj}}} \\
& \left.-3 \lambda_{(V-A) \perp} \lambda_{(V+A))_{\|}^{\text {adj }}}+\lambda_{(V+A) \|} \lambda_{(V+A)_{\|}^{\mathrm{adj}}}-\lambda_{(V+A)_{\|}^{\mathrm{adj}}} \lambda_{(V+A)_{\perp}}\right) l_{\|+}^{\mathrm{F})}\left(\tau, 0,-\mathrm{i} \tilde{\mu}_{\tau}\right) \\
& +64 v_{4}\left(\frac{1}{2 N_{c}} \lambda_{(V+A)_{\|}^{\mathrm{adj}}}^{2}+4 \lambda_{(\sigma-\pi)} \lambda_{(V+A)_{\perp}}-\lambda_{(V+A) \|} \lambda_{(V+A)_{\|}^{\mathrm{adj}}}+\lambda_{(V+A)_{\|}^{\mathrm{adj}}} \lambda_{(V+A)_{\perp}}\right) l_{\| \pm}^{(\mathrm{F})}\left(\tau, 0,-\mathrm{i} \tilde{\mu}_{\tau}\right) \\
& +64 v_{4}\left(-\frac{2}{3} N_{c} \lambda_{\mathrm{csc}} \lambda_{(S+P)_{-}^{\mathrm{adj}}}+\frac{2}{3 N_{c}} \lambda_{\mathrm{csc}} \lambda_{(S+P)_{-}^{\mathrm{adj}}}-\frac{N_{c}}{3} \lambda_{\mathrm{csc}}^{2}+\frac{4}{3 N_{c}} \lambda_{(\sigma-\pi)} \lambda_{(V-A)_{\perp}^{\mathrm{adj}}}\right. \\
& -\frac{N_{c}}{3} \lambda_{(S+P)_{-}^{\mathrm{adj}}}^{2}+\frac{2}{3 N_{c}} \lambda_{(S+P)_{-}^{\mathrm{adj}}}^{2}-\frac{3}{2 N_{c}} \lambda_{(V-A)_{\perp}^{\mathrm{adj}}} \lambda_{(V+A)_{\|}^{\mathrm{adj}}}-\frac{N_{c}}{12} \lambda_{(V+A)_{\|}^{2}}^{\mathrm{adj}}+\frac{1}{6 N_{c}} \lambda_{(V+A)_{\|}^{\mathrm{adj}}}^{2} \\
& \left.-\frac{4}{3} \lambda_{\mathrm{csc}} \lambda_{(\sigma-\pi)}-\frac{4}{3} \lambda_{\mathrm{csc}} \lambda_{(S+P)_{-}}+\frac{2}{3} \lambda_{\mathrm{csc}} \lambda_{(S+P)_{-}^{\mathrm{adj}}}+\lambda_{\mathrm{csc}} \lambda_{(V+A)_{\|}^{\mathrm{adj}}}+\frac{2}{3} \lambda_{\mathrm{csc}}^{2}-\frac{2}{3} \lambda_{(\sigma-\pi)} \lambda_{(S+P)}\right)_{-}^{\mathrm{adj}} \\
& +\frac{4}{3} \lambda_{(\sigma-\pi)} \lambda_{(V-A)_{\|}}-\frac{8}{3} \lambda_{(\sigma-\pi)} \lambda_{(V-A)_{\perp}}+\frac{2}{3} \lambda_{(\sigma-\pi)} \lambda_{(V-A)_{\perp}^{\mathrm{adj}}}-\frac{4}{3} \lambda_{(S+P)_{-}} \lambda_{(S+P)_{-}^{\mathrm{adj}}}-\lambda_{(V-A)_{\|}} \lambda_{(V+A)_{\|}^{\mathrm{adj}}} \\
& \left.+3 \lambda_{(V-A)_{\perp}} \lambda_{(V+A)_{\|}^{\mathrm{adj}}}-\frac{1}{3} \lambda_{(V+A)_{\|}} \lambda_{(V+A)_{\|}^{\mathrm{adj}}}+\frac{1}{3} \lambda_{(V+A)_{\|}^{\mathrm{adj}}} \lambda_{(V+A)_{\perp}}\right) l_{\perp+}^{(\mathrm{F})}\left(\tau, 0,-\mathrm{i} \tilde{\mu}_{\tau}\right) \\
& +64 v_{4}\left(-\frac{2}{3 N_{c}} \lambda_{(\sigma-\pi)} \lambda_{(V+A)}{ }_{\|}^{\mathrm{adj}}-\frac{1}{6 N_{c}} \lambda_{(V+A)_{\|}^{\mathrm{adj}}}+\frac{4}{3} \lambda_{(\sigma-\pi)} \lambda_{(V+A) \|}-\frac{8}{3} \lambda_{(\sigma-\pi)} \lambda_{(V+A)_{\perp}}\right. \\
& \left.+\frac{1}{3} \lambda_{(V+A)_{\|}} \lambda_{(V+A)_{\|}^{\mathrm{adj}}}-\frac{5}{3} \lambda_{(V+A)_{\|}^{\mathrm{ajj}}} \lambda_{(V+A)_{\perp}}\right) l_{\perp \pm}^{\mathrm{F})}\left(\tau, 0,-\mathrm{i} \tilde{\mu}_{\tau}\right),
\end{aligned}
$$




$$
\begin{aligned}
& \partial_{t} \lambda_{(V-A)_{\perp}^{\mathrm{adj}}}=2 \lambda_{(V-A)_{\perp}^{\mathrm{adj}}}+64 v_{4}\left(-2 N_{c} \lambda_{\mathrm{csc}} \lambda_{(V-A)_{\perp}^{\mathrm{adj}}}-N_{c} \lambda_{\mathrm{csc}}^{2}-\frac{5}{4} N_{c} \lambda_{(V-A)_{\perp}^{\mathrm{adj}}}^{2}+\frac{2}{N_{c}} \lambda_{(V-A)_{\perp}^{\mathrm{adj}}}^{2}\right. \\
& +3 \lambda_{\mathrm{csc}} \lambda_{(V-A)_{\perp}^{\mathrm{adj}}}+2 \lambda_{\mathrm{csc}}^{2}-2 \lambda_{(\sigma-\pi)} \lambda_{(V+A)_{\|}^{\mathrm{adj}}}+2 \lambda_{(\sigma-\pi)}^{2}+2 \lambda_{(V-A)} \lambda_{(V-A)_{\perp}^{\mathrm{adj}}} \\
& \left.-4 \lambda_{(V-A)_{\perp}} \lambda_{(V-A)_{\perp}^{\mathrm{adj}}}+\lambda_{(V-A)_{\perp}^{\mathrm{adj}}}^{2}+\lambda_{(V+A)_{\|}^{\mathrm{adj}}}^{2}\right) l_{\|+}^{(\mathrm{F})}\left(\tau, 0,-\mathrm{i} \tilde{\mu}_{\tau}\right) \\
& +64 v_{4}\left(-\frac{4}{N_{c}} \lambda_{(\sigma-\pi)} \lambda_{(S+P)_{-}^{\mathrm{adj}}}-\frac{8}{N_{c}} \lambda_{(S+P)_{-}} \lambda_{(S+P)_{-}^{\mathrm{adj}}}-\frac{4}{N_{c}} \lambda_{(S+P)_{-}^{2}}^{\text {adj }}+\frac{2}{N_{c}^{2}} \lambda_{(S+P)_{-}^{\mathrm{adj}}}^{2}\right. \\
& -\frac{1}{2 N_{c}} \lambda_{(V-A)_{\perp}^{\mathrm{adj}}}^{2}+8 \lambda_{(\sigma-\pi)} \lambda_{(S+P)_{-}}+4 \lambda_{(\sigma-\pi)} \lambda_{(S+P)_{-}^{\mathrm{adj}}}+2 \lambda_{(\sigma-\pi)}^{2}+8 \lambda_{(S+P)_{-}} \lambda_{(S+P)_{-}^{\mathrm{adj}}} \\
& \left.+8 \lambda_{(S+P)_{-}}^{2}+2 \lambda_{(S+P)_{-}^{\mathrm{adj}}}^{2}-\lambda_{(V-A)_{\|}} \lambda_{(V-A)_{\perp}^{\mathrm{adj}}}+\lambda_{(V-A)_{\perp}} \lambda_{(V-A)_{\perp}^{\mathrm{adj}}}\right) l_{\| \pm}^{(\mathrm{F})}\left(\tau, 0,-\mathrm{i} \tilde{\mu}_{\tau}\right) \\
& +64 v_{4}\left(\frac{4}{3} N_{c} \lambda_{\mathrm{csc}} \lambda_{(V-A)_{\perp}^{\mathrm{adj}}}+\frac{N_{c}}{3} \lambda_{\mathrm{csc}}^{2}+\frac{13}{12} N_{c} \lambda_{(V-A)_{\perp}^{\mathrm{adj}}}^{2}-\frac{7}{3 N_{c}} \lambda_{(V-A)_{\perp}^{\mathrm{adj}}}^{2}-\lambda_{\mathrm{csc}} \lambda_{(V-A)_{\perp}^{\mathrm{adj}}}\right. \\
& -\frac{2}{3} \lambda_{\mathrm{csc}}^{2}+2 \lambda_{(\sigma-\pi)} \lambda_{(V+A)_{\|}^{\mathrm{adj}}}-\frac{2}{3} \lambda_{(\sigma-\pi)}^{2}-\frac{4}{3} \lambda_{(V-A)} \lambda_{(V-A)_{\perp}^{\mathrm{adj}}}+\frac{14}{3} \lambda_{(V-A)_{\perp}} \lambda_{(V-A)_{\perp}^{\mathrm{adj}}}+\frac{1}{3} \lambda_{(V-A)_{\perp}^{\mathrm{adj}}}^{2} \lambda_{(V+A)_{\|}^{\mathrm{adj}}}^{2} l_{\perp+}^{(\mathrm{F})}\left(\tau, 0,-\mathrm{i} \tilde{\mu}_{\tau}\right) \\
& +64 v_{4}\left(\frac{4}{3 N_{c}} \lambda_{(\sigma-\pi)} \lambda_{(S+P)_{-}^{\mathrm{adj}}}+\frac{8}{3 N_{c}} \lambda_{(S+P)_{-}} \lambda_{(S+P)_{-}^{\mathrm{adj}}}+\frac{4}{3 N_{c}} \lambda_{(S+P)_{-}^{\mathrm{adj}}}^{2}-\frac{2}{3 N_{c}^{2}} \lambda_{(S+P)_{-}^{\mathrm{adj}}}^{2}\right. \\
& +\frac{1}{6 N_{c}} \lambda_{(V-A)_{\perp}^{\mathrm{adj}}}^{2}-\frac{8}{3} \lambda_{(\sigma-\pi)} \lambda_{(S+P)_{-}}-\frac{4}{3} \lambda_{(\sigma-\pi)} \lambda_{(S+P)_{-}^{\mathrm{adj}}}-\frac{2}{3} \lambda_{(\sigma-\pi)}^{2}-\frac{8}{3} \lambda_{(S+P)_{-}} \lambda_{(S+P)_{-}^{\mathrm{adj}}} \\
& \left.-\frac{8}{3} \lambda_{(S+P)_{-}}^{2}-\frac{2}{3} \lambda_{(S+P)_{-}^{\mathrm{adj}}}^{2}+\frac{1}{3} \lambda_{(V-A)_{\|}} \lambda_{(V-A)_{\perp}^{\mathrm{adj}}}-\frac{1}{3} \lambda_{(V-A)_{\perp}} \lambda_{(V-A)_{\perp}^{\mathrm{adj}}}\right) l_{\perp \pm}^{(\mathrm{F})}\left(\tau, 0,-\mathrm{i} \tilde{\mu}_{\tau}\right) .
\end{aligned}
$$

[1] A. L. Watts, N. Andersson, D. Chakrabarty, M. Feroci, K. Hebeler, G. Israel, F. K. Lamb, M. C. Miller, S. Morsink, F. Özel, A. Patruno, J. Poutanen, D. Psaltis, A. Schwenk, A. W. Steiner, L. Stella, L. Tolos, and M. van der Klis, Rev. Mod. Phys. 88, 021001 (2016).

[2] Y. Nambu and G. Jona-Lasinio, Phys. Rev. 122, 345 (1961); 124, 246 (1961).

[3] T. Hatsuda and T. Kunihiro, Phys. Rep. 247, 221 (1994).

[4] S. P. Klevansky, Rev. Mod. Phys. 64, 649 (1992).

[5] M. Buballa, Phys. Rep. 407, 205 (2005).

[6] K. Fukushima, J. Phys. G 39, 013101 (2012).

[7] J. O. Andersen, W. R. Naylor, and A. Tranberg, Rev. Mod. Phys. 88, 025001 (2016).

[8] J. Braun, J. Phys. G 39, 033001 (2012).

[9] J. Braun and T. K. Herbst, arXiv:1205.0779.

[10] J. Braun, M. Leonhardt, and M. Pospiech, Phys. Rev. D 96, 076003 (2017).

[11] P. Springer, J. Braun, S. Rechenberger, and F. Rennecke, Eur. Phys. J. Web Conf. 137, 03022 (2017).

[12] D. Bailin and A. Love, Phys. Rep. 107, 325 (1984).
[13] M. G. Alford, A. Schmitt, K. Rajagopal, and T. Schäfer, Rev. Mod. Phys. 80, 1455 (2008).

[14] R. Anglani, R. Casalbuoni, M. Ciminale, N. Ippolito, R. Gatto, M. Mannarelli, and M. Ruggieri, Rev. Mod. Phys. 86, 509 (2014).

[15] J. J. Rusnak and R. J. Furnstahl, Z. Phys. A 352, 345 (1995).

[16] S. Pokorski, Gauge Field Theories (Cambridge University Press, Cambridge, England, 1987).

[17] G. 't Hooft, Phys. Rev. Lett. 37, 8 (1976).

[18] G.'t Hooft, Phys. Rev. D 14, 3432 (1976); 18, 2199(E) (1978).

[19] M. Kobayashi and T. Maskawa, Prog. Theor. Phys. 44, 1422 (1970); M. Kobayashi, H. Kondo, and T. Maskawa, Prog. Theor. Phys. 45, 1955 (1971).

[20] T. P. Cheng and L. F. Li, Gauge Theory of Elementary Particle Physics (Oxford University Press, USA1984).

[21] M. A. Shifman, A. I. Vainshtein, and V. I. Zakharov, Nucl. Phys. B163, 46 (1980).

[22] E. V. Shuryak, Nucl. Phys. B203, 93 (1982).

[23] T. Schäfer and E. V. Shuryak, Rev. Mod. Phys. 70, 323 (1998).

[24] J. M. Pawlowski, Phys. Rev. D 58, 045011 (1998). 
[25] M. G. Alford, K. Rajagopal, and F. Wilczek, Phys. Lett. B 422, 247 (1998).

[26] R. Rapp, T. Schäferx, E. V. Shuryak, and M. Velkovsky, Phys. Rev. Lett. 81, 53 (1998).

[27] M. Buballa, Prog. Part. Nucl. Phys. 61, 66 (2008).

[28] I. A. Shovkovy, Found. Phys. 35, 1309 (2005).

[29] T. D. Cohen, Phys. Rev. Lett. 91, 222001 (2003).

[30] G. Markó, U. Reinosa, and Z. Szep, Phys. Rev. D 90, 125021 (2014).

[31] N. Khan, J. M. Pawlowski, F. Rennecke, and M. M. Scherer, arXiv:1512.03673; W.-j. Fu and J. M. Pawlowski, Phys. Rev. D 92, 116006 (2015).

[32] J. Hubbard, Phys. Rev. Lett. 3, 77 (1959); R. Stratonovich, Doklady / Akademiia nauk Azerbaidzhanskoi SSR 115, 1097 (1957).

[33] H. Gies and J. Jaeckel, Eur. Phys. J. C 46, 433 (2006); J. Braun and H. Gies, Phys. Lett. B 645, 53 (2007); J. High Energy Phys. 06 (2006) 024; J. Braun, H. Gies, L. Janssen, and D. Roscher, Phys. Rev. D 90, 036002 (2014).

[34] C. Wetterich, Phys. Lett. B 301, 90 (1993).

[35] J. Braun, Ph.D. thesis, Heidelberg University, 2006.

[36] M. Mitter, J. M. Pawlowski, and N. Strodthoff, Phys. Rev. D 91, 054035 (2015).

[37] A. K. Cyrol, M. Mitter, J. M. Pawlowski, and N. Strodthoff, Phys. Rev. D 97, 054006 (2018).

[38] J. Braun, L. Fister, J. M. Pawlowski, and F. Rennecke, Phys. Rev. D 94, 034016 (2016).

[39] D. F. Litim, Phys. Lett. B 486, 92 (2000); Phys. Rev. D 64, 105007 (2001); Int. J. Mod. Phys. A 16, 2081 (2001).

[40] D. U. Jungnickel and C. Wetterich, Phys. Rev. D 53, 5142 (1996).

[41] J. Berges, D. U. Jungnickel, and C. Wetterich, Phys. Rev. D 59, 034010 (1999).

[42] J. M. Pawlowski, Ann. Phys. (Amsterdam) 322, 2831 (2007).
[43] J. Braun, Eur. Phys. J. C 64, 459 (2009).

[44] J. Braun, Phys. Rev. D 81, 016008 (2010).

[45] M. Asakawa and K. Yazaki, Nucl. Phys. A504, 668 (1989).

[46] B.-J. Schaefer and M. Wagner, Phys. Rev. D 79, 014018 (2009).

[47] T. Hatsuda and T. Kunihiro, Phys. Rev. Lett. 55, 158 (1985).

[48] J. Jaeckel and C. Wetterich, Phys. Rev. D 68, 025020 (2003).

[49] H. Gies, Lect. Notes Phys. 852, 287 (2012).

[50] J. Berges and K. Rajagopal, Nucl. Phys. B538, 215 (1999).

[51] D. T. Son, Phys. Rev. D 59, 094019 (1999); R. D. Pisarski and D. H. Rischke, Phys. Rev. D 61, 074017 (2000); 61, 051501 (2000); T. Schäfer and F. Wilczek, Phys. Rev. D 60, 114033 (1999); W. E. Brown, J. T. Liu, and H.-c. Ren, Phys. Rev. D 61, 114012 (2000); D. K. Hong, V. A. Miransky, I. A. Shovkovy, and L. C. R. Wijewardhana, Phys. Rev. D 61, 056001 (2000); 62, 059903(E) (2000); N. J. Evans, J. Hormuzdiar, S. D. H. Hsu, and M. Schwetz, Nucl. Phys. B581, 391 (2000).

[52] H. Gies and L. Janssen, Phys. Rev. D 82, 085018 (2010).

[53] N. Strodthoff, B.-J. Schaefer, and L. von Smekal, Phys. Rev. D 85, 074007 (2012); K. Kamikado, N. Strodthoff, L. von Smekal, and J. Wambach, Phys. Lett. B 718, 1044 (2013); N. Strodthoff and L. von Smekal, Phys. Lett. B 731, 350 (2014).

[54] F. Rennecke, Phys. Rev. D 92, 076012 (2015).

[55] J. Braun, L. Corell, A. K. Cyrol, W.-j. Fu, M. Leonhardt, M. Mitter, J. M. Pawlowski, M. Pospiech, F. Rennecke, N. Wink (fQCD Collaboration).

[56] F. Gehring, H. Gies, and L. Janssen, Phys. Rev. D 92, 085046 (2015).

[57] M. Q. Huber and J. Braun, Comput. Phys. Commun. 183, 1290 (2012).

[58] A. K. Cyrol, M. Mitter, and N. Strodthoff, Comput. Phys. Commun. 219, 346 (2017). 